\title{
Interventions to Reduce Meat Consumption by Appealing to Animal Welfare: Meta-Analysis and Evidence-Based Recommendations
}

\author{
Maya B. Mathur*1, Jacob Peacock ${ }^{\dagger 2}$, David B. Reichling ${ }^{\ddagger 3}$, Janice Nadler ${ }^{\S 4,5}$, \\ Paul A. Bain ${ }^{\llbracket 6}$, Christopher D. Gardner ${ }^{\| 7}$, and Thomas N. Robinson**8 \\ ${ }^{1}$ Quantitative Sciences Unit, Stanford University \\ ${ }^{2}$ The Humane League Labs \\ ${ }^{3}$ Department of Oral and Maxillofacial Surgery, University of California at San \\ Francisco (ret.) \\ ${ }^{4}$ American Bar Foundation \\ ${ }^{5}$ Pritzker School of Law, Northwestern University \\ ${ }^{6}$ Countway Library of Medicine, Harvard University \\ ${ }^{7}$ Stanford Prevention Research Center, Stanford University \\ ${ }^{8}$ Stanford Solutions Science Lab, Department of Pediatrics, Stanford University
}

Citation: Mathur MB, Peacock J, Reichling DB, Nadler J, Bain PA, Gardner CD, Robinson $\mathrm{TN}$ (in press). Interventions to reduce meat consumption by appealing to animal welfare: Meta-analysis and evidence-based recommendations. Appetite.

${ }^{*}$ Correspondence to: Maya B. Mathur (mmathur@stanford.edu), Quantitative Sciences Unit, 1701 Page Mill Road, Palo Alto, CA, 94304.

†jpeacock@thehumaneleague.org

${ }_{\ddagger}^{\ddagger}$ nervefiber@gmail.com

§nnadler@law.northwestern.edu

I paul_bain@hms.harvard.edu

" cgardner@stanford.edu

**tom.robinson@stanford.edu 


\begin{abstract}
Reducing meat consumption may improve human health, curb environmental damage, and limit the large-scale suffering of animals reared in factory farms. Most attention to reducing consumption has focused on restructuring environments where foods are chosen or on making health or environmental appeals. However, psychological theory suggests that interventions appealing to animal welfare concerns might operate on distinct, potent pathways. We conducted a systematic review and meta-analysis evaluating the effectiveness of these interventions. We searched eight academic databases and extensively searched grey literature. We meta-analyzed 100 studies assessing interventions designed to reduce meat consumption or purchase by mentioning or portraying farm animals, that measured behavioral or self-reported outcomes related to meat consumption, purchase, or related intentions, and that had a control condition. The interventions consistently reduced meat consumption, purchase, or related intentions at least in the short term with meaningfully large effects (meta-analytic mean risk ratio $[R R]=1.22 ; 95 \% \mathrm{CI}$ : $[1.13,1.33])$. We estimated that a large majority of true population effects $(71 \% ; 95 \%$ CI: $[59 \%, 80 \%])$ were stronger than $R R=1.1$ and that few were in the unintended direction. Via meta-regression, we identified some specific characteristics of studies and interventions that were associated with effect size. Risk-of-bias assessments identified both methodological strengths and limitations of this literature; however, results did not differ meaningfully in sensitivity analyses retaining only studies at the lowest risk of bias. Evidence of publication bias was not apparent. In conclusion, animal welfare interventions preliminarily appear effective in these typically short-term studies of primarily self-reported outcomes. Future research should use direct behavioral outcomes that minimize the potential for social desirability bias and are measured over long-term follow-up.
\end{abstract}

Key words: meta-analysis, nutrition, behavior interventions, meat consumption, planetary health 


\section{INTRODUCTION}

Excessive consumption of meat and animal products may be deleterious to human health (with meta-analytic evidence regarding cancer (Larsson and Wolk, 2006, Farvid et al., 2018, Gnagnarella et al., 2018, Crippa et al., 2018), cardiovascular disease (Guasch-Ferré et al., 2019: Cui et al., 2019, Zhang and Zhang, 2018), metabolic disease (Pan et al., 2011, Kim and Je, 2018; Fretts et al., 2015), obesity (Rouhani et al., 2014), stroke (Kim et al., 2017), and all-cause mortality (Wang et al., 2016; Larsson and Orsini, 2013)); promotes the emergence and spread of pandemics and antibiotic-resistant pathogens (Bartlett et al., 2013, Marshall and Levy, 2011, Di Marco et al., 2020); is a major source of greenhouse gas emissions, environmental degradation, and biodiversity loss Sakadevan and Nguyen, 2017, Machovina et al., 2015); and contributes to the preventable suffering and slaughter of approximately 500 to 12,000 animals over the lifetime of each human consuming a diet typical of his or her country (Scherer et al., 2019, Bonnet et al., 2020) 1 Therefore, developing simple, effective interventions to reduce meat consumption could carry widespread societal benefits.

"Nudge" interventions that restructure the physical environment, for example by repositioning meat dishes in cafeterias or making vegetarian options the default, may be effective (Bianchi et al. 2018b Hansen et al., 2019, Garnett et al., 2019), as may direct appeals regarding individual health or the environment (Bianchi et al., 2018a; Jalil et al., 2019). Despite sustained academic interest in developing those types of interventions, much less attention has been paid to the potential effectiveness of appeals related to animal welfare (Bianchi et al., 2018a). However, the emerging literature on the psychology of meat consumption suggests that appeals to animal welfare might operate on distinct and powerful psychological pathways (Rothgerber, 2020), suggesting that these appeals merit assessment as a potentially effective component of interventions to reduce meat consumption. We first provide a theoretical review of this psychological literature.

\footnotetext{
${ }^{a}$ These are the minimum and maximum taken across 37 countries (Scherer et al. 2019).
} 


\subsection{Psychological theory underlying animal welfare interventions}

A number of interventions have used psychologically sophisticated approaches to reducing meat consumption by appealing to or portraying the welfare of animals reared for meat (henceforth "animal welfare interventions"). In general, portraying a desired behavior as aligning with injunctive social norms (what others believe one should do) or descriptive social norms (what others actually do) can effectively shift behaviors, including food choices (Schultz et al., 2007, Higgs, 2015). Many animal welfare interventions have invoked social norms (Reese, 2015; Amiot et al., 2018; Hennessy, 2016, Norris, 2014, Norris and Roberts, 2016; Norris and Hannan, 2019), for example by stating: "You can't help feeling that eliminating meat is becoming unavoidably mainstream, with more and more people choosing to become vegetarians by cutting out red meat, poultry, and seafood from their diets" (Macdonald et al. 2016). Animal welfare interventions have also leveraged the "identifiable victim effect", in which people often experience stronger affective reactions when considering a single, named victim rather than multiple victims or a generic group (Jenni and Loewenstein, 1997). In a classic demonstration of this general effect, subjects made larger real donations to the organization Save the Children after reading about a single named child than when reading about multiple children (Västfjäll et al. 2014). Analogously, many animal welfare interventions describe farm animals with reference to specific, named individuals, such as "Leon" the pig (Bertolaso, 2015) or "Lucy" the chicken (Reese, 2015), and this may indeed be more effective at shifting behavior than providing statistical descriptions of the number of animals affected. Last, many interventions provide concrete implementation suggestions for reducing meat consumption, for example by listing plant-based dishes or recipes for breakfasts, lunches, and dinners (Norris and Hannan, 2019). These suggestions may help individuals to form concrete intentions for how they would like to respond in the future when faced with food options, which can be an effective means of shifting food choices Adriaanse et al., 2011). 
In addition to leveraging these three well-known standard components of effective behavioral interventions, animal welfare interventions have the potential to harness the unique social, moral, and affective psychology underlying meat consumption (Loughnan et al., 2014; Rozin, 1996). For example, ethical concern about factory farming conditions is now a majority stance in several developed countries (Cornish et al. 2016), yet meat consumption remains nearly universal (the "meat paradox"; Bastian and Loughnan (2017)). How does meat-eating behavior survive the resulting cognitive dissonance between people's ethical views and their actual behavior (Rothgerber, 2020)? There are several explanations. First, most individuals in developed countries do not acquire meat by personally rearing animals in intensive factory farm conditions, slaughtering, and preparing them, but rather obtain already processed meat that bears little visual resemblence to the animals from which it came. It is therefore rather easy to implicitly view meat as distinct from animals (Benningstad and Kunst, 2020). This situation is captured well in an episode of The Simpsons that has been used as an intervention to reduce meat consumption (Byrd-Bredbenner et al., 2010), in which Homer Simpson chastises his newly vegetarian daughter: "Lisa, get a hold of yourself. This is lamb, not a lamb!" Some interventions operate simply by reminding the subject of the connection between meat and animals by, for example, displaying photographs of meat dishes alongside photographs of the animals from which they came; these meat-animal reminders seem to consistently reduce meat consumption Kunst and Hohle, 2016, Kunst and Haugestad, 2018; Earle et al., 2019, Tian et al., 2016, da Silva, 2016, Lackner, 2019).

Second, the public is poorly informed about animal welfare conditions on factory farms, and individuals often deliberately avoid information about farm animal welfare, even admitting to doing so when asked explicitly (Onwezen and van der Weele, 2016). Presumably the public avoids information because they anticipate that the results may be upsetting Knight and Barnett, 2008). Thus, interventions that circumvent individuals' cultivated ignorance by graphically describing or depicting conditions on factory farms may provide a "moral shock" that could, for some individuals, lead to dietary change, potentially by triggering 
cognitive dissonance (Rothgerber, 2020, Wrenn, 2013, Jasper and Poulsen, 1995). In principle, animal welfare interventions might be more effective at prompting such dissonance than interventions appealing instead to individual health or the environment, though this point remains speculative (Rothgerber, 2020). However, the use of graphic depictions is controversial, as they might be ineffective or even detrimental in some contexts (Wrenn, 2013).

Third, even when individuals do consider the animal origins of meat and are informed about conditions on factory farms, they may ascribe little or no sentience to animals reared to produce meat or edible animal products (henceforth "farm animals"), limiting the moral relevance of eating meat (Rothgerber, 2020). Indeed, the Cartesian view that animals are automata that do not experience pain, suffering, or emotions, and hence are outside the sphere of moral concern, was once influential in ethical philosophy, though a modern scientific understanding of animal cognition has essentially eliminated this view from scholarly philosophy (Singer, 1995). In fact, individuals may reduce their attributions of mind and sentience post hoc when faced with the dissonance that could otherwise arise from eating meat: subjects randomly assigned to eat beef subsequently reported that cows are less capable of suffering, and they showed less moral concern, than subjects randomly assigned to eat nuts (Loughnan et al. 2010). Interventions that encourage mind attribution to farm animals, for example by asking subjects to imagine the cognitive and affective experiences of a cow, may disarm this dissonance-reduction strategy, thus reducing willingness to eat meat (Amiot et al., 2018). Similarly, other interventions leverage the fact that most people already recognize the sentience of companion animals, such as dogs and cats, and therefore incorporate these animals into their spheres of moral concern, even while excluding farm animals with comparable cognitive abilities and capacity for suffering (Rothgerber, 2020). Interventions targeting this form of dissonance highlight the moral equivalence of farm animals and companion animals, for example by stating: "If the anti-cruelty laws that protect pets were applied to farmed animals, many of the nation's most routine farming practices would be illegal in all 50 states." (Norris and Hannan, 2019) 
Additionally, physical disgust and moral disgust are closely intertwined. Experiencing physical disgust can amplify negative moral judgments, even when the two sources of disgust are conceptually unrelated (e.g., viewing physically disgusting video clips versus judging cheating behavior); conversely, experiencing moral disgust can induce physical disgust (Chapman and Anderson, 2013). Given the powerful impact of physical disgust on food choices (Rozin and Fallon, 1980), evoking moral disgust regarding animal welfare may be a particularly potent means of shaping food choices (Feinberg et al., 2019). Indeed, many animal welfare interventions contain graphic verbal or visual depictions of conditions in factory farms that may themselves be physically disgusting. For example, one intervention (Cordts et al., 2014) describes "crowded conditions [and] pens covered in excrement and germs"; a leaflet that has been studied repeatedly describes sows with "deep, infected sores and scrapes from constantly rubbing against the [gestation crate] bars" and "decomposing corpses [found] in cages with live birds" (Vegan Outreach, 2018). We speculate that such interventions might increase moral disgust by triggering physical disgust. Although we are not aware of studies that directly assess this hypothesis using physically disgusting interventions, it is interesting that even interventions that are not obviously physically disgusting, such as the meat-animal reminders described above, seem to operate in part by increasing physical disgust (Kunst and Hohle, 2016, Earle et al., 2019).

Finally, the connection of animal welfare interventions to an existing social movement encouraging greater consideration of the welfare of farm animals (Singer, 1995) may further trigger "process motivations" for participation (Robinson, 2010, 2017), such that the process of participating in the social movement (e.g., reducing meat consumption due to ethical concerns) may itself be motivating. Participating in social movements can be intrinsically motivating because they provide opportunities for identity development, social interaction and support, perceived belonging, and activities that boost participants' perceptions of collective efficacy (Bandura, 2002). That animal welfare interventions are related to a broader social movement may additionally trigger group- or societal-level changes (e.g., increasing 
public attention, shifting norms regarding meat consumption, or decreasing availability or increased prices of meat) that may alter the social and physical environments to make it easier to sustain the new behaviors (Robinson, 2010, 2017). Whereas behavior-change appeals emphasizing individual health sometimes suffer from high recidivism (Robinson, 2010; Grattan and Connolly-Schoonen, 2012), interventions that instead link behavior to ethical values, self-identity, and existing social movements may be especially potent and long-lasting (Robinson, 2010, 2017; Walton, 2014). Such interventions have, for example, successfully reduced childhood obesity-related behaviors and risk factors for cardiovascular disease and diabetes by appealing to cultural and ethical values in order to increase physical activity, rather than by appealing directly to obesity reduction or other health motivations (Robinson et al., 2003, 2010; Weintraub et al., 2008). Animal welfare interventions might operate similarly.

In theory, then, animal welfare interventions have the potential to be particularly effective by harnessing: (1) multiple general mechanisms of effective behavior interventions (by leveraging social norms and the identifiable victim effect, and by giving implementation suggestions); (2) the unique psychology of meat-eating (by invoking meat-animal reminders, moral shocks triggered by graphic depictions of factory farms, mind attribution, the moral equivalence of farm animals and companion animals, and the physical-moral disgust connection); and (3) the psychological and practical advantages of connection to a social movement. But do these interventions work in practice? We now turn to the present empirical assessment of this question.

\subsection{Objectives of this meta-analysis}

We conducted a systematic review and meta-analysis to address the primary research question: "How effective are animal welfare appeals at reducing consumption of meat?", in which we define "meat" as any edible animal flesh (Section 3.3). We additionally investigated whether interventions' effectiveness differed systematically based on their content, such as types 
of dietary recommendations made, use of verbal, visual, and/or graphic content. or on study characteristics such as length of follow-up and percentage of male subjects (Rozin et al., 2012) (Section 3.5). We evaluated risks of bias in each study (Sections 3.2.4) and conducted numerous sensitivity analyses (Section 3.4). Finally, considering the evidence holistically in light of its methodological strengths and limitations, we discuss what the evidence suggests about the effectiveness of animal welfare interventions (Section 4.1) and give specific recommendations for future research to advance the field (Section 4.3).

\section{METHODS}

See the Reproducibility section for information on the publicly available dataset, code, and materials. All methods and statistical analysis plans were preregistered in detail (https: //osf.io/d3y56/registrations) and subsequently published as a protocol paper (Mathur et al. 2020). In the Supplement, we describe and justify some deviations from this protocol.

\subsection{Systematic search}

Our inclusion criteria were as follows. Studies could recruit from any human population, including from online crowdsourcing websites. Studies needed to assess at least one intervention that was intended to reduce meat consumption or purchase, and interventions needed to include any explicit or implicit mention or portrayal of farm animals, their suffering, their slaughter, or their welfare. Composite interventions, defined as those including both an animal welfare appeal and some other form of appeal (e.g., environmental), were included, though we conducted sensitivity analyses excluding such interventions. Studies needed to include a control group, condition, or time period not subjected to any form of intervention intended to change meat consumption. Last, studies needed to report an outcome regarding the consumption or purchase of meat or all edible animal products, as assessed by a direct

\footnotetext{
${ }^{b}$ We use "graphic content" to refer to explicit, detailed verbal or visual descriptions of conditions on factory farms.
} 
behavioral measure (e.g., the amount of meat that a subject self-served at a buffet), selfreported behavior (e.g., reported meat consumption over the week following exposure to the intervention), or a self-report of intended future behavior (e.g., intended meat consumption over the upcoming week). Although our focus was primarily on meat consumption, we included studies with outcomes related to consumption of all animal products because we anticipated that many interventions designed to reduce meat consumption would in fact make broader recommendations to reduce all animal product consumption and therefore would be assessed using correspondingly broad outcome measures. Further details on inclusion criteria were published previously (Mathur et al., 2020).

In addition to the nascent academic literature, evidence-based nonprofits have conducted numerous studies that have been reported in a separate body of grey literature. We therefore developed sensitive search strategies targeting both the academic literature and the grey literature to identify eligible articles, which could be published or released at any time and written in any language. For non-English articles, we used automated translation to determine eligibility. To search the academic literature, including unpublished dissertations and theses, we collaborated with an academic reference librarian (PAB) to design detailed search strings for each of eight databases (Medline, Embase, Web of Science, PsycInfo, CAB Abstracts, Sociological Abstracts, ProQuest Dissertations \& Theses, and PolicyFile). To search the grey literature and help capture potentially missed academic articles, an author (JP) who is the director of an evidence-based animal welfare research organization designed a three-stage search strategy: (1) we screened The Humane League Labs' existing internal database compiling relevant literature, including both academic studies and grey literature; (2) we screened the websites of 24 relevant nonprofits; and (3) we posted a bibliography of literature identified in the first two stages on relevant forums in the animal advocacy research community to solicit additional leads to studies.9 All methods are detailed in the Supplement.

\footnotetext{
${ }^{\mathrm{c}}$ Because a previous systematic review (Bianchi et al. 2018a) suggested a paucity of academic study on animal welfare appeals to reduce meat consumption, we did not try to identify academic counterparts to these forums. However, participants in the animal advocacy research forums and nonprofits we contacted include numerous academics and researchers with extensive academic training who follow the relevant academic
} 
Final searches were conducted on January 17, 2020 for the academic literature and November 20, 2019 for the grey literature.

We reviewed articles and managed data using the software applications Covidence (Covidence Development Team, 2019) and Microsoft Excel. Each article retrieved from an academic database first underwent title/abstract screening conducted independently by at least two reviewers from among DBR, JN, and MBM. In this stage, reviewers excluded only articles that clearly failed the inclusion criteria. Articles receiving an "include" vote from either or both reviewers proceeded to a full-text screening, during which at least two reviewers independently assessed inclusion criteria in detail. We resolved 9 conflicts between reviewers through discussion or adjudication by other authors. Interrater reliability for inclusion decisions was greater thand Cohen's $\kappa=0.71$. Grey literature articles that JP identified as potentially eligible underwent the same full-text dual review as academic articles. We refer to articles ultimately judged to meet all inclusion criteria as "eligible".

\subsection{Data extraction}

Basic descriptive characteristics of studies. For each study, we extracted 33 basic descriptive characteristics regarding, for example, the subject population, location, intervention, and outcome. All extracted characteristics are enumerated in the Supplement. One of the extracted variables indicated whether the study was borderline with respect to the inclusion criteria, a classification made through discussion amongst the review team. For example, one "meat-animal reminder" intervention consisting of a photograph of a pork roast with

literature closely, and indeed, we obtained a number of candidate academic journal articles in this manner.

${ }^{\mathrm{d}}$ Because the three raters rated only partly overlapping subsets of studies, we calculated $\kappa$ for each of the three pairs of raters and report the mean $\kappa$ across the three pairs of raters, weighted by the number of articles they both rated. We could only estimate a lower bound on each pairwise $\kappa$ because a large number of apparent "conflicts" in fact reflected reviewers' different handling of duplicate articles. That is, we initially instructed reviewers to vote "exclude" on duplicate articles, but we then became aware that a large number of articles reporting on duplicated samples provided additional information that would be useful in extracting study characteristics. (This was especially common among the unpublished studies.) We then instructed reviewers to instead vote "include" on all such articles, which created some spurious "conflicts" that were not clearly differentiated by our data extraction software. The 9 conflicts we report above represent only the real conflicts not arising from duplicate articles. 
the head still attached (versus a headless roast in the control condition) was classified as borderline because it was difficult to judge whether the intervention was about animal welfare. As described in Section 2.3, we conducted sensitivity analyses excluding borderline studies.

Hypothesized effect modifiers. Also among the extracted variables were 13 hypothesized effect modifiers. [ We categorize the effect modifiers into 8 "coarse" intervention characteristics, which we could code for nearly all studies, versus 5 "fine-grained" intervention characteristics, which we could code for only the $k=80$ studies for which we had access to all intervention materials or to detailed descriptions of their content. The coarse intervention characteristics were: (1) whether the intervention contained text; (2) whether the intervention contained visuals; (3) whether the intervention contained graphic visual or verbal depictions of factory farm conditions; (4) the nature of the recommendation made in the intervention ("go vegan", "go vegetarian", "reduce consumption", a mixed recommendation [e.g., recommendations to either reduce or eliminate meat consumption], or no recommendation); (5) lasted longer than 5 minutes; (6) whether the outcome was measured 7 or more days after the intervention (to capture possible decays in intervention effects over time); (7) the percentage of male subjects in the study (because meat-eating may be closely intertwined with masculine identity in Western cultures (Ruby, 2012, Rozin et al., 2012, Rothgerber, 2020); and (8) subjects' mean or median agef

The fine-grained intervention characteristics described whether the intervention: (1) used mind attribution by describing a farm animal's inner states; (2) described social norms in favor of reduced meat consumption; (3) used the identifiable victim effect by giving a proper-noun name to a farm animal; (4) described or depicted pets (i.e., companion animals that typically live in people's houses) with or without explicitly comparing them to farm

${ }^{\text {e}}$ Our preregistration and protocol paper (Mathur et al. 2020) gave non-exhaustive examples of candidate effect modifiers we might investigate, but did not formally preregister this list.

${ }^{\mathrm{f}}$ It would have been informative to also investigate possible effect modification by geographic location. However, given the high rate of missing data on this variable and its sparse distribution across countries (Table 1), the meta-regression model was not statistically estimable with the inclusion of this covariate, even when it was coarsely coded as "North America" versus "elsewhere". 
animals; and (5) gave implementation suggestions in the form of describing or depicting a specific plant-based meal, restaurant dish, or recipe.

Risk-of-bias characteristics. We also extracted 9 characteristics related to the study's risks of bias, including design characteristics (e.g., randomization), missing data, analytic reproducibility and preregistration practices, exchangeability of the intervention and control conditions (i.e., avoidance of statistical confounding through randomization and minimization of differential dropout), avoidance of social desirability bias (e.g., by keeping subjects naïve to the purpose of the intervention), and external generalizability (i.e., the extent to which results are likely to apply to subjects without pre-existing motivations to reduce meat consumption or purchase). Details on the development, contents, and use of our risk-of-bias tool are provided in the Supplement.

Quantitative data extraction. We extracted quantitative data for the meta-analysis as follows. For each eligible article that reported sufficient statistical information, the statistician (MBM) used either custom-written $\mathrm{R}$ code or the $\mathrm{R}$ package metafor (Viechtbauer, 2010) to extract the point estimate(s) and variance estimate(s) most closely approximating the treatment effect of the animal welfare appeal on the risk ratio $(R R)$ scale (see Supplement for details). We synchronized the directions of all studies' estimates such that risk ratios greater than 1 represented reductions in meat consumption or purchase. When relevant statistics were not reported, we hand-calculated them from available statistics, plots, or publicly available datasets as feasible, or made repeated attempts to contact study authors. Articles reporting multiple point estimates on separate subject samples (even those sharing a control group) could contribute all of these point estimates to the analyses; further details on how we handled articles reporting on multiple eligible interventions were published previously (Mathur et al., 2020). For studies that reported multiple outcomes (e.g., consumption or purchase of all meat, of all edible animal products, and of specific subsets of meats), we used the outcome most closely matching the intended scope of the intervention. For example, 
we used the outcome "pork consumption" for an intervention specifically targeting pork consumption (Anderson, 2017) and used the outcome "all animal product consumption" for an intervention that recommended going vegan (Bertolaso, 2015).

Although meta-analysts sometimes extract all eligible outcomes from each paper and average them within studies (Sutton et al., 2000), we correctly anticipated that most studies with multiple eligible food outcomes would be those whose primary outcome was a composite measure of total meat or total animal product consumption and whose other eligible food outcomes were subscales of this composite, representing consumption of specific meats and animal products. Averaging these estimates within a given study would therefore yield a point estimate that would be essentially equivalent to the composite itself, but with the additional limitation that its variance would not be estimable without information on the full correlation structure of the various subscales. When possible, to reduce the possibility of our choosing which estimates to include in a biased manner, we made all decisions about how we would calculate each study's point estimate, and for which outcome, according to these and other detailed rules (see Supplement) before we actually calculated the estimate. However, doing so was not always feasible (e.g., for studies that directly reported the point estimates we needed to calculate).

\subsection{Statistical analysis methods}

Main analyses. The statistician (MBM) conducted all statistical analyses in R (R Core

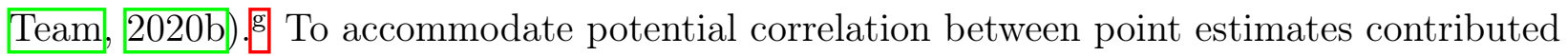
by the same article and to obviate distributional assumptions, we fit a robust semiparametric meta-analysis model using the $\mathrm{R}$ package robumeta (Hedges et al., 2010, Fisher and Tipton,

${ }^{\mathrm{g}}$ We used the following R packages: Canty and Ripley (2020); Ooi et al. (2020); Kuhn et al. (2020); Wickham et al. (2020); Wickham (2016); Rodriguez-Sanchez (2018); Wolak et al. (2012); Gamer et al. (2019); Viechtbauer (2010); Mathur et al. (2019); Wickham (2011a); Mathur and VanderWeele (2020a); Neuwirth (2014); Wickham and Bryan (2019); Fisher and Tipton (2015); Yoshida (2020); Wickham (2011b); Wickham et al. (2019); Coburn and Vevea (2019); Dahl et al. (2019); Fox and Weisberg (2019); Dowle and Srinivasan (2019); R Core Team (2020a); Bates et al. (2015); Bulow (2016); Zeileis (2004); Lüdecke (2020); Wickham (2019. 2011b); Wickham and Henry (2020); Wickham et al. (2019); Tierney et al. (2020). 
2015). For the primary analysis, we included all eligible and borderline-eligible studies and estimated the average effect size with a $95 \%$ confidence interval and $p$-value, the standard deviation of the true population effects $(\widehat{\tau})$, and metrics that characterize evidence strength when effects are heterogeneous. Regarding the latter, we estimated the percentage of true population effects that were stronger than various effect-size thresholds representing all effects in the beneficial direction (i.e., $R R>1$ ) and more stringently representing only effects that might be considered to be meaningfully large by two different criteria (i.e., $R R>1.1$ or $R R>1.2)$ (Mathur and VanderWeele, 2019, 2020b). 1]

We also estimated the percentage of true population effects with risk ratios less than 1 and alternatively less than 0.90 , representing unintended detrimental effects of the interventions (Mathur and VanderWeele, 2019, 2020b). Taken together, these percentage metrics can help identify if: (1) there are few meaningfully large effects despite a "statistically significant" meta-analytic mean; (2) there are some large effects despite an apparently null meta-analytic mean; or (3) strong effects in the direction opposite of the meta-analytic mean also regularly occur (Mathur and VanderWeele, 2019, 2020b). As a hypothesis-generating method to help identify the individual interventions that appear most effective, we estimated the true population effect size in each study using nonparametric calibrated estimates (Wang and Lee, 2019). Intuitively, the calibrated estimates account for differences in precision between studies by shrinking each point estimate toward the meta-analytic mean, such that the least precise studies receive the strongest shrinkage toward the meta-analytic mean.

\footnotetext{
${ }^{\mathrm{h}}$ We use the term "true population effects" to refer to population parameters, rather than to point estimates with statistical error. "True" does not indicate that these effect sizes are free from bias due, for example, to attrition or social desirability bias.

${ }^{i}$ As previously recommended (Hill et al. 2008), we chose these thresholds with reference to the effect sizes of other health behavior interventions; for example, general nutritional "nudges" (not specific to meat consumption or animal welfare) produce average effect sizes (Arno and Thomas, 2016) of approximately $R R=1.15$, and graphic warnings on cigarette boxes increase short-term intentions to quit by approximately $R R=1.14$ upon conversion from the odds ratio scale (Brewer et al., 2016; VanderWeele, 2017, VanderWeele, 2019). These effect sizes are close to our two chosen thresholds. Nevertheless, these thresholds are somewhat arbitrary; therefore, Figure S3 shows the percentage of effects as a continuous function of the threshold chosen.
} 
Publication bias. We assessed publication bias using selection model methods (Vevea and Hedges, 1995), sensitivity analysis methods (Mathur and VanderWeele, 2020c), and the significance funnel plot (Mathur and VanderWeele, 2020c). These methods assume that the publication process favors "statistically significant" (i.e., $p<0.05$ ) and positive results over "nonsignificant" or negative results, a typically reasonable assumption that also conforms well to empirical evidence on how publication bias operates in practice (Jin et al., 2015; Mathur and VanderWeele, 2020c). We used the sensitivity analysis methods to estimate the meta-analytic mean under hypothetical worst-case publication bias (i.e., if "statistically significant" positive results were infinitely more likely to be published than "nonsignificant" or negative results). These methods, unlike the selection model, also accommodated the point estimates' non-independence within articles and did not make any distributional assumptions (Mathur and VanderWeele, 2020c).

Other sensitivity analyses. As further sensitivity analyses, we conducted meta-analyses within 9 separate subsets of studies, namely: (1) excluding studies with borderline eligibility (leaving $k=91$ analyzed studies); (2) excluding studies with composite interventions ( $k=52$ analyzed); (3) excluding studies that measured intended behavior rather than directly measured or self-reported behavior ( $k=43$ analyzed); (4) including only studies judged to be at the lowest risk of bias as described above $(k=12)$; (5) including only randomized studies $(k=75) ;(6)$ including only studies that were preregistered and had open data $(k=21) ;(7)$ including only published studies $(k=17)$; (8) including only unpublished studies $(k=83)$; and (9) excluding one study (FIAPO, 2017) with a very large point estimate and also a very large standard error, visible in Figure 1 $(k=99$ analyzed). The first 2 subset analyses were preregistered; the subsequent ones were introduced post hoc. We also conducted a simple post hoc analysis that assessed the sensitivity of our results to potential social desirability bias, conceptualized as bias in which subjects in the intervention group under-report meat and animal product consumption more than subjects in the control group VanderWeele and 
Li, 2019). We ultimately did not conduct a preregistered sensitivity analysis in which we would have excluded interventions targeting consumption of only a specific type of meat; this proved infeasible because of the small number of interventions that clearly targeted a specific meat or meats.

Meta-regression on hypothesized effect modifiers. As a secondary analysis, we used robust meta-regression (Hedges et al., 2010) to investigate intervention and study characteristics associated with increased or decreased effectiveness. The meta-regressive covariates were the hypothesized effect modifiers listed in Section 2.2. As described there, we could code the 5 fine-grained intervention characteristics (namely, use of mind attribution, social norms, the identifiable victim effect, depictions of pets, and implementation suggestions) for 80 studies. Additionally, because many of these components were used only in interventions that contained text, these 5 covariates were highly collinear with the covariate indicating that the intervention contained text. We therefore fit two meta-regression models: (1) a "coarse" model that included only the coarse intervention characteristics listed in Section $2.2(k=$ 86 studies with complete data on the effect modifiers); and (2) a "fine-grained" model containing all fine-grained and the coarse intervention characteristics except the presence of text ( $k=80$ studies). For each model, we included the covariates in the meta-regression simultaneously rather than in separate univariable models because they were fairly highly correlated (Supplementary Table S5).

Metrics of societal impact. We had preregistered an additional type of secondary analysis in which we intended to express intervention effectiveness using metrics that more directly characterize societal impact, such as the estimated reduction in human all-cause mortality events, in the number of animals reared for consumption, and in greenhouse gas emissions. However, limitations in outcome measurement and follow-up duration in the meta-analyzed studies ultimately precluded these analyses; we return to this issue in Section 4.3 . 


\section{RESULTS}

\subsection{Results of search process}

Supplementary Figure S1 is a PRISMA flowchart depicting the search results. After removing duplicate articles, we screened 4,139 articles from the academic database searches and an additional 81 articles identified from manual searches of the grey literature and the academic literature. We assessed 144 full-text articles for eligibility. In full-text screening, we removed 96 articles that failed inclusion criteria, that reported insufficient information to determine eligibility and for which we could not obtain full texts, or that analyzed the same subject sample as an existing article (e.g., because they were re-analyses of an existing dataset). We thus found that 48 articles met the inclusion criteria. Of these, 7 could not be meta-analyzed because we could not obtain the relevant point estimates from publicly available data, from the paper, or from repeated attempts to contact the authors. (Characteristics of these eligible, but not analyzed, studies are described qualitatively in Supplementary Table S3.)

Of the 41 articles with available statistics, 7 met our preregistered inclusion criteria but were excluded from the main analysis because they reported on a specific unanticipated study design at very high risk of bias. These studies assessed the effectiveness of "challenges" or "pledges" in which, for example, subjects try to follow a vegan diet for one month. These studies shared two key design features: (1) subjects were not specifically recruited, but rather were individuals who had chosen to sign up for the challenge and who therefore may have been already highly motivated to reduce their consumption; and (2) there was no separate control group, but rather the challenge's effectiveness was assessed by comparing within-subject changes in consumption after versus before the challenge. The combination of these two features is highly problematic: subjects with a strong pre-existing motivation to reduce consumption may have done so regardless of whether they took the challenge, and the lack of a separate control group precludes estimation of a valid treatment effect of the challenge even among this subset of highly motivated subjects. These studies also typically provided 
very limited statistical summaries; in many cases, we would not have had enough information to calculate an effect size that was statistically comparable to the risk ratios we estimated for other studies. For these reasons, we made a post hoc decision during data extraction to exclude studies with both of these features (subject self-selection and lack of a separate control group) from the main analysis. However, in keeping with our preregistered inclusion criteria, we report results that include these "self-selected within-subjects" (SSWS) studies in Section 3.6. Therefore, after excluding the 7 articles reporting on SSWS studies, main analyses included 34 articles, totaling 100 point estimates.

\subsection{Characteristics of included articles and studies}

Table 1 summarizes studies' basic characteristics. We meta-analyzed data from 34 articles; $24 \%$ were published in peer-reviewed journals, and all others were dissertations, theses, conference proceedings, or reports by nonprofits. The chronologically first study (ByrdBredbenner et al. 2010) was published in 2010. The articles contributed a total of 100 point estimates, which were estimated using a total of 24,817 subjects. We will refer to these 100 estimates as "studies" to distinguish them from "articles". We obtained $53 \%$ of statistical estimates from publicly available datasets; $28 \%$ by manually calculating statistics from information reported in the articles' text, tables, or figures; and the remaining $19 \%$ from datasets provided by the study's authors.

Many studies (53\%) reported multiple food outcomes that were potentially eligible for inclusion; as described in Section 2.2. we extracted statistics for only the outcome most closely matching the scope of the intervention. For all but one article, the outcome we extracted (e.g., consumption of all meats) was a composite of some or all of the additional food outcomes (e.g., consumption of specific categories of meats), as we had anticipated. For the remaining article (Caldwell, 2016), the primary and additional outcomes were pork consumption and egg consumption respectively, and the latter was measured because the study also contained a non-eligible intervention regarding egg production. Given the large 
number of studies, we detail their individual characteristics, along with risks of bias ratings, point estimates, variance estimates, and additional food outcomes, in a publicly available spreadsheet (https://osf.io/8zsw7/; see Supplement for codebook).

\subsubsection{Subjects}

The median analyzed sample size per article was 522 subjects $\left(25^{\text {th }}\right.$ percentile: $235 ; 75^{\text {th }}$ percentile: 802). (We report the sample sizes by article rather than by study to avoid double-counting control subjects whose data contributed to multiple point estimates from the same article.) Studies were conducted in at least 11 countries (Canada, China, the Czech Republic, Ecuador, France, Germany, India, the Netherlands, Portugal, Scotland, and the United States) that represented a diverse range of geographical regions as well as cultural characteristics that may be relevant to meat consumption, including affluence, traditional cuisines, and religious and ethical traditions. The median percentage of male subjects was $43 \%$, and the median of studies' average ages was 33.7 years. $13 \%$ of studies recruited only undergraduates, including $2 \%$ (2 studies) that specifically recruited social sciences undergraduates. $77 \%$ did not specifically recruit undergraduates, and the remaining $10 \%$ recruited both undergraduate and non-undergraduate samples.

\subsubsection{Interventions}

Examples of typical interventions include, not exhaustively, providing informational leaflets about factory farming conditions (Norris and Hannan, 2019), showing photographs of meat dishes accompanied by photographs of the animals they came from (Kunst and Hohle, 2016), and providing mock newspaper articles (Macdonald et al., 2016). Details on the many other interventions represented in our meta-analysis are provided in the aforementioned publicly available spreadsheet of study characteristics. Below, we report on studies' characteristics using percentages that count missing data as its own category, so percentages may add up to less than $100 \%$. Most studies' interventions contained text (83\%), contained visuals (e.g., 
photographs, infographics, or videos) (62\%), contained graphic verbal or visual depictions of welfare conditions in factory farms (61\%), and used mind attribution (65\%). Relatively fewer interventions invoked social norms (29\%), identified a named victim (21\%), depicted pets (32\%), and gave implementation suggestions (29\%), though these relatively lower percentages also reflect missing data on these variables when we did not have access to all intervention materials.

Most interventions (52\%) made appeals only regarding animal welfare, with the remaining interventions also appealing to, for example, individual health or environmental concerns. The interventions were typically quite brief in duration: we estimated that $66 \%$ lasted less than 5 minutes, though there was a wide range, with some interventions lasting only about 30 seconds and others lasting over 90 minutes. Interventions also varied considerably in their use of explicit recommendations to the viewer: $13 \%$ recommended reducing meat consumption, $9 \%$ recommended going vegetarian, $7 \%$ recommended going vegan, and $24 \%$ made some combination of these recommendations. Another $43 \%$ of interventions made no explicit recommendation, instead simply describing or depicting animal welfare in a manner that was intended to reduce meat consumption. The overwhelming majority (98\%) of interventions were not personally tailored (i.e., their content was the same for all subjects regardless of personal characteristics); the few tailored interventions were from a single article (FIAPO, 2017) in which the interventions' contents differed for currently vegetarian subjects versus for currently non-vegetarian subjects.

\subsubsection{Outcomes}

Throughout this section, we describe the outcomes used in our own analysis based on the considerations described in Section 2, which sometimes differed from the main outcome reported in the article, especially when raw data were available. Nearly all studies (96 studies; $96 \%$ ) assessed consumption of meat or animal products, with the remaining studies instead assessing purchasing. $57 \%$ of studies assessed subjects' intended future behavior, another 
$41 \%$ of studies assessed subjects' self-reported behavior, and the remaining $2 \%$ (2 studies) used direct objective measures of subjects' behavior (i.e., the amount eaten of an offered sample of ham (Anderson and Barrett, 2016) or the percentage of actually purchased meals that contained meat (Schwitzgebel et al., 2019)). About half of studies (53\%) measured outcomes immediately after exposure to the intervention. Among the studies that measured outcomes after some delay, the median and maximum follow-up lengths were 39 and 120 days, respectively. For $71 \%$ of studies, our extracted point estimates were risk ratios of low versus high meat or animal product consumption i] defined in absolute terms (e.g., being below versus above the baseline median consumption); the remaining estimates were risk ratios of reducing consumption relative to one's own previous consumption.

\subsubsection{Design quality, reproducibility, and risks of bias}

Table 2 summarizes study characteristics related to the methodological strength of study design, analytic reproducibility, potential for selective reporting, and risks of bias. The table presents these characteristics for all studies, for published studies, and for unpublished studies. Overall, $75 \%$ of point estimates were from randomized studies $\}^{\mathbb{E}}$ (with randomization occurring between subjects, within subjects, or by clusters of subjects); the remaining estimates were from nonrandomized designs with a separate control group or in which subjects' meat consumption was assessed before and after the intervention. Regarding analytic reproducibility and the potential for selective reporting, $53 \%$ of studies had publicly available data, $22 \%$ had publicly available analysis code, and $25 \%$ had been preregistered. The median percent of missing data at the longest follow-up time point was $7.5 \%$, but $23 \%$ of studies did not report on missing data. As described above, nearly all studies (98\%) measured outcomes based on subjects' self-reports of behavior or of future intentions, rather than based on direct behavioral

\footnotetext{
${ }^{\mathrm{j}}$ As described in Section 2.2 we defined the outcome in terms of animal product consumption rather than meat consumption when the intervention itself targeted animal product consumption.

${ }^{\mathrm{k}}$ When a study did not explicitly mention randomization, we assumed it was not randomized.

${ }^{l}$ For studies in which outcomes were measured immediately after the intervention and that did not report on missing data, we assumed there was none.
} 
measures. We gave "low" or "medium" risk-of-bias ratings (versus "high" or "unclear") to $61 \%$ of studies regarding exchangeability of the intervention and control groups, to $27 \%$ regarding social desirability bias, and to $64 \%$ of studies regarding external generalizability $\mathrm{m}$ Published studies appeared to be at lower risk of bias than unpublished studies on most criteria, except that unpublished studies had notably better analytic reproducibility and preregistration practices (Table 2). Post hoc, we defined studies with the lowest risk of bias overall as those that were randomized, had less than $15 \%$ missing data, and had "low" or "medium" risks of bias for exchangeability and social desirability bias 12 studies, contributed by 6 unique articles (Anderson and Barrett, 2016; Earle et al., 2019; Kunst and Hohle, 2016; Kunst and Haugestad, 2018, Lackner, 2019, Tian et al., 2016), were identified as being at lowest risk of bias.

\subsection{Main analyses}

The overall pooled risk ratio was 1.22 (95\% CI: [1.13, 1.33]; $p<0.0001)$, indicating that on average, interventions increased by $22 \%$ a subject's probability of intending, self-reporting, or behaviorally demonstrating low versus high meat consumption, compared to the control condition. Most articles (71\%) contributed more than one point estimate to the metaanalysis, and point estimates were moderately correlated within articles (intraclass correlation coefficient $=0.44)$.

Figure 2 shows point estimates, 95\% confidence intervals [CI], and calibrated estimates for each study. Interventions' effect sizes appeared fairly heterogeneous across studies, with

\footnotetext{
${ }^{\mathrm{m}}$ However, these results regarding exchangeability, social desirability bias, and external generalizability should be interpreted cautiously because interrater reliability for these characteristics was fairly low, reflecting both their somewhat subjective nature and the fact that many studies lacked relevant methodological details (Supplement).

${ }^{\mathrm{n}}$ We created this designation in order to compare the relatively strongest studies in our meta-analysis to the others as a sensitivity analysis, rather than to claim that the former studies were, in absolute terms, at low risk of bias on all domains. This designation was inherently somewhat arbitrary and considered only a subset of risk-of-bias variables that were most directly related to studies' ability to unbiasedly estimate a causal treatment effect, even if that treatment effect may apply only to certain types of subjects, may apply only to intended behavior, or may apply only to behavior immediately after the intervention. If we had required high ratings on every risk-of-bias variable simultaneously, too few studies would have received the designation for us to conduct the sensitivity analysis.
} 
an estimated standard deviation of the log-risk ratios of $\widehat{\tau}=0.12$. Supplementary Figure S2 shows the estimated distribution of true population effects across all studies. Despite this heterogeneity, the interventions' effects were overwhelmingly in the beneficial direction (i.e., reducing rather than increasing meat consumption, purchase, or relevant intentions), with an estimated $83 \%$ (95\% CI: [72\%, 91\%]) of true risk ratios above 1. Upon more stringently considering only risk ratios of at least 1.1, or alternatively 1.2, as being meaningfully large, we estimated that $71 \%(95 \%$ CI: $[59 \%, 80 \%])$ and $53 \%(95 \%$ CI: $[38 \%, 64 \%])$ of effects were stronger than these two thresholds, respectively. Supplementary Figure S3 displays the estimated percentage of risk ratios above various other thresholds. Considering effects in the detrimental direction (i.e., interventions that "backfired" by increasing rather than decreasing meat consumption relative to the control condition), we estimated that $17 \%$ (95\% CI: $[6 \%, 27 \%])$ of interventions had true population risk ratios below 1 . Very few interventions produced risk ratios smaller than 0.90 in the detrimental direction (4\%; 95\% CI: [0\%, 12\%]).

The 10 studies with the largest calibrated estimates (i.e., the top 10\%) used interventions consisting of: brief factual passages that graphically described and/or visually depicted conditions on factory farms (Cordts et al., 2014, Reese, 2015) or fish farms (Rouk, 2017), sometimes combined with health or environmental appeals (Reese, 2015); a 16-page leaflet containing detailed information and graphic portrayals of conditions on factory farms along with health appeals and implementation suggestions ("Even If You Like Meat" leaflet; Norris and Roberts (2016)); a factual mock newspaper article with graphic photographs and descriptions of gestation crates along with discussion of legislation to ban their use (Caldwell. 2016); a 3-minute virtual reality video graphically depicting conditions on factory farms (FIAPO, 2017); and meat-animal reminders consisting of photographs of meat dishes portrayed alongside photographs of the animals they came from (Earle et al., 2019). Nine of these 10 studies assessed outcomes related to intended behavior, and the remaining study assessed self-reported behavior (Norris and Roberts, 2016). 


\subsection{Sensitivity analyses}

\subsubsection{Publication bias}

The meta-analytic mean corrected for publication bias (Hedges, 1992) was 1.33 (95\% CI: [1.22, $1.45] ; p<0.0001)$. Thus, this estimate was in fact somewhat larger than the uncorrected metaanalytic mean of 1.22 , though the estimates had substantially overlapping confidence intervals. This result suggests that publication bias was likely mild and did not meaningfully affect results. As a post hoc analysis, we fit the selection model to unpublished and to published studies separately, yielding similar conclusions. The sensitivity analyses for publication bias indicated that even under hypothetical worst-case publication bias (i.e., if "statistically significant" positive results were infinitely more likely to be published than "nonsignificant" or negative results), the meta-analytic mean would decrease to 1.07 (95\% CI: [1.02, 1.13]) but would remain positive and with a confidence interval bounded above the null. This worst-case estimate arises from meta-analyzing only the 75 observed "nonsignificant" or negative studies and excluding the 25 observed "significant" and positive studies (Mathur and VanderWeele, 2020c). As a graphical heuristic, the significance funnel plot in Figure 1 relates studies' point estimates to their standard errors and compares the pooled estimate within all studies (black diamond) to the worst-case estimate (grey diamond). When the diamonds are close to one another or the grey diamond represents a positive, nonnegligible effect size, as seen here, the meta-analysis may be considered fairly robust to publication bias (Mathur and VanderWeele, 2020c). Taken together, the results from the selection model and from the sensitivity analysis suggest that publication bias appeared negligible and not likely to substantially attenuate the results.

\subsubsection{Social desirability bias}

We conducted a simple sensitivity analysis (VanderWeele and Li (2019); Mathur and VanderWeele (2020b); see Supplement for methodological details) that characterizes how severe 
social desirability bias would have to have been in the meta-analyzed studies in order for: (1) the pooled point estimate of $R R=1.22$ to be entirely attributable to social desirability bias (i.e., such that the interventions in fact had no effect on actual consumption on average); or (2) the percentage of true population effects stronger than $R R=1.1$ to be reduced from our estimated $71 \%$ to only $10 \%$. Specifically, the sensitivity analyses characterize the hypothetical severity of social desirability bias as how strongly the interventions affected subjects' reported consumption independently of their effects on subjects' actual consumption (VanderWeele and Li, 2019). For example, this form of social desirability bias could arise in a study in which the intervention did not affect subjects' actual consumption at all, but did induce them to under-report meat consumption more than they otherwise would, thus yielding a spurious intervention effect estimate. If there were no social desirability bias of this form, the interventions would affect reported consumption exclusively via their effects on actual consumption.

Another plausible form of social desirability bias could arise if subjects were to systematically under-report meat consumption, but by a similar degree in the intervention and control groups (e.g., Hebert et al. (1995); Neff et al. (2018); Rothgerber (2020)). Critically, this form of under-reporting would in general leave estimates of intervention effects either unbiased or would bias them toward, rather than away from, the null (Rothman et al. 2008). Hence, this form of under-reporting is of less concern than the type of differential social desirability bias that we considered in the sensitivity analyses, which could bias the estimates away from the null (Rothman et al., 2008).

The sensitivity analyses indicated that, for the observed $R R=1.22$ to be entirely attributable to social desirability bias, the interventions would have needed to increase subjects' probability of reporting low meat consumption, entirely independently of their potential effects on actual consumption, by at least $22 \%$ (95\% CI: [17\%, 29\%]) on average. If the severity of social desirability bias were approximately the same for all studies, then to reduce the percentage of true population effects stronger than $R R=1.1$ from our estimated 
$71 \%$ to only $10 \%$ would require that each intervention had increased subjects' probability of reporting low meat consumption, independent of its effects on actual consumption, by at least $37 \%$ (95\% CI: [31\%, $55 \%])$.

\subsubsection{Subset analyses}

The 9 subset analyses described in Section 2.3 yielded point estimates that were typically close to the main estimate of 1.22 and that were never smaller than 1.09 (ranging from 1.09 for preregistered studies with open data to 1.35 when restricting the analysis to published studies; see Table 3). Some estimates had wide confidence intervals due to the inclusion of only a small number of studies. All subset analyses corroborated the conclusion that a large majority of true population effects (at least 70\%) were in the beneficial direction, and 7 of 9 analyses corroborated the conclusion that a majority of true population effects were greater than $R R=1.1$.

\subsection{Secondary analyses}

As described in Section 2.3, we fit two meta-regression models. In the coarse model $(k=86$ studies), the inclusion of the covariates in the model reduced the heterogeneity estimate from $\widehat{\tau}=0.12$ to 0.07 , suggesting that these covariates together predicted a moderate amount of the heterogeneity seen in the main analysis. Table 4 shows risk ratio estimates for each effect modifier, interpretable as the ratio by which the average intervention effect increased in studies in which the effect modifier was present versus absent. In general, these candidate effect modifiers were not strongly associated with effect sizes, although the confidence intervals sometimes indicated considerable uncertainty. Results were interesting regarding the type of recommendation made: studies of interventions making a "go vegan" recommendation appeared to have larger effects than those of interventions making no recommendation (effect modification $R R=1.31 ; 95 \%$ CI: [1.06, 1.62]; $p=0.03)$, and point estimates in studies whose interventions recommended "going vegetarian" (1.03) or "reducing consumption" (1.00) 


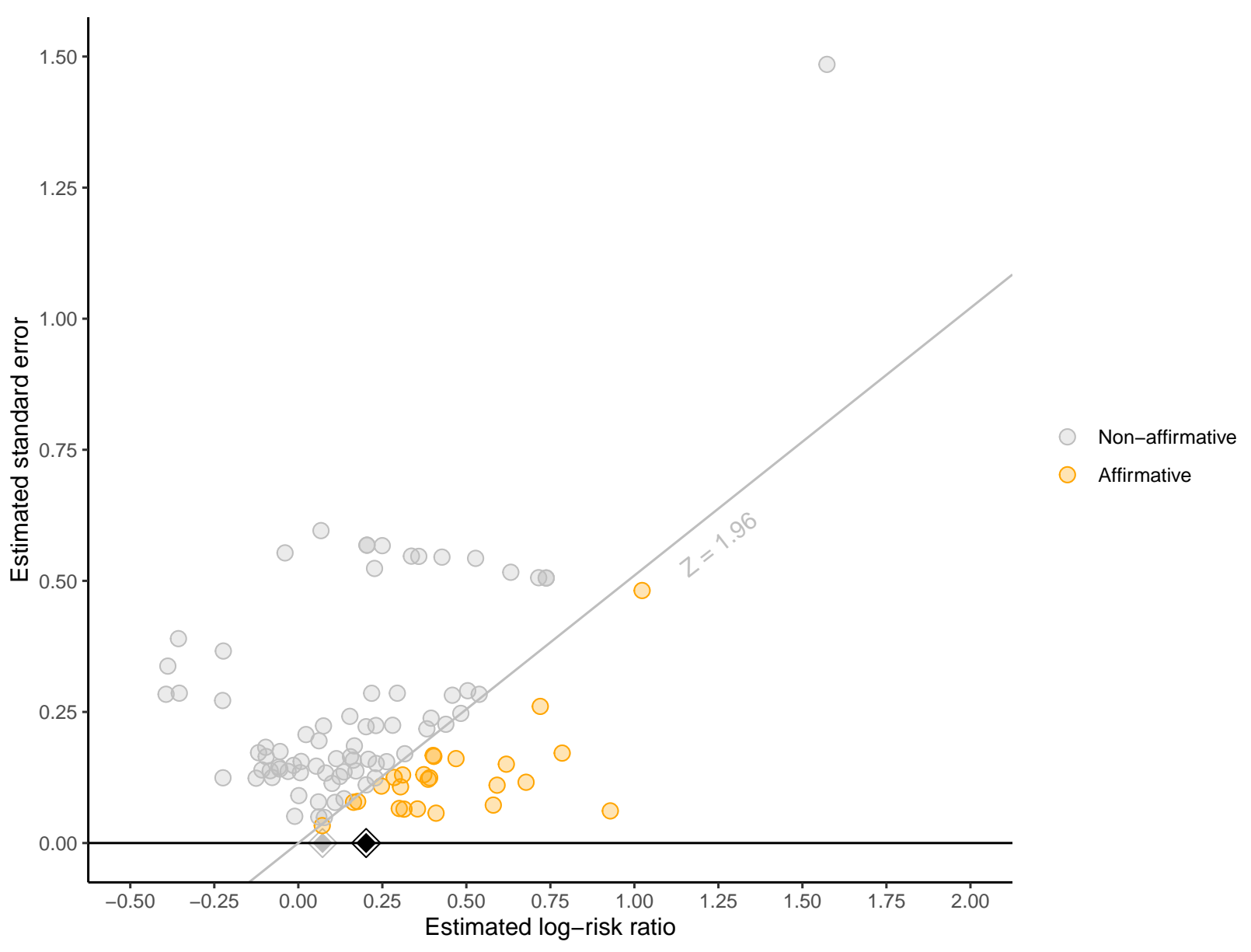

Figure 1: Significance funnel plot displaying studies' point estimates versus their estimated standard errors. Orange points: affirmative studies ( $p<0.05$ and a positive point estimate). Grey points: nonaffirmative studies ( $p \geq 0.05$ or a negative point estimate). Diagonal grey line: the standard threshold of "statistical significance" for positive point estimates; studies lying on the line have exactly $p=0.05$. Black diamond: main-analysis point estimate within all studies; grey diamond: worst-case point estimate within only the nonaffirmative studies. 
heuristically suggested some degree of dose-response such that studies with broader-scoped recommendations (e.g., "go vegan" versus "reduce meat consumption") typically had larger effects. Regarding other study characteristics, studies measuring outcomes after a relatively long delay ( $\geq 7$ days) typically had smaller effect sizes (effect modification $R R=0.81 ; 95 \%$ CI: $[0.68,0.97] ; p=0.03)$, which could reflect decays in intervention effectiveness over time. See the Discussion for important guidance on the interpretation of these meta-regressive results. Supplementary Figures S4-S6 plot individual studies' calibrated estimates versus the interventions' durations, studies' lengths of follow-up, and average ages.

In the fine-grained meta-regression model ( $k=80$ studies; Table 5$)$, the point estimates for the fine-grained intervention components were again usually close to the null, with the possible exception of using implementation suggestions (effect modification $R R=1.24 ; 95 \%$ CI: $[0.85,1.80] ; p=0.22)$. In most cases, the confidence intervals were fairly wide. Estimates

for the other intervention components (Table 5) were usually similar to those in the coarse model.

\subsection{Self-selected within-subjects studies}

As described in Section 3.1. self-selected within-subjects (SSWS) studies as defined in Section 3.1 were excluded post hoc from main analyses, though they did meet our preregistered inclusion criteria. When we instead included the 8 SSWS studies from which we could obtain point estimates, the meta-analytic point estimate was somewhat larger than in main analyses (Table 3), but overall conclusions were not affected. 


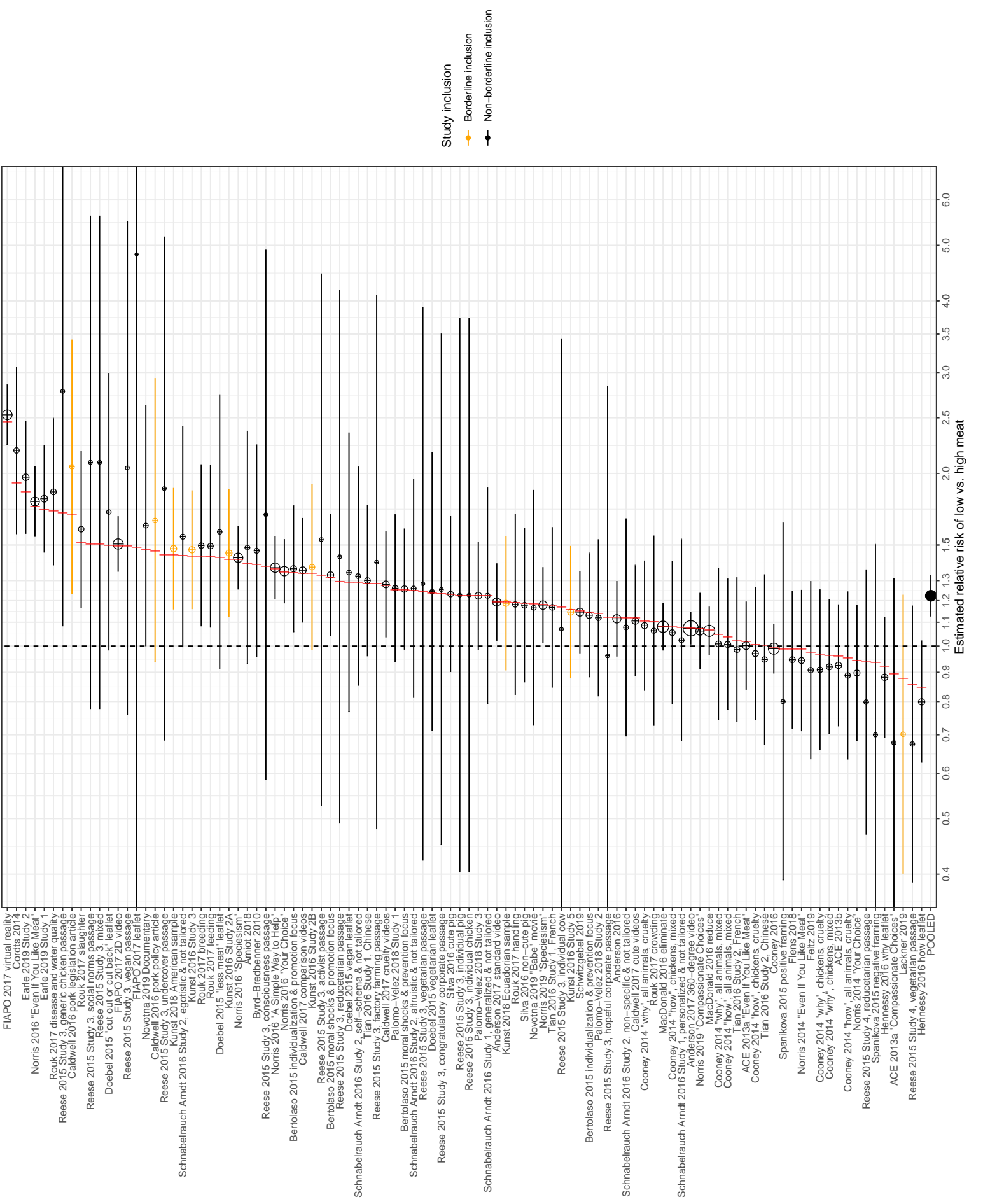

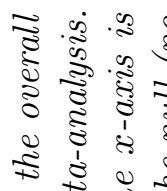

है है

के

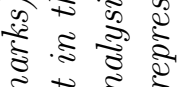

ह స्ञ है है

尔

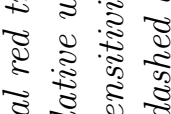

¿ु०

के का है

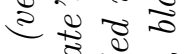

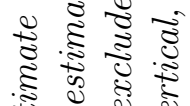

के

ग)

离

क के हैं

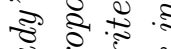

के है ड

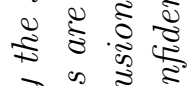

$\rightarrow \stackrel{0}{-3}$

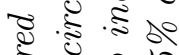

i.

కั

के के के के

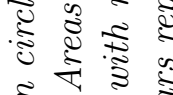

है

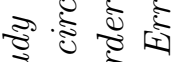

का ?

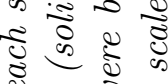

ป हु

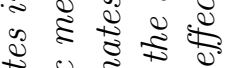

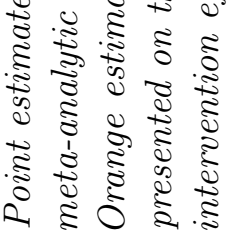

$\ddot{\sim}$

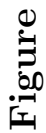




\section{Discussion}

\subsection{Summary of findings}

Despite authoritative calls for academic research and public policy regarding reducing meat consumption (Gardner et al., 2019; Godfray et al., 2018; Tilman and Clark, 2014; Scherer et al. 2019), the study of interventions to reduce meat consumption by appealing to animal welfare has largely remained outside the purview of academic research. Few, if any, attempts have been made to synthesize the evidence. In our systematic meta-analysis of this literature, we found that the body of evidence on this topic is well-developed both in terms of its size (100 studies reported in 34 articles) and in terms of the psychological sophistication of existing interventions (Section 1.1). The interventions appeared consistently beneficial at least in the short term and with outcomes primarily based on self-reported behavior or intended behavior, on average increasing by $22 \%$ an individual's probability of intending, self-reporting, or behaviorally demonstrating low versus high meat consumption.

Although it seemed plausible, a priori, that in some settings, animal welfare appeals could be in danger of backfiring (e.g., by being perceived as intrusively self-righteous and moralizing), our results suggested that this danger was rarely realized, as we estimated that the large majority of interventions $(83 \%)$ had true population effects in the beneficial rather than detrimental direction. This finding also suggests that the interventions were consistently effective across the culturally diverse samples we meta-analyzed, spanning at least 11 countries and four continents. However, it remains theoretically possible that even interventions with beneficial average effects could backfire for specific individuals (Rothgerber. 2020), a possibility that could be addressed in large studies by measuring individual-level effect modifiers or by assessing individually tailored interventions (Section 4.3).

These generally favorable results regarding animal welfare interventions do not imply, however, that appealing to animal welfare is necessarily more effective than, for example, appealing to concerns about health or the environment. To our knowledge, no quantitative 
meta-analysis of the latter types of interventions has been performed, precluding direct comparisons of evidence strength. Additionally, the literature on individuals' reported motivations for reducing meat consumption is somewhat mixed: a nationally representative survey in the United States (McCarthy and DeKoster, 2020) suggested that individuals who reported having reduced their meat consumption most often cited health as a major or minor motivation (90\% of subjects), though animal welfare was another important motivation $(65 \%$ of subjects). Another survey suggested that primary motivations were cost (51\% of subjects) and health (50\%), with concerns about animal welfare (12\%) and the environment (12\%) cited considerably less often (Neff et al., 2018).

We would speculate three potential explanations for these findings regarding the motivating role of animal welfare concerns relative to other concerns. First, these differences in reported motivations may simply reflect the prevalence of receiving information and interventions with different appeals, rather than differences in effectiveness between appeals. As described in the Introduction, the public is largely uninformed and even misinformed about farm animal welfare, reflecting successful efforts by the animal agriculture industry as well as individuals' deliberate avoidance of distressing information (Rothgerber, 2020; Cornish et al. 2016), whereas the public may be better informed about potential health consequences of excessive meat consumption. Second, if animal welfare interventions are indeed effective, this may be in part because they provide information that dissonates with many individuals' stated ethical values (Rothgerber, 2020; Cornish et al., 2016), perhaps leading these individuals to reduce meat consumption even if they would not do so out of concern for their own health or the environment. Third, food choices in general are shaped more by unconscious adherence to habit and situational cues than by conscious motivations van't Riet et al. 2011); we would therefore speculate that non-educational components of animal welfare interventions (such as leveraging the physical-moral disgust connection, invoking social norms, and giving implementation suggestions) might largely bypass conscious motivations in their influence on behavior. 
We investigated the associations of specific study and intervention characteristics with effectiveness. Critically, these meta-regressive estimates of effect modification based on covariates that vary across studies should not be interpreted as causal interactions due to potential confounding, as we further discuss below (Thompson and Higgins, 2002, VanderWeele and Knol, 2011). That is, these estimates represent the correlation between effect size and various characteristics of studies and interventions, not causation. We found that studies of interventions that recommended going vegan had estimated effects 31\% larger than interventions making no recommendation, and point estimates in studies whose interventions made intermediate recommendations to "go vegetarian" or "reduce consumption" heuristically suggested dose-response such that studies of interventions making more forceful recommendations (e.g., "go vegan" versus "reduce meat consumption") may have had larger effects on average. These preliminarily suggest that interventions that make more forceful recommendations do not seem to backfire and may in fact be more effective than subtler interventions. Studies measuring outcomes after a relatively long delay ( $\geq 7$ days) typically had smaller effect sizes by an estimated 19\%, though there was considerable statistical uncertainty due to the small number of studies with long follow-up times. Interventions' durations, their inclusion of text or visuals, and studies' percentages of male subjects did not appear meaningfully associated with effect size. Again, these estimates may not represent causal effects of different intervention designs; they could be confounded if, for example, more forceful interventions were typically used in studies of individuals who were already particularly motivated to reduce their meat consumption. Future research should conduct more head-to-head comparisons of interventions varying on these apparent effect modifiers, using randomization for those that can be directly manipulated (as in Cooney (2014); Macdonald et al. (2016)).

\subsection{Strengths and limitations}

The studies we meta-analyzed had some notable methodological strengths, including predominantly randomized designs, large sample sizes, and, especially among unpublished 
studies, a relatively high rate of data-sharing and preregistration. The studies also had limitations, including the potential for social desirability bias, the use of short lengths of follow-up (and typically high attrition when longer lengths of follow-up were used), and the use of self-reported rather than direct behavioral outcome measures; these issues were most apparent in the unpublished studies. Study designs appeared to be typically stronger with respect to exchangeability and external generalizability. However, a number of studies did appear susceptible to confounding due to a lack of randomization or differential attrition, and others may have had limited generalizability due to recruitment strategies that targeted highly motivated subjects or demographically restricted subjects (with samples tending to over-represent young North Americans, especially college undergraduates). Nevertheless, a number of sensitivity analyses, including those that included only studies at the lowest risk of bias by different criteria, had little effect on the results. Additionally, given the typically short lengths of follow-up, it is not clear whether interventions' effects were sustained long enough to meaningfully improve individual health, a point we discuss further in Section 4.3. On the other hand, inexpensive interventions such as leaflets or online videos could be deployed for a large number of individuals, potentially still producing meaningfully large environmental and animal welfare impacts in aggregate even if effects might have been short-lived for any given individual.

Regarding potential bias due to the literature's predominant reliance on self-reported outcome measures, such measures can introduce random noise if subjects estimate their consumption imperfectly. However, if this misreporting is, on average, no more or less severe for the intervention group than the control group, then typically intervention effect estimates would be either unbiased or biased toward, rather than away from, the null (Rothman et al. 2008). A more pernicious problem with self-report measures is their potential to also induce misreporting that is differential between the intervention and control group, which could potentially inflate estimates away from the null. Sensitivity analyses indicated that for the pooled point estimate to have been entirely attributable to such social 
desirability biases, interventions would have had to increase subjects' probability of reporting low meat consumption, independently of any effects the interventions may have had on actual consumption, by at least $22 \%$; and for social desirability bias of constant severity across studies to reduce to only $10 \%$ the percentage of true population effects stronger than $R R=1.1$, each intervention would have had to increase subjects' probability of reporting low meat consumption, independently of actual consumption, by at least $37 \%$. While it is clear that individuals in some cultures systematically under-report meat consumption in the absence of any meat-reducing intervention (Neff et al., 2018, Rothgerber, 2020), less is known about whether interventions reliably produce differential under-reporting of $22 \%$ to $37 \%$ in under-reporting above and beyond this baseline under-reporting.

Our meta-analysis contained mostly unpublished studies, including a large number conducted by animal advocacy nonprofits. These studies might in principle be more subject to influence by research funders than peer-reviewed academic studies. On the other hand, peer-reviewed studies might in principle be subject to stronger traditional publication bias favoring positive results. However, we observed no evidence to support either possibility. Instead, it appeared that the literature on animal welfare interventions overall did not show evidence of publication bias, and this held even when we considered only unpublished, or only published, studies (Section 3.4.1). Furthermore, the main meta-analytic means were similar in unpublished and published studies, and the former estimate was in fact somewhat smaller (Section 3.5). Additionally, unpublished studies showed a higher rate of preregistration, presumably limiting selective reporting. Selective reporting could also arise during our own processes of data extraction, analysis, or reporting, though we took considerable precautions to minimize this possibility, including publishing and preregistering the protocol in detail (Mathur et al. 2020), defining rules for data extraction that were as detailed and objective as possible, and documenting all post hoc analyses as such. 


\subsection{Recommendations for future research}

To further advance the field, one approach is to continue improving the interventions themselves, for example based on the potential effect modifiers identified above. Additionally, content could be tailored to individual subject characteristics, such as personality traits (Kaptein et al., 2012), individual receptivity to different types of recommendations or to graphic depictions of factory farming conditions, or disgust sensitivity (Schnall et al., 2008). Studies could also assess whether exposing subjects to the interventions repeatedly rather than only once might increase effects. However, given the psychological sophistication and apparent effectiveness of existing interventions and substantial body of existing research, we believe the field would be best served if future research were to first prioritize increasingly rigorous assessment of existing interventions.

To this end, we make three key methodological recommendations. Given financial constraints on study designs, we give concrete recommendations for study designs that may facilitate adopting these recommendations with relatively modest increases in cost, along with examples of existing studies that have demonstrated the feasibility of these designs. First, studies should use direct behavioral outcome measures of meat consumption rather than self-reports of behavior or of future intentions (Peacock, 2018), and the measures should be designed to minimize social desirability bias (Hebert et al., 1995). Self-reported dietary data can be subject to substantial measurement error even when collected via measures such as 24-hour recalls or food frequency questionnaires (Peacock, 2018, Freedman et al., 2014). Additionally, self-reported measures may exacerbate bias due to social desirability concerns: it is plausible that exposure to an animal welfare intervention could increase subjects' perceptions of social pressure to report lower meat consumption, and if outcomes are self-reported, subjects exposed to the intervention may report lower consumption than control subjects purely as an artifact of differential social desirability concerns. Some existing work has proposed or used behavioral measures based on purchase data from college dining 
halls or similar cafeteria settings (Haile et al. 2020; Jalil et al., 2019; Schwitzgebel et al., 2019, Garnett et al. 2019), meal choices at conferences (Hansen et al., 2019) or cafés (Sparkman and Walton, 2017, Anderson, 2020), or commercial marketing data providers (Peacock, 2018). Lab-based measurement of inexpensive clinical biomarkers of meat consumption, such as those obtained from hair samples, may be another cost-effective future avenue (Peacock, 2018 Cuparencu et al. 2019). Using measures such as purchase data and clinical biomarkers would also facilitate keeping subjects naïve to the outcome of interest and thus reduce social desirability bias.

Second, to determine how long interventions' effects last, studies should measure outcomes at longer follow-up times after the intervention (e.g., 1 month or more). When used to supplement pilot studies using shorter-term designs, such longitudinal follow-up would help identify whether some interventions' effects might be initially large but fade quickly, while other interventions' effects might be initially modest but sustained better. Additionally, longitudinal studies would enable principled assessment of psychological mediators, such as disgust or empathy, that underlie interventions' effects and effect maintenance (VanderWeele, 2015), which might help further refine interventions' content. Nevertheless, longer-term randomized studies may be prohibitively expensive and can introduce other methodological challenges, such as attrition. Introducing quasi-experimental designs, such as controlled interrupted time series or difference-in-difference methods (Bernal et al., 2017), to this field may facilitate following these recommendations. For example, quasi-experiments in which the intervention is deployed for a large group of subjects at the same time, rather than randomly assigned to individual subjects, might facilitate longitudinal collection of direct behavioral measures because these measures could be collected at the aggregate rather than individual level, and additionally without making subjects aware of that purpose of the study. Converging results from high-quality quasi-experiments and from randomized trials would strengthen the overall evidence base.

Third, outcome measures should be designed, when possible, to assess the numerical 
volume of meat eaten or purchased rather than only frequencies of consumption or purchase. Many existing studies measure meat consumption in terms of, for example, Likerttype items that categorize the number of weekly meals containing meat (e.g., "none", " $1-5$ meals", etc.) or in terms of reductions from one's previous consumption. When possible, using finer-grained absolute measures, such as the number of servings of poultry, beef, pork, lamb, fish, etc., would enable effect sizes to be translated into direct measures of societal impact, as described in Section 2.3. The typically coarse outcome measures in the current literature were not well-suited to conducting such analyses.

\subsection{Conclusion}

Our review and meta-analysis suggests that animal welfare interventions preliminarily appear effective, with meaningfully large effects, in these typically short-term studies of primarily selfreported outcomes. However, the existing literature does have some important methodological limitations. Although animal welfare interventions have received little academic attention to date, with the majority of studies reported in unpublished grey literature, our findings point to the importance of subjecting these promising interventions to increasingly rigorous and detailed empirical assessment. We recommend that interventions that appear effective in initial short-term studies with simple self-reported outcome measures - and our review has identified numerous existing strong candidates - should then be studied more rigorously using fine-grained, behavioral outcomes that minimize social desirability bias, and at longer time points following the intervention. Further developing the field of animal welfare interventions is a promising avenue for developing simple, effective interventions to reduce meat consumption, with potentially broad-reaching societal benefits. 


\section{TABLE LEGENDS}

Table 1: Basic characteristics of meta-analyzed studies and their interventions. Binary and categorical variables are reported as "count (percentage)". Continuous variables are reported as "median (first quartile, third quartile)". ${ }^{a}$ : The high proportion of missing data regarding studies' countries occurred because we coded country as missing for online studies (e.g., conducted on Amazon Mechanical Turk) that did not specifically state whether they used geographical restrictions when recruiting subjects.

Table 2: Study characteristics regarding design, analytic reproducibility, and risks of bias. Binary and categorical variables are reported as "count (percentage)". Continuous variables are reported as "median (first quartile, third quartile)". k: Number of studies in subset. RCT: randomized controlled trial. NRCT: non-randomized controlled trial. UCT: uncontrolled trial. ${ }^{a}$ : For studies in which missing data was unreported but the outcome was measured in the same session as the intervention, we coded missing data as 0. Details on the risk-of-bias categories are provided in the Supplement.

Table 3: Sensitivity analyses conducted on different groups of studies, with the overall estimate from the main analysis reported for comparison. Mean risk ratio: meta-analytic mean with 95\% confidence interval. p-value: for mean risk ratio versus null of 1 . $k$ : Number of studies in subset. $\widehat{\tau}$ : estimated standard deviation of true population effects on log-risk ratio scale. Final three columns: estimated percentage of true population effects stronger than various thresholds on risk ratio scale. Bracketed values are $95 \%$ confidence intervals for the percentage of effects stronger than a threshold, which were sometimes not estimable ("NA") when $\widehat{\tau}=0$ exactly or when the estimated proportion was very high.

Table 4: Meta-regressive estimates of effect modification by various study design and intervention characteristics (coarse model). Intercept: estimated mean risk ratio when all listed covariates are set to 0 . For binary covariates, estimates represent risk ratios for the increase in intervention effectiveness associated with a study's having, versus not having, the covariate. For the percentage of male subjects, the estimate represents the risk ratio for the increase in intervention effectiveness associated with a 10-percentage-point increase in males. For the average age, the estimate represents the risk ratio for the increase in intervention effectiveness associated with a 5-year increase in average subject age. CI: confidence interval. p-values are for the effect modification coefficients themselves, not for the subset of studies having the listed characteristic.

Table 5: Meta-regressive estimates of effect modification by various study design and intervention characteristics (fine-grained model), presented as in Table 4. 


\section{LIST OF ABBREVIATIONS}

$\mathrm{CI}=$ confidence interval; $\mathrm{RR}=$ risk ratio; $\mathrm{SSWS}=$ self-selected within-subjects

\section{DECLARATIONS}

Ethics approval and consent to participate

Not applicable.

Consent for publication

Not applicable.

Availability of data and materials

All data, materials, and analysis code required to reproduce all results are publicly available (https://osf.io/8zsw7/), with the exception of individual-subject data that we obtained by contacting authors and that cannot be made public at the authors' request. We have reported all deviations from the preregistration in the main text or Supplement.

\section{Competing interests}

JP is employed by The Humane League Labs, which is a program of, and currently fully funded through, The Humane League, a 501(c)(3) nonprofit organization that "exists to end the abuse of animals raised for food". The Humane League Labs is editorially independent from The Humane League, and any other potential funders, in reporting research results. The Humane League had no role in the design, conduct, or reporting of this research. All other authors have no conflicts of interest to report. 
Funding

This research was funded in part by The Pershing Square Fund for Research on the Foundations of Human Behavior. The funders had no role in the design, conduct, or reporting of this research.

\section{Authors' contributions}

MBM conceptualized the project. MBM, JP, DBR, and PAB designed the methodology with contributions from the other authors. DBR, JN, MBM, and JP reviewed articles and extracted qualitative data. MBM and JP extracted quantitative data. MBM conducted statistical analyses and wrote the manuscript, with all authors contributing critical revisions. All authors read and approved the final draft.

\section{Acknowledgments}

Jo Anderson (Research Director, Faunalytics) provided helpful suggestions on a draft of the protocol. Sophie Attwood (Senior Behavioral Scientist, Better Buying Lab) provided leads to grey literature. We are grateful to the many authors and organizations who made their data publicly available, who shared raw data with us upon request (including Jack Norris [Vegan Outreach] and Koushik Raghavan [Federation of Indian Animal Protection Organisations]), and who answered questions about their studies.

\section{REFERENCES}

Adriaanse, M. A., Vinkers, C. D., De Ridder, D. T., Hox, J. J., and De Wit, J. B. (2011). Do implementation intentions help to eat a healthy diet? A systematic review and meta-analysis of the empirical evidence. Appetite, 56(1):183-193. https://doi.org/10.1016/j.appet.2010.10.012.

Amiot, C. E., Boutros, G. E. H., Sukhanova, K., and Karelis, A. D. (2018). Testing a novel multicomponent intervention to reduce meat consumption in young men. PloS One, 13(10):e0204590. https://doi.org/10.1371/journal.pone.0204590. 
Anderson, E. C. and Barrett, L. F. (2016). Affective beliefs influence the experience of eating meat. PLoS One, 11(8):e0160424. https://doi.org/10.1371/journal.pone.0160424.

Anderson, J. (2017). An experimental investigation of the impact of video media on pork consumption. Technical report, Faunalytics. https://drive.google.com/file/ d/1ovZOxvJPKLGayAX-uCyGuZtJKCFcYOTP/view.

Anderson, J. (2020). "Reduce" or "Go Veg?" Effects on Meal Choice. Technical report, Faunalytics. https://faunalytics.org/reduce-or-go-veg/.

Arno, A. and Thomas, S. (2016). The efficacy of nudge theory strategies in influencing adult dietary behaviour: A systematic review and meta-analysis. BMC Public Health, 16(1):676. https://doi.org/10.1186/s12889-016-3272-x.

Bandura, A. (2002). Social foundations of thought and action: A social cognitive theory. In Marks, D. F., editor, The Health Psychology Reader, chapter 6, pages 94-106. https://doi.org/10.1017/S0813483900008238.

Bartlett, J. G., Gilbert, D. N., and Spellberg, B. (2013). Seven ways to preserve the miracle of antibiotics. Clinical Infectious Diseases, 56(10):1445-1450.

Bastian, B. and Loughnan, S. (2017). Resolving the meat-paradox: A motivational account of morally troublesome behavior and its maintenance. Personality and Social Psychology Review, 21(3):278-299. https://doi.org/10.1177/1088868316647562.

Bates, D., Mächler, M., Bolker, B., and Walker, S. (2015). Fitting linear mixed-effects models using lme4. Journal of Statistical Software, 67(1):1-48. https://doi.org/10.18637/jss.v067.i01

Benningstad, N. C. and Kunst, J. R. (2020). Dissociating meat from its animal origins: A systematic literature review. Appetite, 147. https://doi.org/10.1016/j.appet.2019.104554.

Bernal, J. L., Cummins, S., and Gasparrini, A. (2017). Interrupted time series regression for the evaluation of public health interventions: A tutorial. International Journal of Epidemiology, 46(1):348-355. https:/doi.org/10.1093/ije/dyw098.

Bertolaso, C. (2015). Investigating the effectiveness of message framing and regulatory fit in increasing positive animal attitude and reducing animal products consumption: A study for animal equality. Master's thesis, Universiteit van Amsterdam. https://scripties.uba.uva.nl/scriptie/570273.

Bianchi, F., Dorsel, C., Garnett, E., Aveyard, P., and Jebb, S. A. (2018a). Interventions targeting conscious determinants of human behaviour to reduce the demand for meat: A systematic review with qualitative comparative analysis. International Journal of Behavioral Nutrition and Physical Activity, 15(1):102. https://doi.org/10.1186/s12966-018-0729-6.

Bianchi, F., Garnett, E., Dorsel, C., Aveyard, P., and Jebb, S. A. (2018b). Restructuring physical micro-environments to reduce the demand for meat: A systematic review and qualitative comparative analysis. The Lancet Planetary Health, 2(9):e384-e397. https://doi.org/10.1016/S2542-5196(18)30188-8. 
Bonnet, C., Bouamra-Mechemache, Z., Réquillart, V., and Treich, N. (2020). Regulating meat consumption to improve health, the environment and animal welfare. Food Policy, page 101847. https://doi.org/10.1016/j.foodpol.2020.101847.

Brewer, N. T., Hall, M. G., Noar, S. M., Parada, H., Stein-Seroussi, A., Bach, L. E., Hanley, S., and Ribisl, K. M. (2016). Effect of pictorial cigarette pack warnings on changes in smoking behavior: a randomized clinical trial. JAMA Internal Medicine, 176(7):905-912. https://doi.org/10.1001/jamainternmed.2016.2621.

Bulow, E. (2016). rccmisc: Miscellaneous R Functions for Swedish Regional Cancer Centers. $\mathrm{R}$ package version 0.3.7. https://CRAN.R-project.org/package=rccmisc.

Byrd-Bredbenner, C., Grenci, A., and Quick, V. (2010). Effect of a television programme on nutrition cognitions and intended behaviours. Nutrition and Dietetics, 67(3):143-149. https://doi.org/10.1111/j.1747-0080.2010.01446.x.

Caldwell, K. (2016). Welfare reforms and meat consumption. Technical report, Mercy for Animals. https://mercyforanimals.org/welfare-reforms-survey.

Canty, A. and Ripley, B. D. (2020). boot: Bootstrap R (S-Plus) Functions. R package version $1.3-25$.

Chapman, H. A. and Anderson, A. K. (2013). Things rank and gross in nature: A review and synthesis of moral disgust. Psychological Bulletin, 139(2):300. https://doi.org/10.1037/a0030964.

Coburn, K. M. and Vevea, J. L. (2019). weightr: Estimating Weight-Function Models for Publication Bias. R package version 2.0.2. https://CRAN.R-project.org/package=weightr.

Cooney, N. (2014). What elements make a vegetarian leaflet more effective? Technical report, Humane League Labs. http://www.humaneleaguelabs.org/blog/2014-05-20-what-elementsmake-vegetarian-leaflet-more-effective/.

Cordts, A., Nitzko, S., and Spiller, A. (2014). Consumer response to negative information on meat consumption in Germany. International Food and Agribusiness Management Review, 17(Special Issue A):83-106. https:/doi.org/10.22004/ag.econ.164599.

Cornish, A., Raubenheimer, D., and McGreevy, P. (2016). What we know about the public's level of concern for farm animal welfare in food production in developed countries. Animals, 6(11):74. https://doi.org/10.3390/ani6110074.

Covidence Development Team (2019). Covidence Systematic Review Software. Veritas Health Innovation, Melbourne, Australia.

Crippa, A., Larsson, S. C., Discacciati, A., Wolk, A., and Orsini, N. (2018). Red and processed meat consumption and risk of bladder cancer: A dose-response meta-analysis of epidemiological studies. European Journal of Nutrition, 57(2):689-701. https://doi.org/10.1007/s00394016-1356-0. 
Cui, K., Liu, Y., Zhu, L., Mei, X., Jin, P., and Luo, Y. (2019). Association between intake of red and processed meat and the risk of heart failure: a meta-analysis. BMC Public Health, 19(1):354. https://doi.org/10.1186/s12889-019-6653-0.

Cuparencu, C., Praticó, G., Hemeryck, L. Y., Harsha, P. S. S., Noerman, S., Rombouts, C., Xi, M., Vanhaecke, L., Hanhineva, K., Brennan, L., et al. (2019). Biomarkers of meat and seafood intake: An extensive literature review. Genes $\& 3$ Nutrition, 14(1):1-30. https://doi.org/10.1186/s12263-019-0656-4.

da Silva, C. R. (2016). Am I too cute to eat? The effect of cuteness appeal towards the promotion of a more plant-based diet. Master's thesis, University Institute of Lisbon. http://hdl.handle.net/10071/12583.

Dahl, D. B., Scott, D., Roosen, C., Magnusson, A., and Swinton, J. (2019). xtable: Export Tables to LaTeX or HTML. R package version 1.8-4. https://CRAN.Rproject.org/package $=$ xtable.

Di Marco, M., Baker, M. L., Daszak, P., De Barro, P., Eskew, E. A., Godde, C. M., Harwood, T. D., Herrero, M., Hoskins, A. J., Johnson, E., et al. (2020). Opinion: Sustainable development must account for pandemic risk. Proceedings of the National Academy of Sciences, 117(8):3888-3892. https://doi.org/10.1073/pnas.2001655117.

Dowle, M. and Srinivasan, A. (2019). data.table: Extension of 'data.frame'. R package version 1.12.8. https://CRAN.R-project.org/package=data.table.

Earle, M., Hodson, G., Dhont, K., and MacInnis, C. (2019). Eating with our eyes (closed): Effects of visually associating animals with meat on antivegan/vegetarian attitudes and meat consumption willingness. Group Processes and Intergroup Relations, 22(6):1-18. https://doi.org/10.1177/1368430219861848.

Farvid, M. S., Stern, M. C., Norat, T., Sasazuki, S., Vineis, P., Weijenberg, M. P., Wolk, A., Wu, K., Stewart, B. W., and Cho, E. (2018). Consumption of red and processed meat and breast cancer incidence: A systematic review and meta-analysis of prospective studies. International Journal of Cancer, 143(11):2787-2799. https://doi.org/10.1002/ijc.31848.

Feinberg, M., Kovacheff, C., Teper, R., and Inbar, Y. (2019). Understanding the process of moralization: How eating meat becomes a moral issue. Journal of Personality and Social Psychology, 117:50-72. https://doi.org/10.1037/pspa0000149.

FIAPO (2017). Effectiveness assessment of outreach methods used by Federation of Indian Animal Protection Organisations (FIAPO). Technical report. New Delhi, India.

Fisher, Z. and Tipton, E. (2015). Robumeta: An R-package for robust variance estimation in meta-analysis. arXiv preprint arXiv:1503.02220. https://github.com/zackfisher/robumeta.

Fox, J. and Weisberg, S. (2019). An R Companion to Applied Regression. Sage, Thousand Oaks CA, third edition. https://socialsciences.mcmaster.ca/jfox/Books/Companion/. 
Freedman, L. S., Commins, J. M., Moler, J. E., Arab, L., Baer, D. J., Kipnis, V., Midthune, D., Moshfegh, A. J., Neuhouser, M. L., Prentice, R. L., et al. (2014). Pooled results from 5 validation studies of dietary self-report instruments using recovery biomarkers for energy and protein intake. American Journal of Epidemiology, 180(2):172-188. https://doi.org/10.1093/aje/kwu116.

Fretts, A. M., Follis, J. L., Nettleton, J. A., Lemaitre, R. N., Ngwa, J. S., Wojczynski, M. K., Kalafati, I. P., Varga, T. V., Frazier-Wood, A. C., Houston, D. K., et al. (2015). Consumption of meat is associated with higher fasting glucose and insulin concentrations regardless of glucose and insulin genetic risk scores: a meta-analysis of 50,345 caucasians. The American Journal of Clinical Nutrition, 102(5):1266-1278. https://doi.org/10.3945/ajcn.114.101238.

Gamer, M., Lemon, J., and <puspendra.pusp22@gmail.com>, I. F. P. S. (2019). irr: Various Coefficients of Interrater Reliability and Agreement. R package version 0.84.1. https://CRAN.R-project.org/package=irr.

Gardner, C. D., Hartle, J. C., Garrett, R. D., Offringa, L. C., and Wasserman, A. S. (2019). Maximizing the intersection of human health and the health of the environment with regard to the amount and type of protein produced and consumed in the United States. Nutrition Reviews, 77(4):197-215. https://doi.org/10.1093/nutrit/nuy073.

Garnett, E. E., Balmford, A., Sandbrook, C., Pilling, M. A., and Marteau, T. M. (2019). Impact of increasing vegetarian availability on meal selection and sales in cafeterias. Proceedings of the National Academy of Sciences, 116(42):20923-20929.

Gnagnarella, P., Caini, S., Maisonneuve, P., and Gandini, S. (2018). Carcinogenicity of high consumption of meat and lung cancer risk among nonsmokers: A comprehensive meta-analysis. Nutrition and Cancer, 70(1):1-13. https://doi.org/10.1080/01635581.2017.1374420.

Godfray, H. C. J., Aveyard, P., Garnett, T., Hall, J. W., Key, T. J., Lorimer, J., Pierrehumbert, R. T., Scarborough, P., Springmann, M., and Jebb, S. A. (2018). Meat consumption, health, and the environment. Science, 361(6399):eaam5324. https://doi.org/10.1126/science.aam5324.

Grattan, B. J. and Connolly-Schoonen, J. (2012). Addressing weight loss recidivism: A clinical focus on metabolic rate and the psychological aspects of obesity. ISRN Obesity, 2012. https://dx.doi.org/10.5402\%2F2012

Guasch-Ferré, M., Satija, A., Blondin, S. A., Janiszewski, M., Emlen, E., O'Connor, L. E., Campbell, W. W., Hu, F. B., Willett, W. C., and Stampfer, M. J. (2019). Metaanalysis of randomized controlled trials of red meat consumption in comparison with various comparison diets on cardiovascular risk factors. Circulation, 139(15):1828-1845. https://doi.org/10.1161/CIRCULATIONAHA.118.035225.

Haile, M., Jalil, A., and Tasoff, J. (2020). Changing hearts and plates: The effect of animal-advocacy pamphlets on meat consumption. Preprint retrieved from https://papers.ssrn.com/sol3/papers.cfm? abstract $_{i} d=3753878$. 
Hansen, P. G., Schilling, M., and Malthesen, M. S. (2019). Nudging healthy and sustainable food choices: three randomized controlled field experiments using a vegetarian lunch-default as a normative signal. Journal of Public Health.

Hebert, J. R., Clemow, L., Pbert, L., Ockene, I. S., and Ockene, J. K. (1995). Social desirability bias in dietary self-report may compromise the validity of dietary intake measures. International Journal of Epidemiology, 24(2):389-398. https://doi.org/10.1093/ije/24.2.389.

Hedges, L. V. (1992). Modeling publication selection effects in meta-analysis. Statistical Science, 7(2):246-255. https://doi.org/10.1214/ss/1177011364.

Hedges, L. V., Tipton, E., and Johnson, M. C. (2010). Robust variance estimation in metaregression with dependent effect size estimates. Research Synthesis Methods, 1(1):39-65. https://doi.org/10.1002/jrsm.5.

Hennessy, S. R. (2016). The impact of information on animal product consumption. Master's thesis, University of Illinois at Urbana-Champaign. https://www.ideals.illinois.edu/bitstream/handle/2142/92865/HENNESSY-THESIS2016. pdf? sequence $=1$ isAllowed $=\mathrm{y}$.

Higgs, S. (2015). Social norms and their influence on eating behaviours. Appetite, 86:38-44. https://doi.org/10.1016/j.appet.2014.10.021.

Hill, C. J., Bloom, H. S., Black, A. R., and Lipsey, M. W. (2008). Empirical benchmarks for interpreting effect sizes in research. Child Development Perspectives, 2(3):172-177. https://doi.org/10.1111/j.1750-8606.2008.00061.x.

Jalil, A., Tasoff, J., and Bustamante, A. (2019). Eating to save the planet: Evidence from a randomized controlled trial using individual-level food purchase data. Social Science Research Network (SSRN) Electronic Journal. https://dx.doi.org/10.2139/ssrn.3444642.

Jasper, J. M. and Poulsen, J. D. (1995). Recruiting strangers and friends: Moral shocks and social networks in animal rights and anti-nuclear protests. Social Problems, 42(4):493-512. https://doi.org/10.2307/3097043.

Jenni, K. and Loewenstein, G. (1997). Explaining the identifiable victim effect. Journal of Risk and Uncertainty, 14(3):235-257. https://doi.org/10.1023/A:1007740225484.

Jin, Z.-C., Zhou, X.-H., and He, J. (2015). Statistical methods for dealing with publication bias in meta-analysis. Statistics in Medicine, 34(2):343-360. https://doi.org/10.1002/sim.6342.

Kaptein, M., De Ruyter, B., Markopoulos, P., and Aarts, E. (2012). Adaptive persuasive systems: A study of tailored persuasive text messages to reduce snacking. ACM Transactions on Interactive Intelligent Systems (TiiS), 2(2):10. https://doi.org/10.1145/2209310.2209313.

Kim, K., Hyeon, J., Lee, S. A., Kwon, S. O., Lee, H., Keum, N., Lee, J.-K., and Park, S. M. (2017). Role of total, red, processed, and white meat consumption in stroke incidence and mortality: A systematic review and meta-analysis of prospective cohort studies. Journal of the American Heart Association, 6(9):e005983. https://doi.org/10.1161/JAHA.117.005983. 
Kim, Y. and Je, Y. (2018). Meat consumption and risk of metabolic syndrome: Results from the Korean population and a meta-analysis of observational studies. Nutrients, 10(4):390. https://doi.org/10.3390/nu10040390.

Knight, S. and Barnett, L. (2008). Justifying attitudes toward animal use: A qualitative study of people's views and beliefs. Anthrozoös, 21(1):31-42. https://doi.org/10.2752/089279308X274047.

Kuhn, M., Jackson, S., and Cimentada, J. (2020). corrr: Correlations in R. R package version 0.4.2. https://CRAN.R-project.org/package=corrr.

Kunst, J. R. and Haugestad, C. A. P. (2018). The effects of dissociation on willingness to eat meat are moderated by exposure to unprocessed meat: A cross-cultural demonstration. Appetite, 120:356-366. https://doi.org/10.1016/j.appet.2017.09.016.

Kunst, J. R. and Hohle, S. M. (2016). Meat eaters by dissociation: How we present, prepare and talk about meat increases willingness to eat meat by reducing empathy and disgust. Appetite, 105:758-774. https://psycnet.apa.org/doi/10.1016/j.appet.2017.09.016.

Lackner, Z. (2019). The effect of dissociation between meat and animal on the experience of ambivalence towards meat. Université de Grenoble Alpes.

Larsson, S. C. and Orsini, N. (2013). Red meat and processed meat consumption and all-cause mortality: A meta-analysis. American Journal of Epidemiology, 179(3):282-289. https://doi.org/10.1093/aje/kwt261.

Larsson, S. C. and Wolk, A. (2006). Meat consumption and risk of colorectal cancer: A meta-analysis of prospective studies. International Journal of Cancer, 119(11):2657-2664. https://doi.org/10.1001/jama.293.2.172.

Loughnan, S., Bastian, B., and Haslam, N. (2014). The psychology of eating animals. Current Directions in Psychological Science, 23(2):104-108. https://doi.org/10.1177\%2F0963721414525781.

Loughnan, S., Haslam, N., and Bastian, B. (2010). The role of meat consumption in the denial of moral status and mind to meat animals. Appetite, 55(1):156-159. https://doi.org/10.1016/j.appet.2010.05.043.

Lüdecke, D. (2020). sjstats: Statistical Functions for Regression Models (Version 0.18.0). https://CRAN.R-project.org/package=sjstats.

Macdonald, B., Caldwell, K., and Boese, G. (2016). The effects of 'reduce' and 'eliminate' appeals on individual meat consumption. Technical report, Reducetarian Foundation. https://osf.io/nxrx3/.

Machovina, B., Feeley, K. J., and Ripple, W. J. (2015). Biodiversity conservation: The key is reducing meat consumption. Science of the Total Environment, 536:419-431. https://doi.org/10.1016/j.scitotenv.2015.07.022. 
Marshall, B. and Levy, S. (2011). Food animals and antimicrobials: Impacts on human health. Clinical Microbiology Reviews, 24(4):718-733. https://doi.org/10.1128/cmr.00002-11.

Mathur, M. B., Robinson, T. N., Reichling, D. B., Gardner, C. D., Nadler, J., Bain, P. A., and Peacock, J. (2020). Reducing meat consumption by appealing to animal welfare: Protocol for a meta-analysis and theoretical review. Systematic Reviews, 9(1):1-8. https://doi.org/10.1186/s13643-019-1264-5.

Mathur, M. B. and VanderWeele, T. J. (2019). New metrics for meta-analyses of heterogeneous effects. Statistics in Medicine, pages 1336-1342. https://doi.org/10.1002/sim.8057.

Mathur, M. B. and VanderWeele, T. J. (2020a). PublicationBias: Sensitivity Analysis for Publication Bias in Meta-Analyses. R package version 2.1.0. https://CRAN.Rproject.org $/$ package $=$ PublicationBias.

Mathur, M. B. and VanderWeele, T. J. (2020b). Robust metrics and sensitivity analyses for meta-analyses of heterogeneous effects. Epidemiology, 9(1):1-8. https://doi.org/10.1097/ede.0000000000001180.

Mathur, M. B. and VanderWeele, T. J. (2020c). Sensitivity analysis for publication bias in meta-analyses. Journal of the Royal Statistical Society: Series C, 5(69):1091-1119.

Mathur, M. B., Wang, R., and VanderWeele, T. J. (2019). MetaUtility: Utility Functions for Conducting and Interpreting Meta-Analyses. R package version 2.1.0. https://CRAN.Rproject.org $/$ package $=$ MetaUtility.

McCarthy, J. and DeKoster, S. (2020). Nearly one in four in U.S. have cut back on eating meat. https://news.gallup.com/poll/282779/nearly-one-four-cut-back-eating-meat.aspx.

Neff, R. A., Edwards, D., Palmer, A., Ramsing, R., Righter, A., and Wolfson, J. (2018). Reducing meat consumption in the usa: a nationally representative survey of attitudes and behaviours. Public Health Nutrition, 21(10):1835-1844. https://doi.org/10.1017/S1368980017004190.

Neuwirth, E. (2014). RColorBrewer: ColorBrewer Palettes. $\mathrm{R}$ package version 1.1-2. https:/ CRAN.R-project.org/package $=$ RColorBrewer.

Norris, J. (2014). Booklet comparison study: Pay Per Read \#1. Technical report, Vegan Outreach. https://veganoutreach.org/pay-per-read-study-results/.

Norris, J. and Hannan, R. (2019). Leafleting and booklet effectiveness. Technical report, Vegan Outreach. https://veganoutreach.org/leafleting-and-booklet-effectiveness/.

Norris, J. and Roberts, E. (2016). Booklet comparison study - Pay Per Read \#2. Technical report, Vegan Outreach. https://veganoutreach.org/ppr-2016/.

Onwezen, M. C. and van der Weele, C. N. (2016). When indifference is ambivalence: Strategic ignorance about meat consumption. Food Quality and Preference, 52:96-105. https://doi.org/10.1016/j.foodqual.2016.04.001. 
Ooi, H., de Vries, A., and Microsoft (2020). checkpoint: Install Packages from Snapshots on the Checkpoint Server for Reproducibility. R package version 0.4.9. https://CRAN.Rproject.org $/$ package $=$ checkpoint.

Pan, A., Sun, Q., Bernstein, A. M., Schulze, M. B., Manson, J. E., Willett, W. C., and Hu, F. B. (2011). Red meat consumption and risk of type 2 diabetes: 3 cohorts of US adults and an updated meta-analysis. The American Journal of Clinical Nutrition, 94(4):1088-1096. https://doi.org/10.3945/ajcn.111.018978.

Peacock, J. (2018). Measuring change in diet for animal advocacy. Technical report, The Humane League Labs. https://thehumaneleague.org/article/E009R01-measuring-dietanimal-advocacy.

R Core Team (2020a). foreign: Read Data Stored by 'Minitab', 'S', 'SAS', 'SPSS', 'Stata', 'Systat', 'Weka', 'dBase', ... R package version 0.8-80. https://CRAN.Rproject.org $/$ package $=$ foreign .

R Core Team (2020b). R: A Language and Environment for Statistical Computing. $\mathrm{R}$ Foundation for Statistical Computing, Vienna, Austria. https://www.R-project.org/.

Reese, J. (2015). Testing the effectiveness of animal advocacy messages with Amazon Mechanical Turk. Technical report, The Sentience Institute. https://jacyreese.com/assets/downloads/Testing_the_Effectiveness.pdf.

Robinson, T. N. (2010). Save the world, prevent obesity: Piggybacking on existing social and ideological movements. Obesity, 18(S1):S17-S22. https://doi.org/10.1038/oby.2009.427.

Robinson, T. N. (2017). Stealth interventions for obesity: Strategies for behavioral, social, and policy changes. In Brownell, K. D. and Walsh, B. T., editors, Eating Disorders and Obesity: A Comprehensive Handbook, chapter 99, pages 609-613. Guilford Publications.

Robinson, T. N., Killen, J. D., Kraemer, H. C., Wilson, D. M., Matheson, D. M., Haskell, W. L., Pruitt, L. A., Powell, T. M., Owens, A., and Thompson, N. (2003). Dance and reducing television viewing to prevent weight gain in African-American girls: the Stanford GEMS pilot study. Ethnicity and Disease, 13(Suppl 1):S1-65. https://www.ethndis.org/priorsuparchives/ethn-13-s1-65.pdf.

Robinson, T. N., Matheson, D. M., Kraemer, H. C., Wilson, D. M., Obarzanek, E., Thompson, N. S., Alhassan, S., Spencer, T. R., Haydel, K. F., Fujimoto, M., et al. (2010). A randomized controlled trial of culturally tailored dance and reducing screen time to prevent weight gain in low-income African American girls: Stanford GEMS. Archives of Pediatrics and Adolescent Medicine, 164(11):995-1004. https://dx.doi.org/10.1001\%2Farchpediatrics.2010.197.

Rodriguez-Sanchez, F. (2018). grateful: Facilitate Citation of R Packages. R package version 0.0.2. https://github.com/Pakillo/grateful.

Rothgerber, H. (2020). Meat-related cognitive dissonance: A conceptual framework for understanding how meat eaters reduce negative arousal from eating animals. Appetite, 146:104511. https://doi.org/10.1016/j.appet.2019.104511. 
Rothman, K. J., Greenland, S., and Lash, T. L. (2008). Modern epidemiology. Lippincott Williams \& Wilkins.

Rouhani, M., Salehi-Abargouei, A., Surkan, P., and Azadbakht, L. (2014). Is there a relationship between red or processed meat intake and obesity? A systematic review and meta-analysis of observational studies. Obesity Reviews, 15(9):740-748. https://www.researchgate.net/deref/http

Rouk, S. (2017). Which farmed fish welfare issues does the public care most about? (Neutral messaging). Technical report, Mercy for Animals. https://mercyforanimals.org/which-fishwelfare-issue-generates-the-most.

Rozin, P. (1996). Towards a psychology of food and eating: From motivation to module to model to marker, morality, meaning, and metaphor. Current Directions in Psychological Science, 5(1):18-24. https://doi.org/10.1111\%2F1467-8721.ep10772690.

Rozin, P. and Fallon, A. (1980). The psychological categorization of foods and non-foods: A preliminary taxonomy of food rejections. Appetite, 1(3):193-201. https://doi.org/10.1016/S0195-6663(80)80027-4.

Rozin, P., Hormes, J. M., Faith, M. S., and Wansink, B. (2012). Is meat male? A quantitative multimethod framework to establish metaphoric relationships. Journal of Consumer Research, 39(3):629-643. https://doi.org/10.1086/664970.

Ruby, M. B. (2012). Vegetarianism. A blossoming field of study. Appetite, 58(1):141-150. https://doi.org/10.1016/j.appet.2011.09.019.

Sakadevan, K. and Nguyen, M.-L. (2017). Livestock production and its impact on nutrient pollution and greenhouse gas emissions. Advances in Agronomy, 141:147-184. https://doi.org/10.1016/bs.agron.2016.10.002.

Scherer, L., Behrens, P., and Tukker, A. (2019). Opportunity for a dietary winwin-win in nutrition, environment, and animal welfare. One Earth, 1(3):349-360. https://doi.org/10.1016/j.oneear.2019.10.020.

Schnall, S., Haidt, J., Clore, G. L., and Jordan, A. H. (2008). Disgust as embodied moral judgment. Personality and Social Psychology Bulletin, 34(8):1096-1109. https://dx.doi.org/10.1177\%2F0146167208317771.

Schultz, P. W., Nolan, J. M., Cialdini, R. B., Goldstein, N. J., and Griskevicius, V. (2007). The constructive, destructive, and reconstructive power of social norms. Psychological Science, 18(5):429-434. https://doi.org/10.1111/j.1467-9280.2007.01917.x.

Schwitzgebel, E., Cokelet, B., and Singer, P. (2019). Ethics classes can influence student behavior: Students purchase less meat after discussing arguments for vegetarianism. The Splintered Mind (Blog). http://schwitzsplinters.blogspot.com/2019/07/ethics-classes-caninfluence-student.html.

Singer, P. (1995). Animal Liberation. Random House. 
Sparkman, G. and Walton, G. M. (2017). Dynamic norms promote sustainable behavior, even if it is counternormative. Psychological Science, 28(11):1663-1674. https://doi.org/10.1177\%2F0956797617719950.

Sutton, A. J., Abrams, K. R., Jones, D. R., Jones, D. R., Sheldon, T. A., and Song, F. (2000). Methods for meta-analysis in medical research. Wiley Chichester.

Thompson, S. G. and Higgins, J. P. (2002). How should meta-regression analyses be undertaken and interpreted? Statistics in Medicine, 21(11):1559-1573. https://doi.org/10.1002/sim.1187.

Tian, Q., Hilton, D., and Becker, M. (2016). Confronting the meat paradox in different cultural contexts: Reactions among Chinese and French participants. Appetite, 96:187-194. https://doi.org/10.1016/j.appet.2015.09.009.

Tierney, N., Cook, D., McBain, M., and Fay, C. (2020). naniar: Data Structures, Summaries, and Visualisations for Missing Data. R package version 0.5.2. https://CRAN.Rproject.org $/$ package=naniar.

Tilman, D. and Clark, M. (2014). Global diets link environmental sustainability and human health. Nature, 515(7528):518-522. https://doi.org/10.1038/nature13959.

VanderWeele, T. J. (2015). Explanation in Causal Inference: Methods for Mediation and Interaction. Oxford University Press.

VanderWeele, T. J. (2017). On a square-root transformation of the odds ratio for a common outcome. Epidemiology, 28(6):e58-e60. https://doi.org/10.7326/0003-4819-154-10201105170-00008.

VanderWeele, T. J. (2019). Optimal approximate conversions of odds ratios and hazard ratios to risk ratios. Biometrics.

VanderWeele, T. J. and Knol, M. J. (2011). Interpretation of subgroup analyses in randomized trials: heterogeneity versus secondary interventions. Annals of Internal Medicine, 154(10):680-683. https://doi.org/10.7326/0003-4819-154-10-201105170-00008.

VanderWeele, T. J. and Li, Y. (2019). Simple sensitivity analysis for differential measurement error. American Journal of Epidemiology, 188(10):1823-1829. https://doi.org/10.1093/aje/kwz133.

van't Riet, J., Sijtsema, S. J., Dagevos, H., and De Bruijn, G.-J. (2011). The importance of habits in eating behaviour. an overview and recommendations for future research. Appetite, 57(3):585-596. https://doi.org/10.1016/j.appet.2011.07.010.

Västfjäll, D., Slovic, P., Mayorga, M., and Peters, E. (2014). Compassion fade: Affect and charity are greatest for a single child in need. PloS One, 9(6):e100115. https://doi.org/10.1371/journal.pone.0100115. 
Vegan Outreach (2018). Compassionate Choices. https://veganoutreach.org/wpcontent/uploads/2020/01/CC.pdf.

Vevea, J. L. and Hedges, L. V. (1995). A general linear model for estimating effect size in the presence of publication bias. Psychometrika, 60(3):419-435. https://doi.org/10.1007/BF02294384.

Viechtbauer, W. (2010). Conducting meta-analyses in R with the metafor package. Journal of Statistical Software, 36(3):1-48. https://www.jstatsoft.org/v36/i03/.

Walton, G. M. (2014). The new science of wise psychological interventions. Current Directions in Psychological Science, 23(1):73-82. https://doi.org/10.1177\%2F0963721413512856.

Wang, C.-C. and Lee, W.-C. (2019). A simple method to estimate prediction intervals and predictive distributions: Summarizing meta-analyses beyond means and confidence intervals. Research Synthesis Methods, 10(2):255-266. https://doi.org/10.1002/jrsm.1345.

Wang, X., Lin, X., Ouyang, Y. Y., Liu, J., Zhao, G., Pan, A., and Hu, F. B. (2016). Red and processed meat consumption and mortality: dose-response metaanalysis of prospective cohort studies. Public Health Nutrition, 19(5):893-905. https://doi.org/10.1017/s1368980015002062.

Weintraub, D. L., Tirumalai, E. C., Haydel, K. F., Fujimoto, M., Fulton, J. E., and Robinson, T. N. (2008). Team sports for overweight children: The Stanford Sports to Prevent Obesity Randomized Trial (SPORT). Archives of Pediatrics and Adolescent Medicine, 162(3):232-237. https://doi.org/10.1001/archpediatrics.2007.43.

Wickham, H. (2011a). The split-apply-combine strategy for data analysis. Journal of Statistical Software, 40(1):1-29. http://www.jstatsoft.org/v40/i01/.

Wickham, H. (2011b). testthat: Get started with testing. The $R$ Journal, 3:5-10. https://doi.org/10.32614/RJ-2011-002.

Wickham, H. (2016). ggplot2: Elegant Graphics for Data Analysis. Springer-Verlag New York. https://ggplot2.tidyverse.org.

Wickham, H. (2019). stringr: Simple, Consistent Wrappers for Common String Operations. $\mathrm{R}$ package version 1.4.0. https://CRAN.R-project.org/package=stringr.

Wickham, H., Averick, M., Bryan, J., Chang, W., McGowan, L. D., François, R., Grolemund, G., Hayes, A., Henry, L., Hester, J., Kuhn, M., Pedersen, T. L., Miller, E., Bache, S. M., Müller, K., Ooms, J., Robinson, D., Seidel, D. P., Spinu, V., Takahashi, K., Vaughan, D., Wilke, C., Woo, K., and Yutani, H. (2019). Welcome to the tidyverse. Journal of Open Source Software, 4(43):1686. https://doi.org/10.21105/joss.01686.

Wickham, H. and Bryan, J. (2019). readxl: Read Excel Files. R package version 1.3.1. https://CRAN.R-project.org/package=readxl. 
Wickham, H., François, R., Henry, L., and Müller, K. (2020). dplyr: A Grammar of Data Manipulation. R package version 1.0.0. https://CRAN.R-project.org/package=dplyr.

Wickham, H. and Henry, L. (2020). tidyr: Tidy Messy Data. R package version 1.1.0. https://CRAN.R-project.org/package=tidyr.

Wolak, M. E., Fairbairn, D. J., and Paulsen, Y. R. (2012). Guidelines for estimating repeatability. Methods in Ecology and Evolution, 3(1):129-137. https://doi.org/10.1111/j.2041210X.2011.00125.x.

Wrenn, C. L. (2013). Resonance of moral shocks in abolitionist animal rights advocacy: Overcoming contextual constraints. Society \& Animals, 21(4):379-394. https://doi.org/10.1163/15685306-12341271.

Yoshida, K. (2020). tableone: Create 'Table 1' to Describe Baseline Characteristics. R package version 0.11.1. https://CRAN.R-project.org/package=tableone.

Zeileis, A. (2004). Econometric computing with HC and HAC covariance matrix estimators. Journal of Statistical Software, 11(10):1-17. http://dx.doi.org/10.18637/jss.v011.i10.

Zhang, Y. and Zhang, D. Z. (2018). Red meat, poultry, and egg consumption with the risk of hypertension: A meta-analysis of prospective cohort studies. Journal of Human Hypertension, 32(7):507. https://doi.org/10.1038/s41371-018-0068-8. 


\begin{tabular}{|c|c|}
\hline Characteristic & $\begin{array}{c}\text { Number of studies }(\%) \\
\text { or median }(\mathrm{Q} 1, \mathrm{Q} 3)\end{array}$ \\
\hline \multicolumn{2}{|l|}{ Country } \\
\hline Canada & $1(1 \%)$ \\
\hline China & $2(2 \%)$ \\
\hline Czech Republic & $2(2 \%)$ \\
\hline Ecuador & $1(1 \%)$ \\
\hline France & $3(3 \%)$ \\
\hline Germany & $1(1 \%)$ \\
\hline India & $3(3 \%)$ \\
\hline Netherlands & $1(1 \%)$ \\
\hline Portugal & $2(2 \%)$ \\
\hline Scotland & $2(2 \%)$ \\
\hline USA & $37(37 \%)$ \\
\hline USA, Canada & $2(2 \%)$ \\
\hline Not reported $^{\mathrm{a}}$ & $43(43 \%)$ \\
\hline Percent male subjects & $43(36.1,51.7)$ \\
\hline Not reported & $4(4 \%)$ \\
\hline Average age (years) & $33.7(21.9,35.4)$ \\
\hline Not reported & $9(9 \%)$ \\
\hline \multicolumn{2}{|l|}{ Student recruitment } \\
\hline Not undergraduates & $77(77 \%)$ \\
\hline General undergraduates & $10(10 \%)$ \\
\hline Social sciences undergraduates & $3(3 \%)$ \\
\hline Mixed & $10(10 \%)$ \\
\hline \multicolumn{2}{|l|}{ Intervention had text } \\
\hline Yes & $83(83 \%)$ \\
\hline No & $14(14 \%)$ \\
\hline Not reported & $3(3 \%)$ \\
\hline \multicolumn{2}{|l|}{ Intervention had visuals } \\
\hline Yes & $62(62 \%)$ \\
\hline No & $36(36 \%)$ \\
\hline Not reported & $2(2 \%)$ \\
\hline \multicolumn{2}{|l|}{ Intervention had graphic content } \\
\hline Yes & $61(61 \%)$ \\
\hline No & $37(37 \%)$ \\
\hline Not reported & $2(2 \%)$ \\
\hline \multicolumn{2}{|l|}{ Intervention used mind attribution } \\
\hline Yes & $65(65 \%)$ \\
\hline No & $28(28 \%)$ \\
\hline Not reported & $7(7 \%)$ \\
\hline \multicolumn{2}{|l|}{ Intervention used social norms } \\
\hline Yes & $29(29 \%)$ \\
\hline No & $62(62 \%)$ \\
\hline Not reported & $9(9 \%)$ \\
\hline $\begin{array}{l}\text { Intervention identified } \\
\text { a named victim }\end{array}$ & \\
\hline Yes & $21(21 \%)$ \\
\hline No & $70(70 \%)$ \\
\hline Not reported & $9(9 \%)$ \\
\hline Intervention depicted pets & \\
\hline Yes & $32(32 \%)$ \\
\hline No & $60(60 \%)$ \\
\hline Not reported & $8(8 \%)$ \\
\hline Intervention gave & \\
\hline implementation suggestions & \\
\hline Yes & $29(29 \%)$ \\
\hline No & $61(61 \%)$ \\
\hline Not reported & $10(10 \%)$ \\
\hline Intervention described animal welfare & \\
\hline Yes & $52(52 \%)$ \\
\hline No & $44(44 \%)$ \\
\hline Not reported & $4(4 \%)$ \\
\hline Intervention was personally tailored & \\
\hline Yes & $2(2 \%)$ \\
\hline No & $98(98 \%)$ \\
\hline Not reported & $0(0 \%)$ \\
\hline Intervention's recommendation & \\
\hline No recommendation & $43(43 \%)$ \\
\hline "Reduce consumption" & $13(13 \%)$ \\
\hline "Go vegetarian" & $9(9 \%)$ \\
\hline "Go vegan" & $7(7 \%)$ \\
\hline Mixed recommendation & $24(24 \%)$ \\
\hline Not reported & $4(4 \%)$ \\
\hline Intervention's duration (minutes) & $1.5(1,5.88)$ \\
\hline Not reported & $2(2 \%)$ \\
\hline Outcome category & \\
\hline Consumption & $96(96 \%)$ \\
\hline Purchase & $4(4 \%)$ \\
\hline Length of follow-up (days) & $0(0,32.5)$ \\
\hline Not reported & $1(1 \%)$ \\
\hline Source of statistics & \\
\hline Data from author & $19(19 \%)$ \\
\hline Paper & $28(28 \%)$ \\
\hline Public data & $53(53 \%)$ \\
\hline
\end{tabular}


Table 2

\begin{tabular}{|c|c|c|c|}
\hline Characteristic & $\begin{array}{c}\text { All studies } \\
(\mathbf{k}=\mathbf{1 0 0})\end{array}$ & $\begin{array}{c}\text { Published studies } \\
(k=17)\end{array}$ & $\begin{array}{c}\text { Unpublished studies } \\
(\mathrm{k}=\mathbf{8 3})\end{array}$ \\
\hline \multicolumn{4}{|l|}{ Design } \\
\hline Between-subjects RCT & $72(72 \%)$ & $16(94 \%)$ & $56(67 \%)$ \\
\hline Cluster RCT & $2(2 \%)$ & $0(0 \%)$ & $2(2 \%)$ \\
\hline Between-subjects NRCT & $21(21 \%)$ & $0(0 \%)$ & $21(25 \%)$ \\
\hline \multicolumn{4}{|l|}{ Outcome measurement } \\
\hline Direct behavioral measure & $2(2 \%)$ & $1(6 \%)$ & $1(1 \%)$ \\
\hline Self-reported past behavior & $41(41 \%)$ & $1(6 \%)$ & $40(48 \%)$ \\
\hline Intended future behavior & $57(57 \%)$ & $15(88 \%)$ & $42(51 \%)$ \\
\hline Low risk of bias & $52(52 \%)$ & $16(94 \%)$ & $36(43 \%)$ \\
\hline Medium risk of bias & $9(9 \%)$ & $1(6 \%)$ & $8(10 \%)$ \\
\hline High risk of bias & $29(29 \%)$ & $0(0 \%)$ & $29(35 \%)$ \\
\hline Unclear & $10(10 \%)$ & $0(0 \%)$ & $10(12 \%)$ \\
\hline \multicolumn{4}{|c|}{ Avoidance of social desirability bias } \\
\hline Low risk of bias & $13(13 \%)$ & $6(35 \%)$ & $7(8 \%)$ \\
\hline Medium risk of bias & $14(14 \%)$ & $5(29 \%)$ & $9(11 \%)$ \\
\hline High risk of bias & $68(68 \%)$ & $6(35 \%)$ & $62(75 \%)$ \\
\hline Unclear & $5(5 \%)$ & $0(0 \%)$ & $5(6 \%)$ \\
\hline \multicolumn{4}{|l|}{ Preregistered } \\
\hline Yes & $25(25 \%)$ & $2(12 \%)$ & $23(28 \%)$ \\
\hline No & $75(75 \%)$ & $15(88 \%)$ & $60(72 \%)$ \\
\hline \multicolumn{4}{|l|}{ Public data } \\
\hline Yes & $53(53 \%)$ & $5(29 \%)$ & $48(58 \%)$ \\
\hline No & $47(47 \%)$ & $12(71 \%)$ & $35(42 \%)$ \\
\hline \multicolumn{4}{|l|}{ Public code } \\
\hline Yes & $22(22 \%)$ & $4(24 \%)$ & $18(22 \%)$ \\
\hline No & $78(78 \%)$ & $13(76 \%)$ & $65(78 \%)$ \\
\hline \multicolumn{4}{|l|}{ Overall lowest risk of bias } \\
\hline Yes & $12(12 \%)$ & $9(53 \%)$ & $3(4 \%)$ \\
\hline No & $88(88 \%)$ & $8(47 \%)$ & $80(96 \%)$ \\
\hline
\end{tabular}


Table 3

\begin{tabular}{|c|c|c|c|c|c|c|c|}
\hline Studies analyzed & $\mathbf{k}$ & Mean risk ratio & p-value & $\hat{\tau}$ & $\%$ above 1 & $\%$ above 1.1 & $\%$ above 1.2 \\
\hline Main analysis & 100 & $1.22[1.13,1.33]$ & $<0.0001$ & 0.12 & $83[72,91]$ & $71[59,80]$ & $53[38,64]$ \\
\hline $\begin{array}{l}\text { Excluding borderline-eligible } \\
\text { studies }\end{array}$ & 91 & $1.21[1.11,1.33]$ & 0.0003 & 0.12 & $82[69,91]$ & $68[55,77]$ & $52[35,63]$ \\
\hline Excluding composite interventions & 52 & $1.29[1.18,1.40]$ & $<0.0001$ & 0.11 & $98[\mathrm{NA}, \mathrm{NA}]$ & $87[71,94]$ & $71[38,83]$ \\
\hline Studies at lowest risk of bias & 12 & $1.3[0.98,1.72]$ & 0.06 & 0.21 & $92[\mathrm{NA}, \mathrm{NA}]$ & $75[0,92]$ & $58[8,83]$ \\
\hline $\begin{array}{l}\text { Excluding studies measuring } \\
\text { intended behavior }\end{array}$ & 43 & $1.11[0.98,1.26]$ & 0.08 & 0.14 & $70[47,88]$ & $47[26,60]$ & $30[9,49]$ \\
\hline Randomized studies & 75 & $1.24[1.14,1.34]$ & $<0.0001$ & 0.15 & $93[79,99]$ & $80[66,88]$ & $61[36,77]$ \\
\hline $\begin{array}{l}\text { Preregistered studies with } \\
\text { open data }\end{array}$ & 21 & $1.09[0.99,1.19]$ & 0.06 & 0 & $100[\mathrm{NA}, \mathrm{NA}]$ & $0[\mathrm{NA}, \mathrm{NA}]$ & $0[\mathrm{NA}, \mathrm{NA}]$ \\
\hline Published studies & 17 & $1.35[1.09,1.67]$ & 0.02 & 0.16 & $100[\mathrm{NA}, \mathrm{NA}]$ & 88 [NA, NA] & $71[0,94]$ \\
\hline Unpublished studies & 83 & $1.19[1.08,1.32]$ & 0.001 & 0.13 & $80[65,89]$ & $66[52,77]$ & $51[34,64]$ \\
\hline Excluding one extreme estimate & 99 & $1.22[1.13,1.33]$ & $<0.0001$ & 0.1 & $83[71,91]$ & $71[59,80]$ & $53[36,64]$ \\
\hline Including SSWS studies & 108 & $1.31[1.19,1.44]$ & $<0.0001$ & 0 & $81[71,87]$ & $69[56,76]$ & $56[44,65]$ \\
\hline
\end{tabular}


Table 4

\begin{tabular}{lcc}
\hline \multicolumn{1}{c}{ Coefficient } & Effect modification RR [95\% CI] & p-value \\
\hline Intercept & $1.11[0.66,1.86]$ & 0.66 \\
Intervention had text & $0.85[0.68,1.06]$ & 0.12 \\
Intervention had visuals & $0.96[0.81,1.13]$ & 0.57 \\
Intervention had graphic content & $1.10[0.97,1.24]$ & 0.13 \\
Intervention's recommendation & & Ref. \\
$\quad$ No recommendation & Ref. & 0.98 \\
$\quad$ "Reduce consumption" & $1.00[0.77,1.31]$ & 0.81 \\
$\quad$ "Go vegetarian" & $1.03[0.78,1.36]$ & 0.03 \\
$\quad$ Mixed recommendation & $1.31[1.06,1.62]$ & 0.94 \\
Intervention duration $>\mathbf{5}$ minutes & $0.99[0.83,1.19]$ & 0.70 \\
Follow-up length at least 7 days & $1.03[0.86,1.24]$ & 0.03 \\
Percentage male subjects & $0.81[0.68,0.97]$ & \\
(10-pt increase) & & 0.88 \\
Average subject age (5-year & $1.00[0.95,1.06]$ & \\
increase) & & 0.15 \\
\hline
\end{tabular}


Table 5

\begin{tabular}{lcc|}
\hline \multicolumn{1}{c}{ Coefficient } & Effect modification RR [95\% CI] & p-value \\
\hline Intercept & $0.87[0.51,1.46]$ & 0.56 \\
Intervention used mind attribution & $1.00[0.74,1.33]$ & 0.97 \\
Intervention used social norms & $1.03[0.64,1.65]$ & 0.88 \\
Intervention identified victim & $0.94[0.65,1.35]$ & 0.68 \\
Intervention depicted pets & $1.11[0.70,1.78]$ & 0.47 \\
Intervention used implementation & & 0.22 \\
suggestions & $1.24[0.85,1.80]$ & 0.61 \\
Intervention had visuals & $1.04[0.87,1.25]$ & 0.45 \\
Intervention had graphic content & $1.07[0.88,1.29]$ & \\
Intervention's recommendation & & $R e f$. \\
$\quad$ No recommendation & & 0.33 \\
$\quad$ "Reduce consumption" & & 0.55 \\
$\quad$ "Go vegetarian" & $0.89[0.68,1.17]$ & 0.55 \\
$\quad$ "Go vegan" & $0.91[0.63,1.31]$ & 0.29 \\
$\quad$ Intervention duration $>\mathbf{5}$ minutes & $1.25[0.78,1.99]$ & 0.14 \\
Follow-up length at least 7 days & $0.78[0.54,1.12]$ & 0.81 \\
\hline Average subject age (5-year increase) & $0.96[0.66,1.40]$ & 0.15 \\
\hline
\end{tabular}




\title{
Supplement: Interventions to Reduce Meat Consumption by Appealing to Animal Welfare: Meta-Analysis and Evidence-Based Recommendations
}

\author{
CONTENTS
}

\begin{tabular}{lll}
\hline 1 & Supplementary methods & 2
\end{tabular}

1.1 Details of search process . . . . . . . . . . . . . . . 2

1.1.1 Methods for searching peer-reviewed literature . . . . . . . . . . . . . 2

1.1 .2 Methods for searching grey literature . . . . . . . . . . . . . . . . . 4

1.2 Details of data extraction . . . . . . . . . . . . . . . . . . . . 6

1.2.1 Qualitative data extraction . . . . . . . . . . . . . 6

1.2 .2 Quantitative data extraction . . . . . . . . . . . . . . . 12

1.3 Details of sensitivity analysis for social desirability bias . . . . . . . . . . . 13

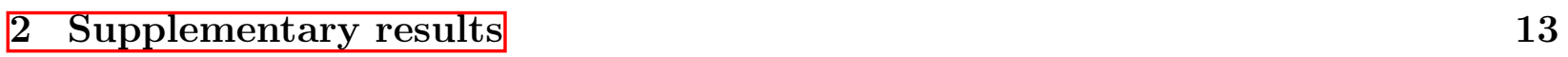

2.1 Reference lists of included articles . . . . . . . . . . . . . . . . 13

$2.1 .1 \quad$ Articles included in main analysis . . . . . . . . . . . . . . . . . . 13

$2.1 .2 \quad$ Articles reporting on SSWS studies . . . . . . . . . . . . . . . . 16

2.2 Supplementary tables . . . . . . . . . . . . . . . . . . . . . 17

2.3 Supplementary figures . . . . . . . . . . . . . . . . . . 20

3 Changes and deviations from preregistered protocol 24 


\section{SuPPlEMENTARY METHODS}

\subsection{Details of search process}

\subsubsection{Methods for searching peer-reviewed literature}

The search terms used for each database were as follows.

MEDLINE (Ovid) (animal welfare/ OR emotions/ OR disgust/ OR fear/ OR guilt/ OR empathy/ OR morals/ OR (emotion* OR affective OR disgust* OR fear OR guilt* OR empath* OR suffer* OR moral* OR ethic* OR humane* OR anthropomorph* OR belief* OR cognitive dissonan* OR meat paradox).ab,ti OR (animal* adj6 (welfare OR rights OR wellbeing OR well being OR cruel* OR abuse OR mistreat*)).ab,ti) AND ((meat* OR animal product* OR beef OR veal OR lamb OR pork OR poultry OR chicken OR turkey OR pig OR cow OR sheep) adj6 (consum* OR eat? OR eating OR ate OR intak* OR purchas* OR buy* OR demand* OR choos* OR choice* OR avoid* OR prefer*)).ab,ti

Embase (Elsevier) ('animal welfare'/exp OR 'emotion'/de OR 'disgust'/de OR 'fear'/de OR 'guilt'/de OR 'empathy'/exp OR 'morality'/exp OR (emotion* OR affective OR disgust* OR fear OR guilt* OR empath* OR suffer* OR moral* OR ethic* OR humane* OR anthropomorph* OR belief* OR 'cognitive dissonan*' OR 'meat paradox'):ab,ti OR (animal* NEAR/6 (welfare OR rights OR wellbeing OR 'well being' OR cruel* OR abuse OR mistreat*)):ab,ti) AND ('meat consumption'/ exp OR ((meat* OR 'animal product*' OR beef OR veal OR lamb OR pork OR poultry OR chicken OR turkey OR pig OR cow OR sheep) NEAR/6 (consum* OR eat OR eats OR eating OR ate OR intak* OR purchas* OR buy* OR demand* OR choos* OR choice* OR avoid* OR prefer*)):ab,ti)

Web of Science (Clarivate Analytics) $\mathrm{TS}=($ "emotion*" OR "affective" OR "disgust*" OR "fear" OR "guilt*" OR "empath*" OR "suffer*" OR "moral*" OR "ethic*" OR "humane*" OR "anthropomorph*" OR "belief*" OR "cognitive dissonan*" OR "meat paradox" OR ("animal*" NEAR/6 ("welfare" OR "rights" OR "wellbeing" OR "well being" OR "cruel*" OR "abuse" OR "mistreat*"))) AND TS=(("meat*" OR "animal product*" OR "beef" OR "veal" OR "lamb" OR "pork" OR "poultry" OR "chicken" OR "turkey" OR "pig" OR "cow" OR "sheep") NEAR/6 ("consum*" OR "eat" OR "eats" OR "eating" OR "ate" OR "intak*" OR "purchas*" OR "buy*" OR "demand*" OR "choos*" OR "choice*" OR "avoid*" OR "prefer*"))

PsycINFO (EBSCO) (DE ("Animal Welfare" OR "Animal Cruelty" OR "Animal Rights" OR "Emotional Content" OR "Emotional Responses" OR "Emotions" OR "Disgust" OR 
"Fear" OR "Guilt" OR "Empathy" OR "Morality" OR "Cognitive Dissonance") OR TI (emotion* OR affective OR disgust* OR fear OR guilt* OR empath* OR suffer* OR moral* OR ethic* OR humane* OR anthropomorph* OR belief* OR "cognitive dissonan*" OR "meat paradox" OR (animal* N6 (welfare OR rights OR wellbeing OR "well being" OR

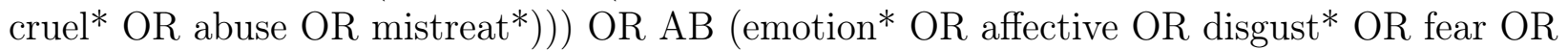
guilt* OR empath* OR suffer* OR moral* OR ethic* OR humane* OR anthropomorph* OR belief* OR "cognitive dissonan*" OR "meat paradox" OR (animal* N6 (welfare OR rights OR wellbeing OR "well being" OR cruel* OR abuse OR mistreat*)))) AND (TI ((meat* OR "animal product*" OR beef OR veal OR lamb OR pork OR poultry OR chicken OR turkey OR pig OR cow OR sheep) N6 (consum* OR eat OR eats OR eating OR ate OR intak* OR purchas* OR buy* OR demand* OR choos* OR choice* OR avoid* OR prefer*)) OR AB ((meat* OR "animal product*" OR beef OR veal OR lamb OR pork OR poultry OR chicken OR turkey OR pig OR cow OR sheep) N6 (consum* OR eat OR eats OR eating OR ate OR intak* OR purchas* OR buy* OR demand* OR choos* OR choice* OR avoid* OR prefer*)))

CAB Abstracts (Clarivate Analytics) $\mathrm{TS}=($ "emotion*" OR "affective" OR "disgust*" OR "fear" OR "guilt*" OR "empath*" OR "suffer*" OR "moral*" OR "ethic*" OR "humane*" OR "anthropomorph*" OR "belief*" OR "cognitive dissonan*" OR "meat paradox" OR ("animal*" NEAR/6 ("welfare" OR "rights" OR "wellbeing" OR "well being" OR "cruel*" OR "abuse" OR "mistreat*"))) AND TS=(("meat*" OR "animal product*" OR "beef" OR "veal" OR "lamb" OR "pork" OR "poultry" OR "chicken" OR "turkey" OR "pig" OR "cow" OR "sheep") NEAR/6 ("consum*" OR "eat" OR "eats" OR "eating" OR "ate" OR "intak*" OR "purchas*" OR "buy*" OR "demand*" OR "choos*" OR "choice*" OR "avoid*" OR "prefer*"))

Sociological Abstracts (ProQuest) (SU.EXACT("Animal Human Relations" OR "Emotions" OR "Guilt" OR "Fear" OR "Empathy" OR "Morality" OR "Moral Judgement" OR "Cognitive Disonance") OR TI(emotion* OR affective OR disgust* OR fear OR guilt* OR empath* OR suffer* OR moral* OR ethic* OR humane* OR anthropomorph* OR belief* OR "cognitive dissonan*" OR "meat paradox" OR (animal* NEAR/6 (welfare OR rights OR wellbeing OR "well being" OR cruel* OR abuse OR mistreat*))) OR AB(emotion* OR affective OR disgust* OR fear OR guilt* OR empath* OR suffer* OR moral* OR ethic* OR humane* OR anthropomorph* OR belief* OR "cognitive dissonan*" OR "meat paradox" OR (animal* NEAR/6 (welfare OR rights OR wellbeing OR "well being" OR cruel* OR abuse OR mistreat*) $)$ ) AND ((meat* OR "animal product*" OR beef OR veal OR lamb OR pork OR poultry OR chicken OR turkey OR pig OR cow OR sheep) NEAR/6 (consum* OR eat OR eats OR eating OR ate OR intak* OR purchas* OR buy* OR demand* OR choos* OR choice* OR avoid* OR prefer*))

ProQuest Dissertations and Theses (ProQuest) noft $(($ meat* OR "animal product*" OR beef OR veal OR lamb OR pork OR poultry OR chicken OR turkey OR pig OR cow OR sheep) NEAR/6 (consum* OR eat OR eats OR eating OR ate OR intak* OR purchas* OR 
buy* OR demand* OR choos* OR choice* OR avoid* OR prefer*))) AND noft((emotion* OR affective OR disgust* OR fear OR guilt* OR empath* OR suffer* OR moral* OR ethic* OR humane* OR anthropomorph* OR belief* OR "cognitive dissonan*" OR "meat paradox" OR (animal* NEAR/6 (welfare OR rights OR wellbeing OR "well being" OR cruel* OR abuse OR mistreat*)))

PolicyFile (ProQuest) (((meat* OR "animal product*" OR beef OR veal OR lamb OR pork OR poultry OR chicken OR turkey OR pig OR cow OR sheep) NEAR/6 (consum* OR eat OR eats OR eating OR ate OR intak* OR purchas* OR buy* OR demand* OR choos* OR choice* OR avoid* OR prefer*))) AND noft((emotion* OR affective OR disgust* OR fear OR guilt* OR empath* OR suffer* OR moral* OR ethic* OR humane* OR anthropomorph* OR belief* OR "cognitive dissonan*" OR "meat paradox" OR (animal* NEAR/6 (welfare OR rights OR wellbeing OR "well being" OR cruel* OR abuse OR mistreat*))))

\subsubsection{Methods for searching grey literature}

The grey literature search proceeded in three stages. First, existing resources compiling relevant literature were screened; second, the websites of relevant nonprofits were searched and results screened; and, third, a bibliography of the literature discovered in the first two phases was posted to relevant forums in the animal advocacy research community to solicit more studies. Because titles and abstracts were often unavailable or uninformative in the non-academic grey literature and to simplify communication in the third stage of the search, studies were title and abstract screened to meet only the outcome and design inclusion criteria but with any intervention. All studies passing this broader screening then underwent the same full-text screening by two independent reviewers as used for the peer-reviewed literature.

Existing resources The Humane League Labs' existing database of 781 items was first title and abstract screened and identified studies added to full text screening. This database was generated incidentally through the daily work of three researchers at The Humane League Labs. Researchers added items on a variety of topics as needed during the course of their normal research activities spanning 34 person-months. Next, a variety of other sources known to the author were screened: the background sections from a thorough online report ${ }^{[16]}$, the relevant sections of a popular book ${ }^{96}$, the proceedings of two conferences ${ }^{[23}$, an existing meta-analysis ${ }^{13}$, a data repository ${ }^{60}$, and a website aggregating societally impactful dissertations and theses $\frac{11}{1}$.

Websites of nonprofits A list of animal advocacy and other relevant nonprofits was generated via a snowball sample from author JP's existing database and professional contacts. This process identified 24 organizations for screening, listed in Table S1. The websites of each organization, when available, were first manually searched for relevant sections 
describing research results, and, if such a section were not found, searched for the keywords "study", "research", "survey" and "evaluation". In some cases, individuals at the nonprofit were contacted to solicit additional studies.

Table S1: Websites of nonprofits searched for grey literature

\begin{tabular}{|c|c|}
\hline Organization & URL \\
\hline Albert Schweitzer & https://albertschweitzerfoundation.org/ \\
\hline Anima & https://anima.dk/ \\
\hline Animal Aid & https://www.animalaid.org.uk \\
\hline Animal Charity Evaluators & https://animalcharityevaluators.org/ \\
\hline Animal Equality & https://animalequality.org/ \\
\hline Animal Liberation & https://www.al.org.au/ \\
\hline Animal Welfare Action Lab & http://www.awalab.org/blog/ \\
\hline Animals Australia & https://www.animalsaustralia.org/ \\
\hline Better Buying Lab & $\begin{array}{l}\text { https://www.wri.org/our- } \\
\text { work/project/better-buying-lab/research- } \\
\text { and-resources }\end{array}$ \\
\hline Farm Sanctuary & https://www.farmsanctuary.org/ \\
\hline $\begin{array}{l}\text { Farm Sanctuary Compassionate Communities } \\
\text { Campaign }\end{array}$ & https://ccc.farmsanctuary.org/ \\
\hline Farmed Animal Rights Movement & https://farmusa.org/ \\
\hline Faunalytics & https://faunalytics.org/completed-projects/ \\
\hline Friends of the Earth & https://foe.org/ \\
\hline Humane Society of the United States & https://www.humanesociety.org/ \\
\hline Johns Hopkins Center for a Livable Future & $\begin{array}{l}\text { https://www.jhsph.edu/research/centers- } \\
\text { and-institutes/johns-hopkins-center-for-a- } \\
\text { livable-future/research/clf_publications / }\end{array}$ \\
\hline L214 & https://www.1214.com/ \\
\hline Meatless Monday & https://www.meatlessmonday.com/research/ \\
\hline Mercy for Animals & https://mercyforanimals.org/research \\
\hline One Step for Animals & https://www.onestepforanimals.org/ \\
\hline Pro Veg Netherlands/Viva Las Vega's & None \\
\hline The Humane League Labs & https://thehumaneleague.org/labs \\
\hline Vegan Outreach & https://veganoutreach.org/ \\
\hline Veganuary & https://veganuary.com/ \\
\hline
\end{tabular}

Distribution of bibliography and snowball sampling Finally, a working bibliography of studies identified during the first two stages of the grey literature search was distributed to various forums used in the animal advocacy community on November 20, 2019, which represents the last day of active searching. The bibliography was distributed in an interactive format, using Google Docs, so the community could add missing studies or otherwise comment directly on the document. In particular, the bibliography was distributed to the Farm Animal 
Strategic Team (FAST) email list; PBCM (plant-based clean/cultured/cultivated meat) Consumer Research Collaborative group, hosted on the instant messaging platform Slack; the "Effective Animal Advocacy - Discussion" and "Helping Animals Effectively (Closed)" discussion groups hosted on the social media website Facebook; and several other experts in the field.

\subsection{Details of data extraction}

\subsubsection{Qualitative data extraction}

We extracted the following basic characteristics for each study. Names in monospace represent variable names in the online dataset.

1. unique: A unique identifier (author, year, and substudy if applicable)

2. authoryear: The first author's last name and study year, used as a shorthand identifier

3. substudy: A name or number identifying the study within the article if the article had multiple studies

4. title: The article's title

5. journal and other.source: Journal or non-peer-reviewed source (e.g., technical report)

6. published: An indicator for whether the study was published in a peer-reviewed journal or conference proceedings

7. borderline: Whether the study's eligibility was borderline with respect to inclusion criteria

8. exclude.main: Whether the study was a SSWS study

9. stats.source: How we extracted statistical estimates (from the paper, from publicly available data, or from contacting authors)

10. prose.population: The subject population and recruitment strategy

11. country: The country in which the study was conducted

12. n. paper: The total analyzed number of unique subjects analyzed in the article (rather than the study)

13. perc.male: The percentage of male subjects

14. age: Subjects' mean or median age. When only a range was reported (e.g., "18-35"), we used the midpoint. When the age breakdown was reported by categories (e.g., " $<18$ ", "18-30", ">30"), we estimated the mean age by taking the midpoint of each category (or the minimum of the category in cases such as ">30") and taking a weighted average by the categories' frequencies. When a study recruited only undergraduates and did not report any numerical age statistics, we imputed a mean age of 22 years.

15. students: Subjects' status as college undergraduates. "No": the study did not specifically restrict recruitment to undergraduates (though the sample may have contained undergraduates). "General undergraduates": the study recruited undergraduates generally rather than from a particular major. "Social sciences undergraduates": undergraduates recruited specifically from social sciences majors. When a study recruited undergrad- 
uates but did not indicate that they were specifically social sciences undergraduates, we coded as "general undergraduates". When a study recruited participants from a college or university in a manner that might have included faculty and staff, we coded as "mixed". When a study recruited subjects using an online crowdsourcing platform, did not specifically state that recruitment targeted undergraduates, and had a higher mean age than is typical for undergraduates (e.g., 35 years), we coded as "no".

16. prose.x: A prose description of the intervention

17. prose.control: A prose description of the control group or time period

18. x.tailored: Whether the intervention was personally tailored (i.e., its content varied depending on subjects' individual characteristics, such as their current diets)

19. x.has.text: Whether the intervention contained text

20. x.has.visuals: Whether the intervention contained visuals (e.g., photographs, cartoons, or videos)

21. x.suffer: Whether the intervention contained graphic verbal or visual depictions of factory farm conditions

22. x.pure.animals: Whether the intervention referred only to animal welfare (i.e., it did not make appeals for reduced meat consumption that were not related to animal welfare)

23. x.min.exposed: The estimated total duration of the intervention in minutes, excluding any optional components of the intervention. For interventions involving multiple components, the duration included time during which the subject was actively engaged with components of the intervention and did not include time elapsed between components of the intervention.

24. x.rec: The recommendation made regarding meat consumption ("go vegan", "go vegetarian", "reduce consumption", mixed recommendation, or no recommendation)

25. x.mind.attr: Whether the intervention used mind attribution by describing farm animal's inner states; see Table $\mathrm{S} 2$

26. x.soc.norm: Whether the intervention used social norms (e.g., explicitly describing trends toward decreased meat consumption or describing a group of individuals who do not eat meat); see Table S2

27. x.id.victim: Whether the intervention used the identifiable victim effect by giving a proper-noun name to a farm animal; see Table S2

28. x.impl: Whether the intervention gave implementation suggestions in the form of describing or depciting a specific plant-based meal, restaurant dish, or recipe; see Table S2]

29. x.pets: Whether the intervention described or depicted companion animals that typically live in people's houses, with or without an explicit connection to farm animals; see Table S2

30. y. cat: Whether the outcome related to meat consumption or meat purchase

31. y.lag.days: The number of days elapsed between the end of the intervention and the outcome measurement

32. y.other.eligible.foods: Whether the study measured and reported eligible food outcomes besides the one we extracted based on the rules given in the main text. As described in the main text, these were almost always subscales of a composite that was used as the main outcome. This only includes only other eligible food outcomes, not 
other eligible modes of measuring the outcome (e.g., intentions versus self-reports).

33. prose.y.other.eligible: A prose description of any other eligible food outcomes besides the one we extracted

Because the fine-grained intervention characteristics (i.e., x.mind.attr, x.soc.norm, x.id.victim, $\mathrm{x}$.impl, and $\mathrm{x}$.pets) were more subjective than the other study and intervention characteristics, two authors (DBR and MBM) independently rated them for each study using a standardized set of criteria (Table S2), resolving conflicts through discussion. We assessed interrater reliability using Cohen's $\kappa$, removing "unclear" ratings. $\stackrel{[8]}{\text { The }} \kappa$ estimates were 0.85 for using mind attribution, 0.87 for using social norms, 0.97 for naming an identified victim, 0.95 for giving implementation suggestions, and 0.92 for depicting pets.

We extracted the following characteristics regarding study design, analytic reproducibility, and risks of bias:

1. design: The study design (e.g., randomized controlled trial, cluster-randomized controlled trial, nonrandomized controlled trial)

2. qual.y.prox: The mode of outcome measurement (direct behavioral measure, selfreported behavior, or intended future behavior)

3. qual missing: The percentage of missing data, calculated as the percentage of subjects who received the intervention or control who did not contributed data to our extracted point estimate. For example, for studies in which the intervention involved distributing leaflets on a college campus, the percentage of missing data was coded as the number of subjects providing outcome data divided by the number of leaflets distributed. For studies in which the outcome was measured in the same session as the intervention, we assumed there was no missing data if this information was not reported.

4. qual.prereg: Whether the study's protocol and/or analysis plan were preregistered in any form

5. qual.public.data: Whether the study's data are publicly available

6. qual.public.code: Whether the study's analysis code is publicly available

7. qual. exch: The study's risk of bias with respect to the exchangeability of the intervention and control conditions (low risk, medium risk, high risk, or unclear; see Table S3)

8. qual .gen: The study's risk of bias with respect to external generalizability (low risk, medium risk, high risk, or unclear; see Table S3]

9. qual.sdb: The study's risk of bias with respect to social desirability bias (low risk, medium risk, high risk, or unclear; see Table S3

To develop this list of risk-of-bias criteria, we adapted from the Cochrane Risk of Bias Tool for randomized studies ${ }^{[15}$ those criteria that were relevant to the present literature (e.g., criteria related to selection bias, attrition bias, and reporting bias), removed some criteria that were not relevant or feasible for this literature (e.g., regarding blinding), and expanded the generic category "Other bias" into criteria of particular relevance to this literature (e.g., regarding the quality of outcome measurement and the potential for social desirability bias). We adapted the existing items in order, for example, to allow assessment of the stated type of bias in the 
diverse study designs that we expected to encounter. To develop the tool, a group of authors (MBM, JP, DBR, JN) read studies known to meet eligibility criteria and, based on their combined expertise in meta-analysis methodology and in research on interventions to reduce meat consumption, developed the risk of bias tool through discussion, piloting on eligible studies, and iterative revision. To extract these qualitative data, one author (MBM or DBR) extracted data for each study, emailing study authors as needed to fill in missing information. The other author confirmed the entries. For three risk-of-bias criteria that were somewhat subjective and difficult to assess (exchangeability, avoidance of social desirability bias, and external generalizability), two authors (among DBR, JN, and MBM) independently rated the article using a standardized set of criteria (Table S3), resolving conflicts through discussion or adjudication by another author. We assessed interrater reliability using a weighted Cohen's $\kappa$ to accommodate the ordinal nature of the ratings, again removing "unclear" ratings. ${ }^{8}$ Because the three raters rated only partly overlapping subsets of studies, we calculated $\kappa$ for each of the three pairs of raters. The mean $\kappa$ was 0.27 for social desirability bias, 0.18 for external generalizability, and 0.21 for exchangeability.

We extracted the following statistical information:

1. effect.measure: The scale of the initial point estimate yi and variance vi (standardized mean difference or risk ratio) prior to any needed conversion to the risk ratio scale

2. desired.direction: Whether the point estimate was in the desired direction (i.e., reducing rather than increasing meat consumption or purchase)

3. yi: The point estimate on the scale described above after synchronizing estimate directions using desired.direction

4. vi: The variance estimate on the scale described above

5. interpretation: A prose interpretation of the extracted estimate prior to conversion to the log-risk ratio scale

6. logRR: The point estimate converted (if needed) to the log-risk ratio scale; this was the estimate used in meta-analysis

7. varlogRR: The variance estimate converted (if needed) to the log-risk ratio scale; this was the estimate used in meta-analysis

8. RR. 10: The lower limit of a $95 \%$ confidence interval for the estimate on the risk ratio for use in the forest plot

9. RR.hi: The upper limit of a $95 \%$ confidence interval for the estimate on the risk ratio for use in the forest plot 


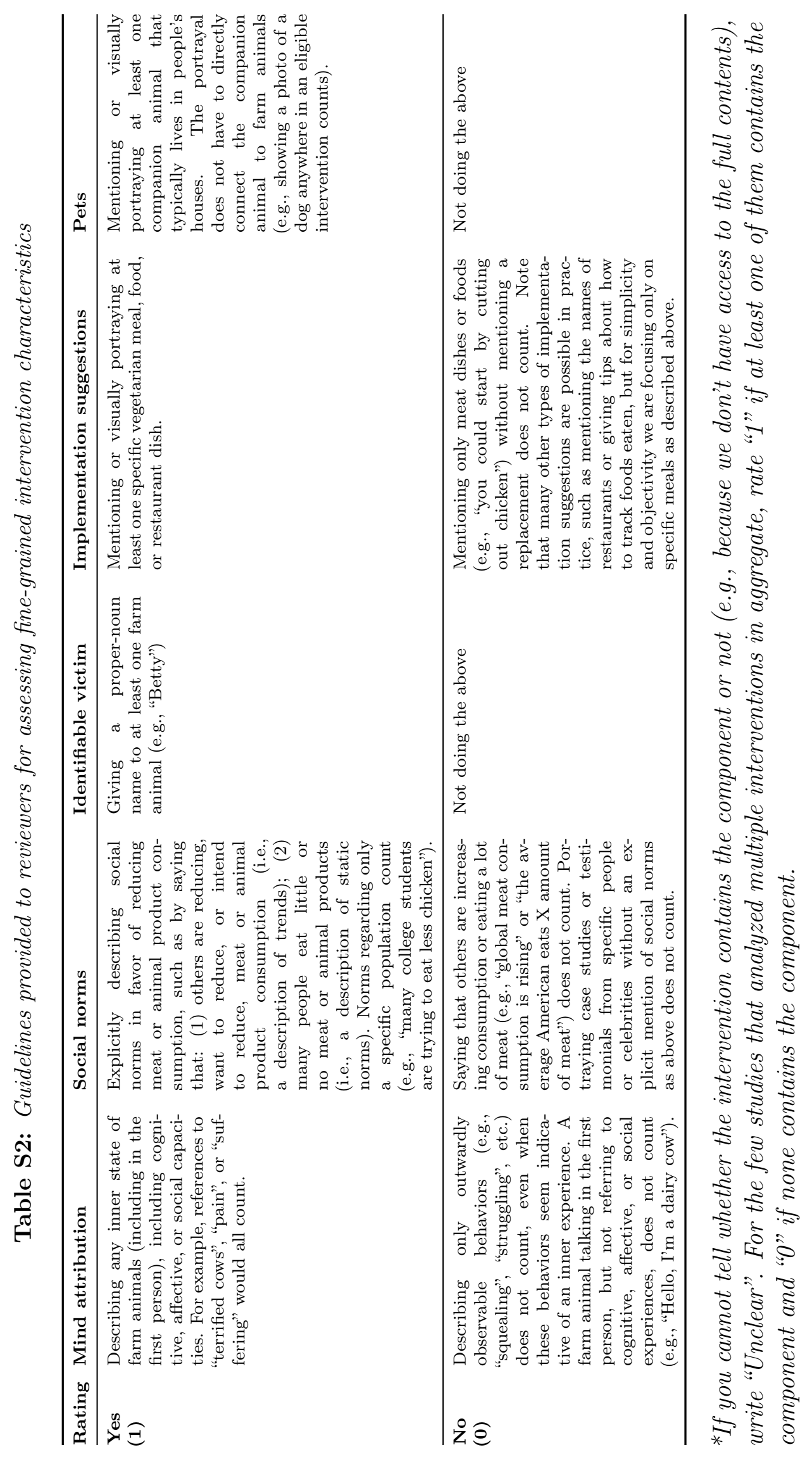




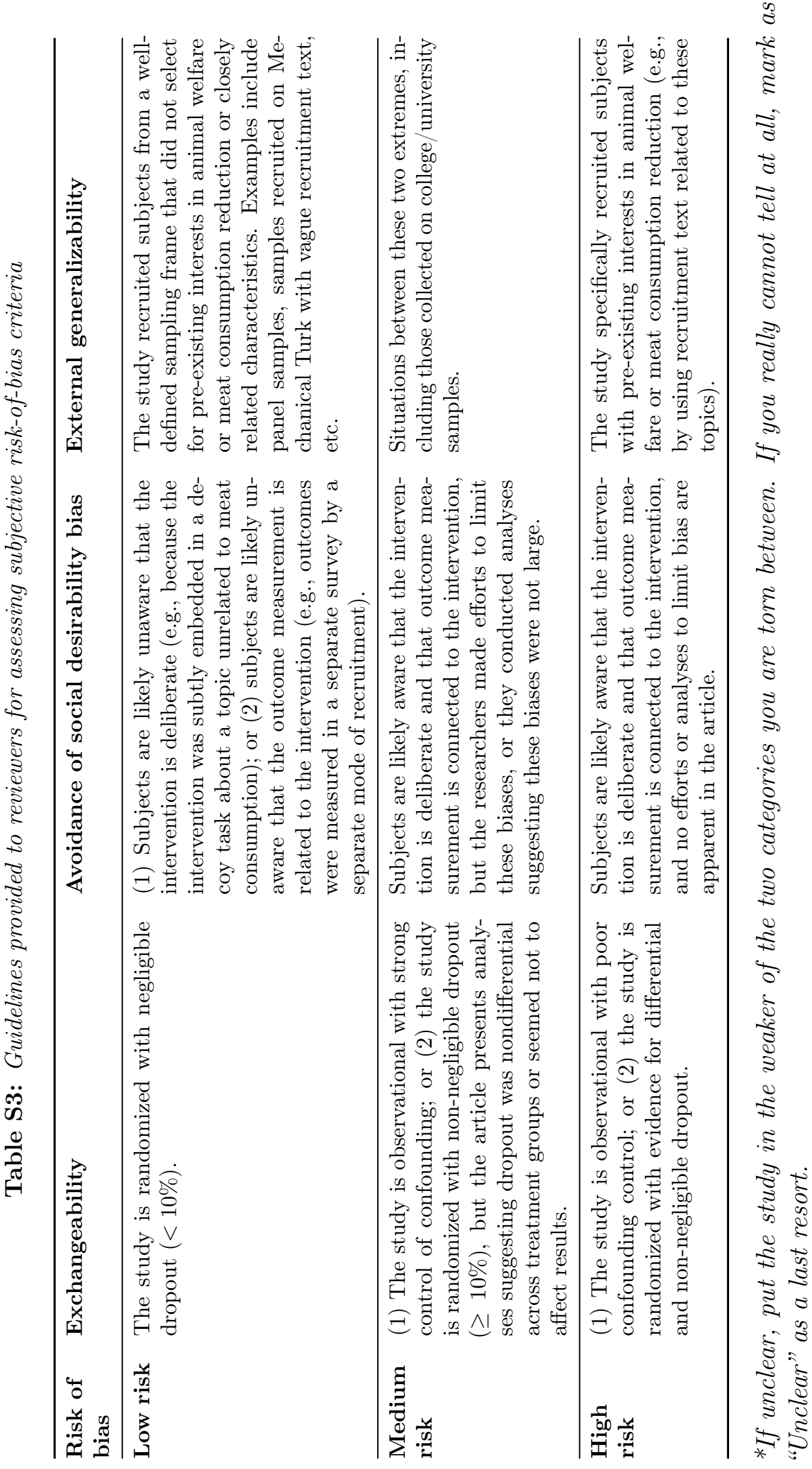




\subsubsection{Quantitative data extraction}

To ensure that point estimates were statistically and conceptually comparable across studies, we extracted, when feasible, point estimates for each study that represented the risk ratio of "low" versus "high" meat consumption or purchase as follows. For studies reporting sufficient information or with raw data available, we defined "low" and "high" relative to the median consumption or purchasing outcome in the control condition or time period (e.g., "high" consumption for a two-group randomized trial would be defined as consumption higher than the median consumption in the control group). For studies with outcomes measured before the intervention, we used the baseline median in all subjects regardless of intervention group. Dichotomizing at the median, rather than at a more extreme value, improves statistical precision.

When raw data were available, we estimated risk ratios as follows. For studies with a single wave of data collection after the intervention, we simply estimated a marginal risk ratio for low vs. high consumption. For studies with baseline outcome measures available, we controlled for these measures by estimated the conditional risk ratio via Poisson, using ${ }^{[27}$ s $\mathrm{S}$ heteroskedasticity-consistent standard error estimator ${ }^{[20}$. Controlling for baseline outcome measures increases statistical precision and helps control for confounding. For studies that used a within-subjects design without a separate control group, we calculated matched-pairs risk ratios per ${ }^{[28]}$ using the $\mathrm{R}$ package metafor ${ }^{[26]}$.

Directly estimating the risk ratio for low versus high consumption as defined above was sometimes not feasible when data were not available. In this case, if the study reported a binary outcome corresponding conceptually to "low" versus "high" meat consumption (e.g., being vegetarian vs. not being vegetarian; or reducing meat consumption from one's previous consumption versus maintaining or increasing consumption), we extracted the corresponding risk ratio. If instead the study reported a continuous outcome (e.g., servings of meat consumed), we calculated a point estimate on the standardized mean difference scale and then approximately converted the estimate to the risk ratio scale as follows. Letting $d$ denote a standardized mean difference, the approximate odds ratio arising from dichotomizing the (approximately logistically distributed) outcome at an arbitrary cut point is $\log O R \approx \pi d / \sqrt{3^{144}}$. If we assume the cut point is chosen to be near the median of the continuous outcome, in keeping with the convention we used when calculating risk ratios directly, then the odds ratio is anticonservative for the risk ratio but can be approximately converted via the transformation $\log R R \approx \log \sqrt{\exp \{\log O R\}^{[22}}$. We therefore have $\log R R \approx \log (\sqrt{\exp \{\pi d / \sqrt{3}\}})$ and, by the delta method, $\operatorname{Var}(\log R R) \approx\left(\pi^{2} / 12\right) \operatorname{Var}(d)$.

For one study that reported a point-biserial correlation ${ }^{[12}$, we approximated the variance as $\operatorname{Var}\left(r_{p b}\right) \approx\left(1-r_{p b}^{2}\right)^{2} /\left(n_{1}+n_{0}-1\right)$, where $r_{p b}$ is the point-biserial correlation and $n_{0}$ and $n_{1}$ are the sample sizes in each exposure group ${ }^{[17}$. We converted to the standardized mean 
difference scale per reference ${ }^{17}$ :

$$
d \approx \frac{r_{p b} \sqrt{h}}{\sqrt{1-r_{p b}^{2}}}
$$

where $h=\frac{n_{1}+n_{0}-2}{n_{1}}+\frac{n_{1}+n_{0}-2}{n_{0}}$. We again used the delta method to convert the variance. We then converted these measures on the standardized mean difference to the log-risk ratio scale as above.

\subsection{Details of sensitivity analysis for social desirability bias}

To conduct the sensitivity analyses for social desirability bias across studies, we first used a nonparametric method that conducts a grid search across hypothetical values of bias severity across studies, in which bias severity is operationalized as the multiplicative factor by which bias has inflated each study's point estimate away from the null. ${ }^{19}$ We conducted a separate grid search for each of the two sensitivity analyses (regarding bias required to shift the estimate to the null and to reduce to only $10 \%$ the percentage of true population effects stronger than $R R=1.1$ ); we obtained confidence intervals using bias-corrected and accelerated bootstrapping. ${ }^{19}$ Those methods in fact apply for any type of internal bias that affects estimates multiplicatively and whose severity is independent of the studies' causal (i.e., unbiased) population effects. The multiplicative bias that can be produced by differential measurement of the outcome is, at maximum, equal to the strength of the direct effect of the exposure on the mismeasured outcome, not through the correctly measured outcome. ${ }^{23}$ Given the conception of social desirability bias given in the main text, it follows immediately that the severity of social desirability bias required to produce any given multiplicative bias factor is simply equal to the bias factor itself.

\section{SUPPLEMENTARY RESUlts}

\subsection{Reference lists of included articles}

\subsubsection{Articles included in main analysis}

Amiot, C. E., Boutros, G. E. H., Sukhanova, K., \& Karelis, A. D. (2018). Testing a novel multicomponent intervention to reduce meat consumption in young men. PloS One, 13 (10), e0204590.

Anderson, E. C., \& Barrett, L. F. (2016). Affective beliefs influence the experience of eating meat. PLoS One, 11 (8), e0160424. 
Anderson, J. (2017). An experimental investigation of the impact of video media on pork consumption (Tech. Rep.). Faunalytics. (https://drive.google.com/file/d/1ovZ0xvJPK LGayAX-uCyGuZtJKCFcYOTP/view)

Animal Charity Evaluators. (2013a). 2013 Humane Education Study (Tech. Rep.). (2013). (https://animalcharityevaluators.org/advocacy-interventions/interventions/h umaneeducation/2013-humane-education-study/)

Animal Charity Evaluators. (2013b). 2013 Leafleting Study (Tech. Rep.). (2013). (https: //animalcharityevaluators .org/advocacyinterventions/interventions/leafleting /2014-march/2013-leafleting-study/)

Bertolaso, C. (2015). Investigating the effectiveness of message framing and regulatory fit in increasing positive animal attitude and reducing animal products consumption: A study for animal equality (Unpublished master's thesis). Universiteit van Amsterdam. (https: //scripties.uba.uva.nl/scriptie/570273)

Byrd-Bredbenner, C., Grenci, A.,\& Quick, V. (2010). Effect of a television programme on nutrition cognitions and intended behaviours. Nutrition and Dietetics, 67 (3), 143-149.

Caldwell, K. (2016, November). Welfare reforms and meat consumption (Tech. Rep.). Mercy for Animals. (https://mercyforanimals.org/welfare-reforms-survey)

Caldwell, K. (2017, March). Which kinds of pro-vegetarian videos are best at inspiring changes in diets and attitudes? (Tech. Rep.). Mercy for Animals. (https://mercyforanimals.org/ kinds-of-viral-videos-are-best-at-inspiring)

Cooney, N. (2014, March). What elements make a vegetarian leaflet more effective? (Tech. Rep.). Humane League Labs. (http://www .humaneleaguelabs.org/blog/2014-05-20-w hatelements-make-vegetarian-leaflet-more-effective/)

Cooney, N. (2016, March). Do online videos of farmed animal cruelty change people's diets and attitudes? (Tech. Rep.). Mercy for Animals. (https://mercyforanimals.org/impact -study)

Cordts, A., Nitzko, S.,\& Spiller, A. (2014). Consumer response to negative information on meat consumption in Germany. International Food and Agribusiness Management Review, 17 (Special Issue A), 83-106.

da Silva, C. R. (2016). Am I too cute to eat? The effect of cuteness appeal towards the promotion of a more plant-based diet (Unpublished master's thesis). University Institute of Lisbon. (https://repositorio.iscteiul.pt/bitstream/10071/12583/1/Vers\%C3\%A30\% 20completa\%20disserta\%C3\%A7\%C3\%A30\%20MPSOCatarina\%20Silva.pdf)

Doebel, S., Gabriel, S.,\& The Humane League. (2015, August). Does encouraging the public to "Eat vegan", "Eat vegetarian", "Eat less meat", or "Cut out or cut back on" meat and other animal products lead to the most diet change? (Tech. Rep.). Humane League Labs. 
(http://www.humaneleaguelabs.org/static/reports/E006R01-which-request-create sthe-most-diet-change.pdf)

Earle, M., Hodson, G., Dhont, K.,\& MacInnis, C. (2019). Eating with our eyes (closed): Effects of visually associating animals with meat on antivegan/vegetarian attitudes and meat consumption willingness. Group Processes and Intergroup Relations, 22(6), 1-18.

Feltz, A., Feltz, S., Cogley, Z., Caton, J., Engel, M., Ilea, R., Johnson, L. S. (2019, October). Animal product consumption: measurement and education (Workshop). In Eating meat 2019 - determinants, consequences and interventions. DIW Berlin, 8-9 October 2019.

FIAPO. (2017) Effectiveness assessment of outreach methods used by Federation of Indian Animal Protection Organisations (FIAPO) (Tech. Rep.). (2017). (New Delhi, India)

Flens, G., Moleman, P.,\& de Rooy, L. (2018, March). The effectiveness of leafletting on reducing the consumption of animal products in Dutch students (Tech. Rep.). Stichting Viva las Vega's and Animal Rights Netherlands Belgium. (https://osf.io/6tfa9/)

Hennessy, S. R. (2016). The impact of information on animal product consumption (Unpublished master's thesis). University of Illinois at Urbana Champaign. (https://www.ideals . illinois.edu/bitstream/handle/2142/92865/HENNESSYTHESIS-2016.pdf? sequence= 1isAllowed=y)

Kunst, J. R.,\& Haugestad, C. A. P. (2018). The effects of dissociation on willingness to eat meat are moderated by exposure to unprocessed meat: A cross-cultural demonstration. Appetite, emph120, 356-366.

Kunst, J. R.,\& Hohle, S. M. (2016). Meat eaters by dissociation: How we present, prepare and talk about meat increases willingness to eat meat by reducing empathy and disgust. Appetite, 105, 758-774.

Lackner, Z. (2019). The effect of dissociation between meat and animal on the experience of ambivalence towards meat. (Unpublished manuscript) (Université de Grenoble Alpes)

Macdonald, B., Caldwell, K.,\& Boese, G. (2016). The effects of 'reduce' and 'eliminate' appeals on individual meat consumption (Tech. Rep.). Reducetarian Foundation. (https: //osf.io/nxrx3/)

Norris, J. (2014). Booklet comparison study: Pay Per Read \#1 (Tech. Rep.). Vegan Outreach. (https://veganoutreach.org/pay-per-read-study-results/)

Norris, J.,\& Hannan, R. (2019). Leafleting and booklet effectiveness (Tech. Rep.). Vegan Outreach. (https://veganoutreach.org/leafleting-and-booklet-effectiveness/)

Norris, J.,\& Roberts, E. (2016, July). Booklet comparison study - Pay Per Read \#2 (Tech. Rep.). Vegan Outreach. 
Novatná, A. (2019). The influence of movie on behavioral change in individual meat and dairy products consumption (Unpublidshed Bachelor's Thesis). Masaryk University, Brno, Czech Republic. (https://is.muni.cz/th/cfyr2/Novotnabakalarskaprace.pdf)

Palomo-Vélez, G., Tybur, J. M.,\& van Vugt, M. (2018). Unsustainable, unhealthy, or disgusting? Comparing different persuasive messages against meat consumption. Journal of Environmental Psychology, 58, 63-71.

Reese, J. (2015, March). Testing the effectiveness of animal advocacy messages with Amazon Mechanical Turk (Tech. Rep.). The Sentience Institute. (https://jacyreese.com/assets /downloads/Testing_the_Effectiveness.pdf)

Rouk, S. (2017, August). Which farmed fish welfare issues does the public care most about? (Neutral messaging) (Tech. Rep.). Mercy for Animals. (https://mercyforanimals.org/wh ichfish-welfare-issue-generates-the-most)

Schnabelrauch Arndt, C. A. (2016). Tailoring feedback and messages to encourage meat consumption reduction (Unpublished doctoral dissertation). Kansas State University. (https: //krex.k-state.edu/dspace/handle/2097/32159)

Schwitzgebel, E., Cokelet, B.,\& Singer, P. (2019, July). Ethics classes can influence student behavior: Students purchase less meat after discussing arguments for vegetarianism. The Splintered Mind. (http://schwitzsplinters.blogspot.com/2019/07/ethics-classes-c aninfluence-student.html)

Spanikova, S. (2015). Reducing meat consumption: An information-based intervention. (Unpublished undergraduate honors dissertation) University of Edinburgh. (https://era ed.ac.uk/handle/1842/16745?show=full).

Tian, Q., Hilton, D.,\& Becker, M. (2016). Confronting the meat paradox in different cultural contexts: Reactions among Chinese and French subjects. Appetite, 96, 187-194.

\subsubsection{Articles reporting on SSWS studies}

Faunalytics. (2015). Veganuary (Tech. Rep.). Veganuary in association with Viva! (https: //faunalytics.org/wp-content/uploads/2015/05/Citation2562Veganuary2014Infogr aphic.pdf)

Faunalytics. (2016, March). Veganuary 2016: 6-month survey results (Tech. Rep.). Veganuary. (https://animalcharityevaluators .org/wp-content/uploads/2016/11/Veganua ry-results-six-month-follow-up-2016.pdf)

Faunalytics. (2019, June). Challenge 22+ pilot impact study (Tech. Rep.). Faunalytics. (https://faunalytics.org/challenge-22-pilot-impact-study/) 
Grassian, T.(2019, March). Meat reduction vegan promotion (Tech.Rep.). For Us All (Blog). (https://forusallsite.files.wordpress.com/2019/03/grassian-reductionandvega npromotion-v2.pdf)

Mensink, R. (2017). Becoming vegan. factors influencing reduction of meat and dairy consumption by participants of the VeggieChallenge (Bachelor thesis). Utrecht University,Netherlands. (https://osf.io/b3xzs)

Moleman, P. (2018, March). Impact evaluation of 30-day VeggieChallenge, 2017 (Tech. Rep.). Viva las Vega's (Proveg). (https://osf.io/q62uk)

Vegan Outreach. (2019, July). Impact of 10 Weeks to Vegan (Tech. Rep.). (https: //veganoutreach.org/10wimpact/)

\subsection{Supplementary tables}

Table S4: Articles from which we could not extract statistics for inclusion in the meta-analysis. ID: First author's last name for published studies or name of challenge (organization) for unpublished studies. SSWS: indicator for whether the study was a self-selected withinsubjects study and hence would have been omitted from main analyses even if statistics had been available.

\begin{tabular}{|c|c|c|c|}
\hline$\overline{\mathbf{I D}}$ & Year & SSWS & Results reported \\
\hline $\operatorname{de~Lanauze}^{10}$ & 2019 & No & $\begin{array}{l}\text { In the intervention group, motivations to reduce } \\
\text { meat consumption increased from } 4.08 \text { on a } 7 \\
\text { point Likert scale before the intervention to } 4.15 \\
\text { after the intervention }(p=0.70) \text {. In the control } \\
\text { group, motivations decreased from } 4.12 \text { to } 3.81 \\
(p=0.37) \text {. }\end{array}$ \\
\hline$\overline{\text { Dowsett }}^{[11]}$ & 2018 & No & $\begin{array}{l}\text { None relevant. The eligible outcome measure was } \\
\text { a free-text response to "Will you continue eating } \\
\text { meat with the same regularity?" that was not } \\
\text { coded quantitatively. }\end{array}$ \\
\hline $\begin{array}{l}\text { Summer Vegan Pledge } \\
{\text { (Animal Aid) })^{4}}^{\text {Animal }}\end{array}$ & 2019 & Yes & $\begin{array}{l}57 \% \text { of subjects intended to stay vegan after the } \\
\text { challenge. } 35 \% \text { of subjects reported reducing their } \\
\text { animal product consumption. }\end{array}$ \\
\hline 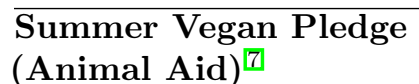 & 2018 & Yes & $\begin{array}{l}53 \% \text { of subjects reported staying vegan after } 6 \\
\text { months. }\end{array}$ \\
\hline $\begin{array}{l}\text { Veganuary } \\
\text { (Veganuary) }^{25}\end{array}$ & 2019 & Yes & $\begin{array}{l}47 \% \text { of subjects intended to stay vegan after the } \\
\text { challenge. }\end{array}$ \\
\hline $\begin{array}{l}\text { Veganuary } \\
(\text { Veganuary })^{21}\end{array}$ & 2018 & Yes & $\begin{array}{l}62 \% \text { of subjects intended to stay vegan after the } \\
\text { challenge. }\end{array}$ \\
\hline $\begin{array}{l}\text { Veganuary } \\
(\text { Veganuary })^{24}\end{array}$ & 2017 & Yes & $\begin{array}{l}67 \% \text { of subjects intended to stay vegan after the } \\
\text { challenge. }\end{array}$ \\
\hline
\end{tabular}


Table S5: Pairwise Pearson's correlations ( $r$ ) between meta-regressive covariates among studies with pairwise complete data, displayed in descending order of correlation. The categorical variable regarding the type of recommendation made is dichotomized here as "making any request" versus "making no request" for brevity.

\begin{tabular}{|c|c|c|}
\hline Variable 1 & Variable 2 & $r$ \\
\hline Social norms & Implementation suggestions & 0.82 \\
\hline Pets & Implementation suggestions & 0.82 \\
\hline Implementation suggestions & Follow-up $\geq 7$ days & 0.81 \\
\hline Pets & Follow-up $\geq 7$ days & 0.75 \\
\hline Social norms & Follow-up $\geq 7$ days & 0.74 \\
\hline Intervention $>5$ min & Follow-up $\geq 7$ days & 0.66 \\
\hline Social norms & Pets & 0.64 \\
\hline Social norms & Makes request & 0.61 \\
\hline Implementation suggestions & Makes request & 0.57 \\
\hline Visuals & Follow-up $\geq 7$ days & 0.56 \\
\hline Visuals & Implementation suggestions & 0.55 \\
\hline Pets & Makes request & 0.54 \\
\hline Makes request & Follow-up $\geq 7$ days & 0.54 \\
\hline Visuals & Pets & 0.53 \\
\hline Pets & Intervention $>5$ min & 0.53 \\
\hline ID victim & Pets & 0.53 \\
\hline Mind attribution & Follow-up $\geq 7$ days & 0.52 \\
\hline Graphic & Mind attribution & 0.52 \\
\hline Text & Mind attribution & 0.52 \\
\hline Graphic & Social norms & 0.51 \\
\hline ID victim & Intervention $>5$ min & 0.50 \\
\hline Implementation suggestions & Intervention $>5$ min & 0.47 \\
\hline Mind attribution & Makes request & 0.47 \\
\hline Visuals & Social norms & 0.46 \\
\hline Mind attribution & Social norms & 0.46 \\
\hline Mind attribution & Pets & 0.43 \\
\hline Visuals & Intervention $>5 \mathrm{~min}$ & 0.42 \\
\hline Graphic & Implementation suggestions & 0.42 \\
\hline Mind attribution & Implementation suggestions & 0.41 \\
\hline Social norms & Intervention $>5$ min & 0.40 \\
\hline Text & Graphic & 0.40 \\
\hline Text & Makes request & 0.40 \\
\hline Male & Age & 0.40 \\
\hline ID victim & Follow-up $\geq 7$ days & 0.37 \\
\hline Mind attribution & ID victim & 0.36 \\
\hline ID victim & Implementation suggestions & 0.36 \\
\hline Social norms & ID victim & 0.33 \\
\hline Graphic & Follow-up $\geq 7$ days & 0.33 \\
\hline Graphic & ID victim & 0.28 \\
\hline
\end{tabular}




\begin{tabular}{ccc} 
Text & Social norms & 0.27 \\
Graphic & Pets & 0.26 \\
Graphic & Male & 0.25 \\
Makes request & Intervention $>5$ min & 0.23 \\
Mind attribution & Intervention $>5$ min & 0.22 \\
Text & Implementation suggestions & 0.20 \\
Graphic & Makes request & 0.19 \\
Visuals & ID victim & 0.18 \\
Mind attribution & Male & 0.17 \\
ID victim & Makes request & 0.17 \\
Visuals & Makes request & 0.17 \\
Text & Pets & 0.15 \\
Text & ID victim & 0.14 \\
Text & Follow-up $\geq 7$ days & 0.13 \\
Graphic & Intervention $>5$ min & 0.13 \\
Text & Male & 0.11 \\
Visuals & Mind attribution & 0.10 \\
Text & Age & 0.07 \\
Text & Intervention $>5$ min & 0.06 \\
Social norms & Male & 0.03 \\
Visuals & Graphic & 0.02 \\
ID victim & Age & -0.03 \\
Implementation suggestions & Male & -0.03 \\
ID victim & Male & -0.05 \\
Graphic & Age & -0.10 \\
Makes request & Male & -0.13 \\
Pets & Male & -0.19 \\
Mind attribution & Age & -0.25 \\
Makes request & Age & -0.26 \\
Text & Visuals & -0.32 \\
Follow-up $\geq 7$ days & Male & -0.34 \\
Visuals & Male & -0.36 \\
Social norms & Age & -0.38 \\
Intervention $>5$ min & Age & -0.46 \\
Intervention $>5$ min & Male & -0.48 \\
Visuals & Age & -0.51 \\
Pets & Age & -0.56 \\
Follow-up $\geq 7$ days & Age & -0.61 \\
Implementation suggestions & Age & -0.65 \\
\hline & & \\
Tale & &
\end{tabular}




\subsection{Supplementary figures}

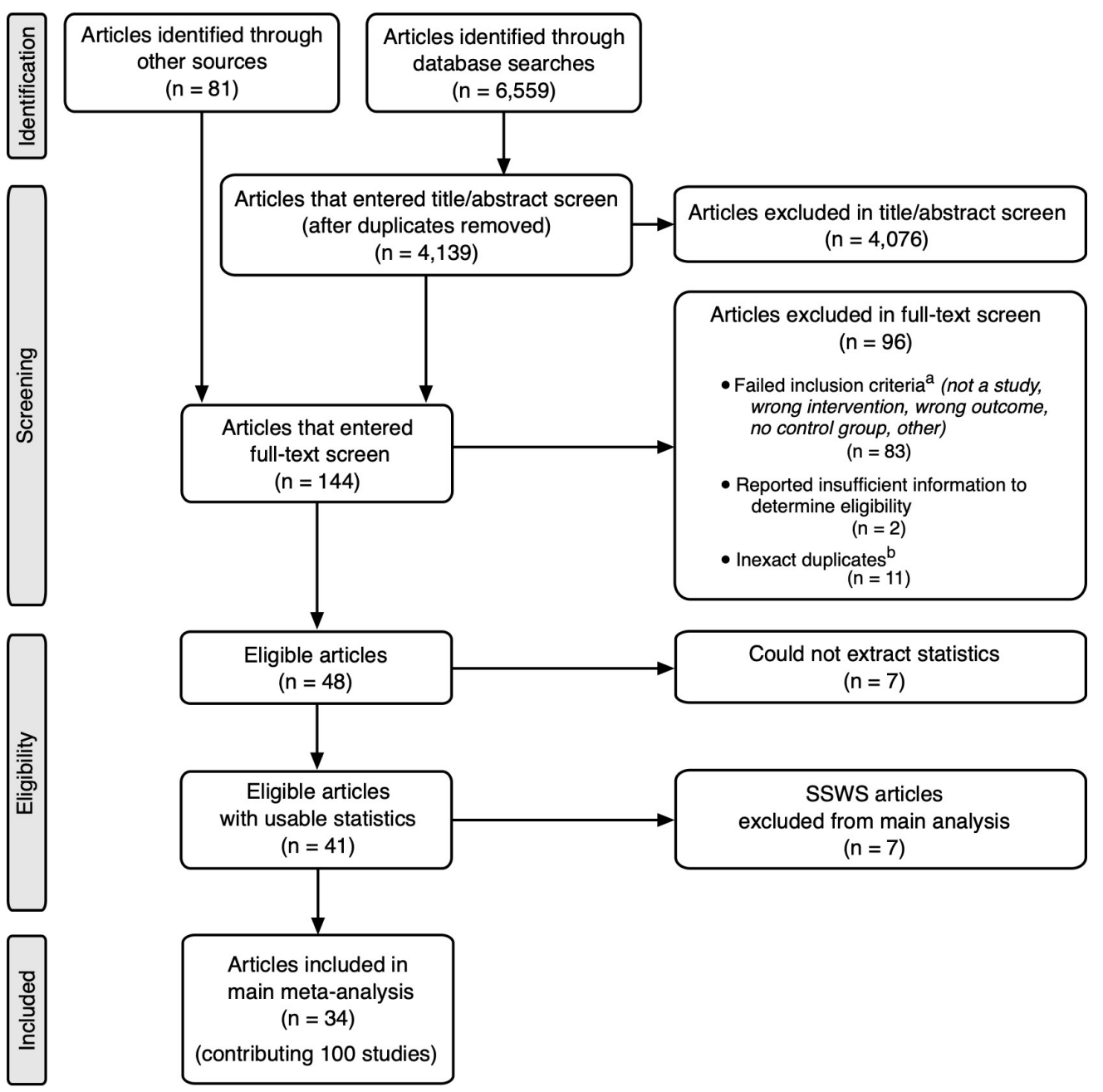

Figure S1: PRISMA flowchart depicting article screening, inclusion, and exclusion decisions. ${ }^{a}$ : Listed reasons are shorthand for the criteria in the main text, which reviewers could view when screening articles. SSWS: self-selected within-subjects studies, as defined in the main text. ${ }^{b}$ : Inexact duplicates are distinct articles referring to the same data, such as an initial report and a re-analysis of the same data. Identifying such articles was possible only upon full-text screening and comparison across screened articles. 


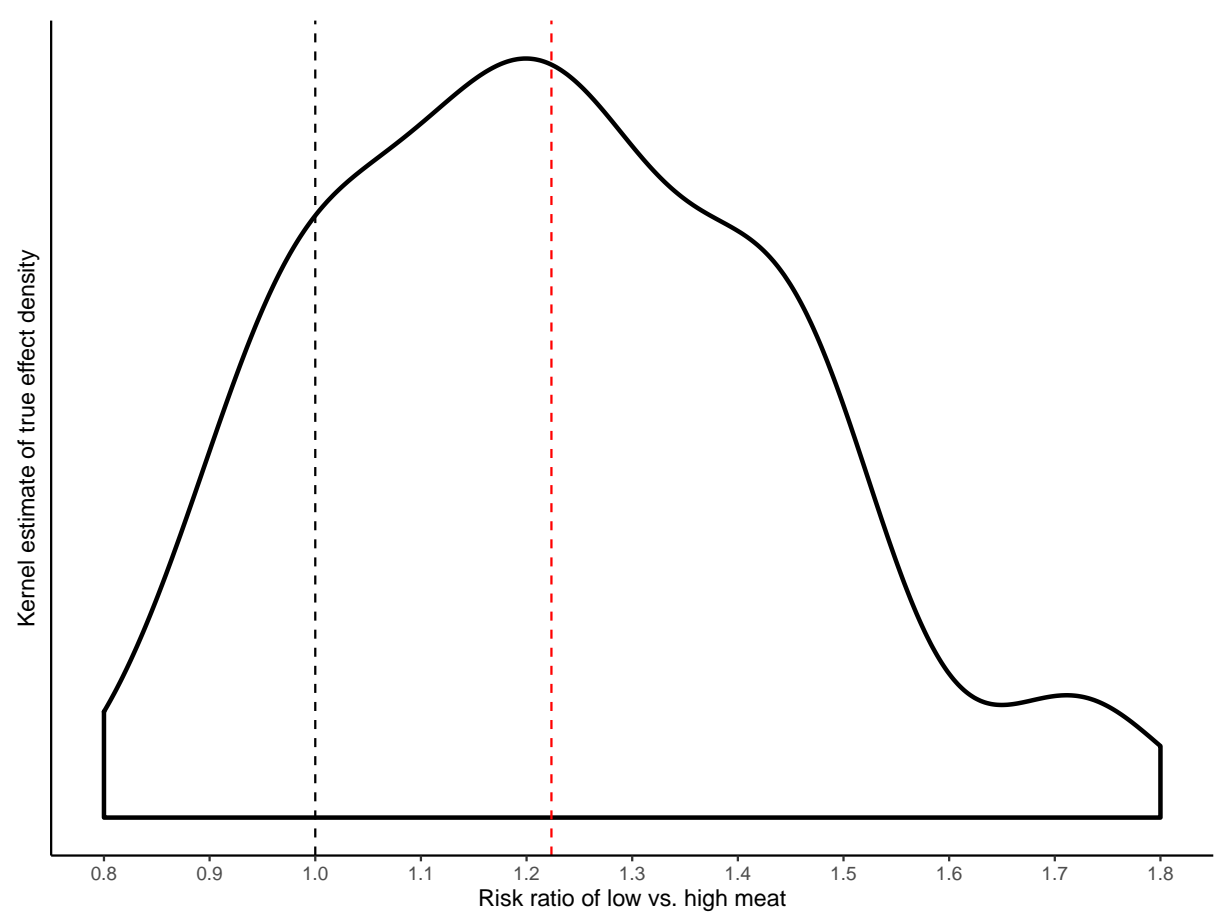

Figure S2: Kernel density estimate of the true risk ratios in each study, truncated to show risk ratios between 0.8 and 1.8. The black dashed line represents the null (no intervention effect). The red dashed line represents the pooled point estimate. 


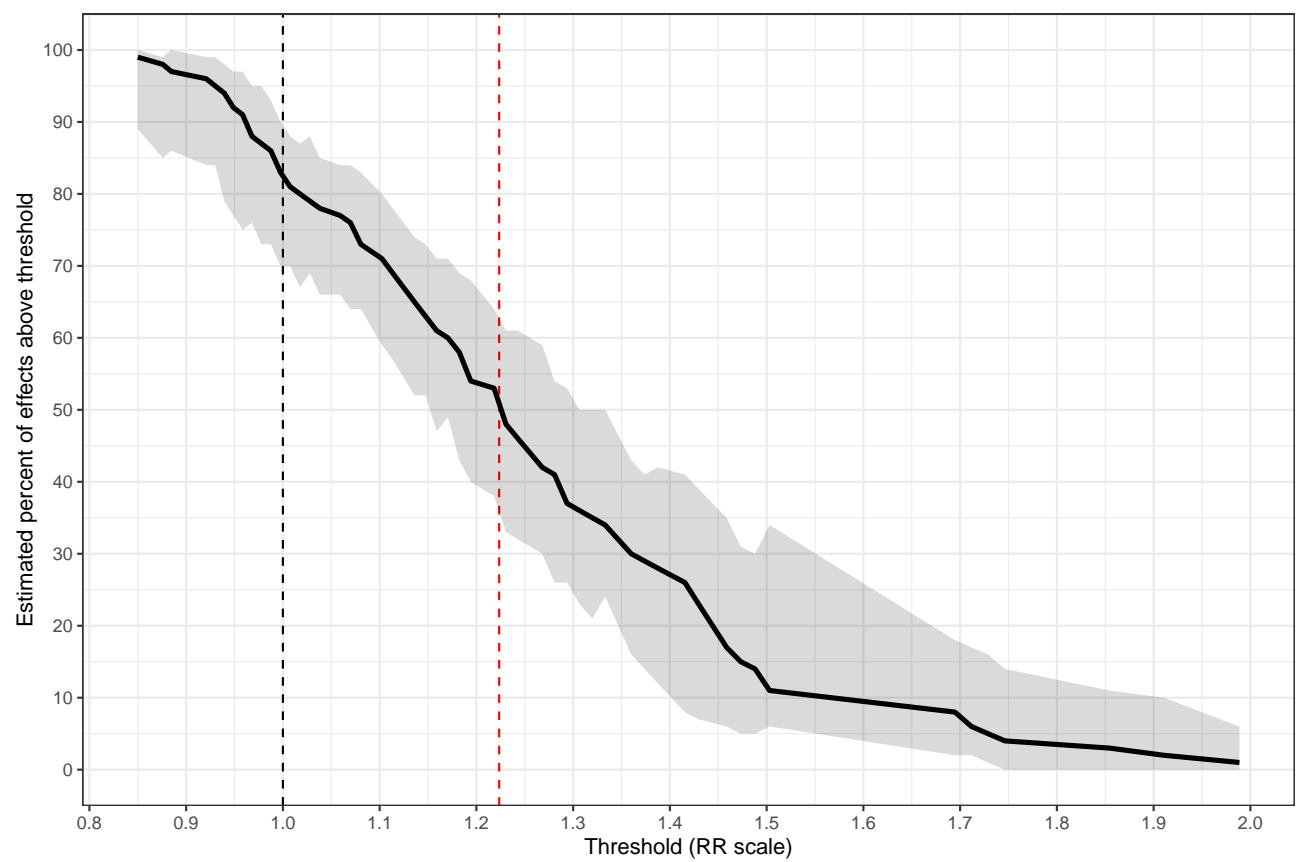

Figure S3: Estimated percentages of true population effects stronger than various thresholds on the risk ratio scale. The black dashed line represents the null (no intervention effect). The red dashed line represents the pooled point estimate. Shaded bands are $95 \%$ bootstrapped pointwise confidence intervals.
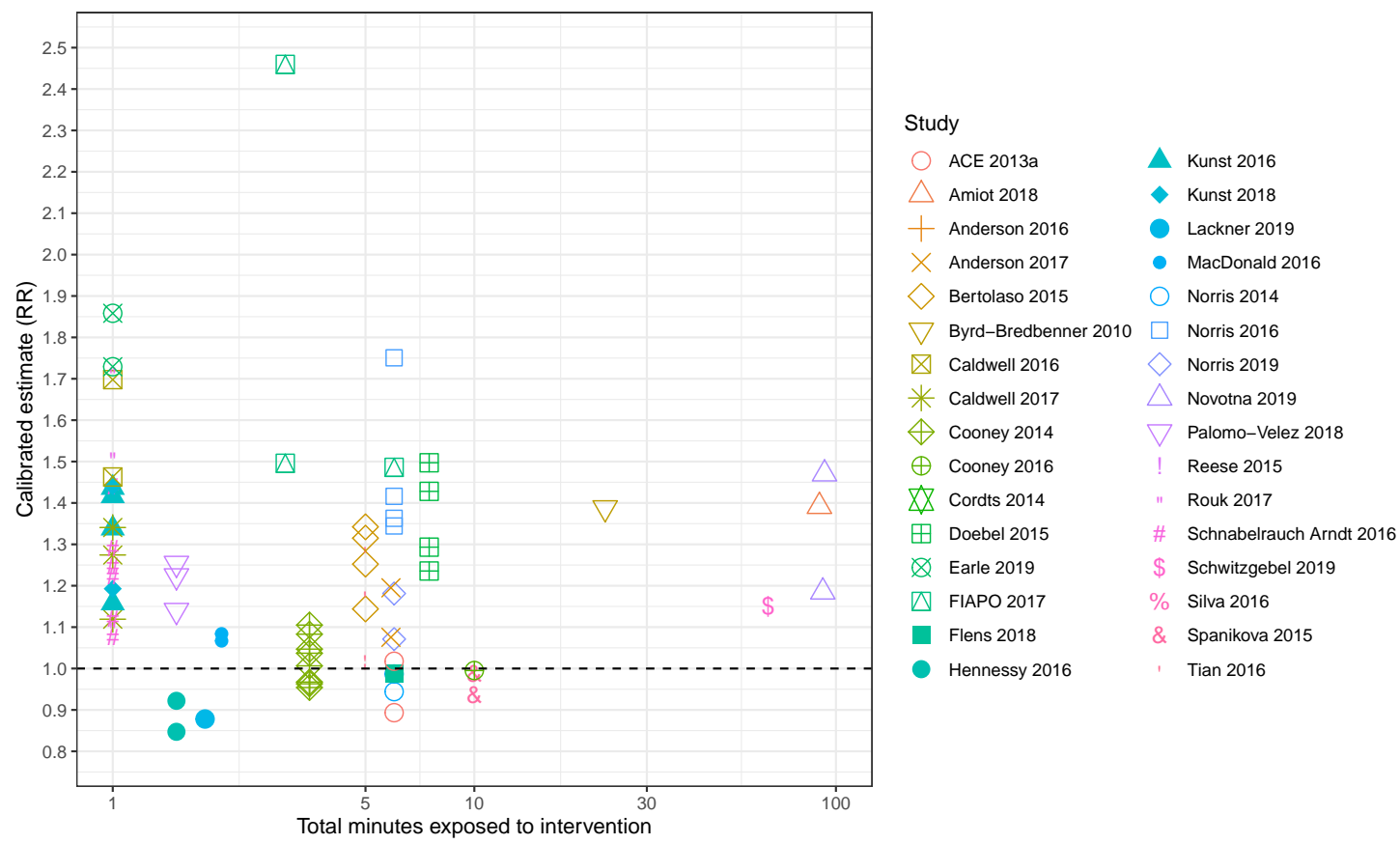

Figure S4: Intervention duration versus calibrated estimate of true population effect size. Horizontal dashed line: null. The $x$-axis is presented on the log scale with numerical marks on the original (minutes) scale. 


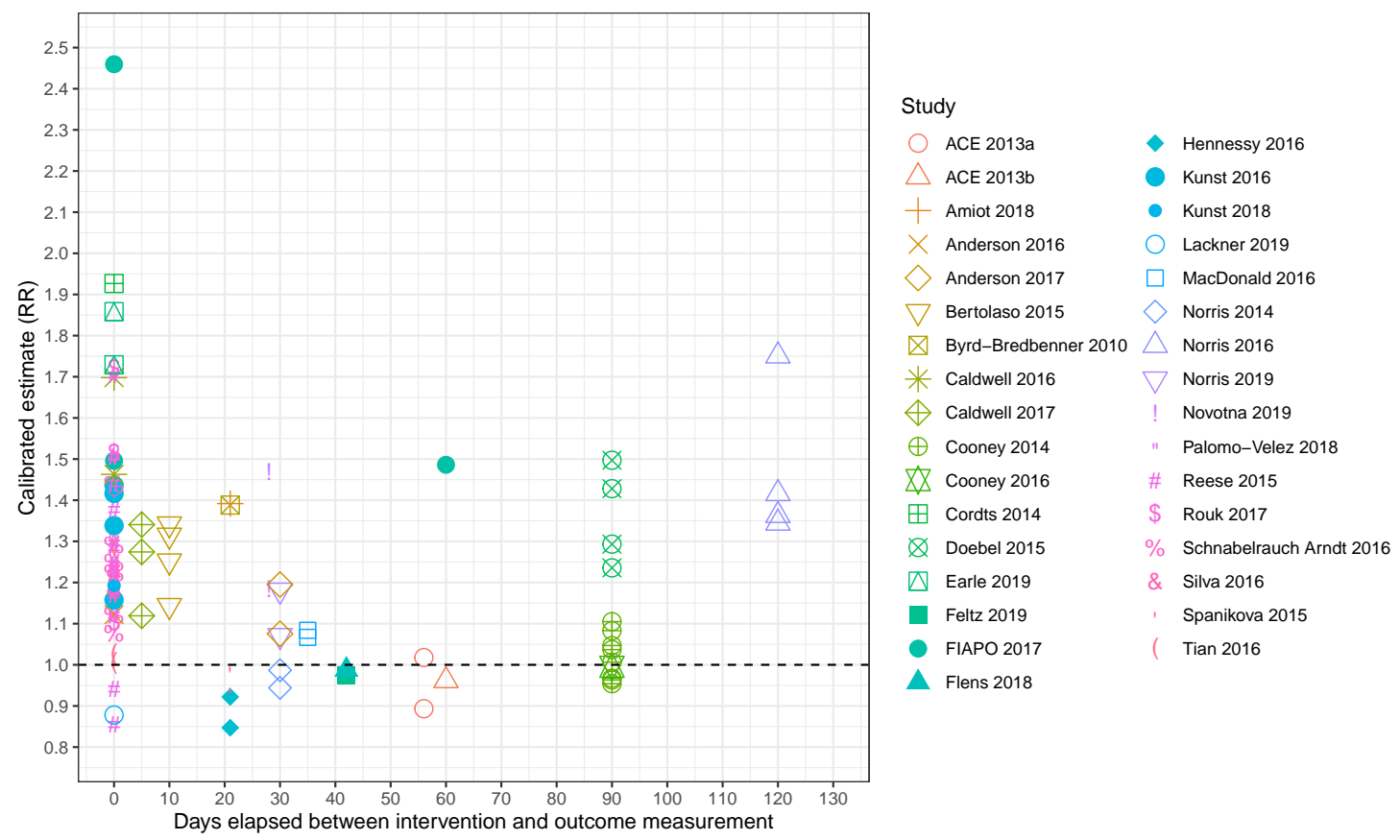

Figure S5: Time elapsed between intervention and outcome measurement versus calibrated estimate of true population effect size. Horizontal dashed line: null.

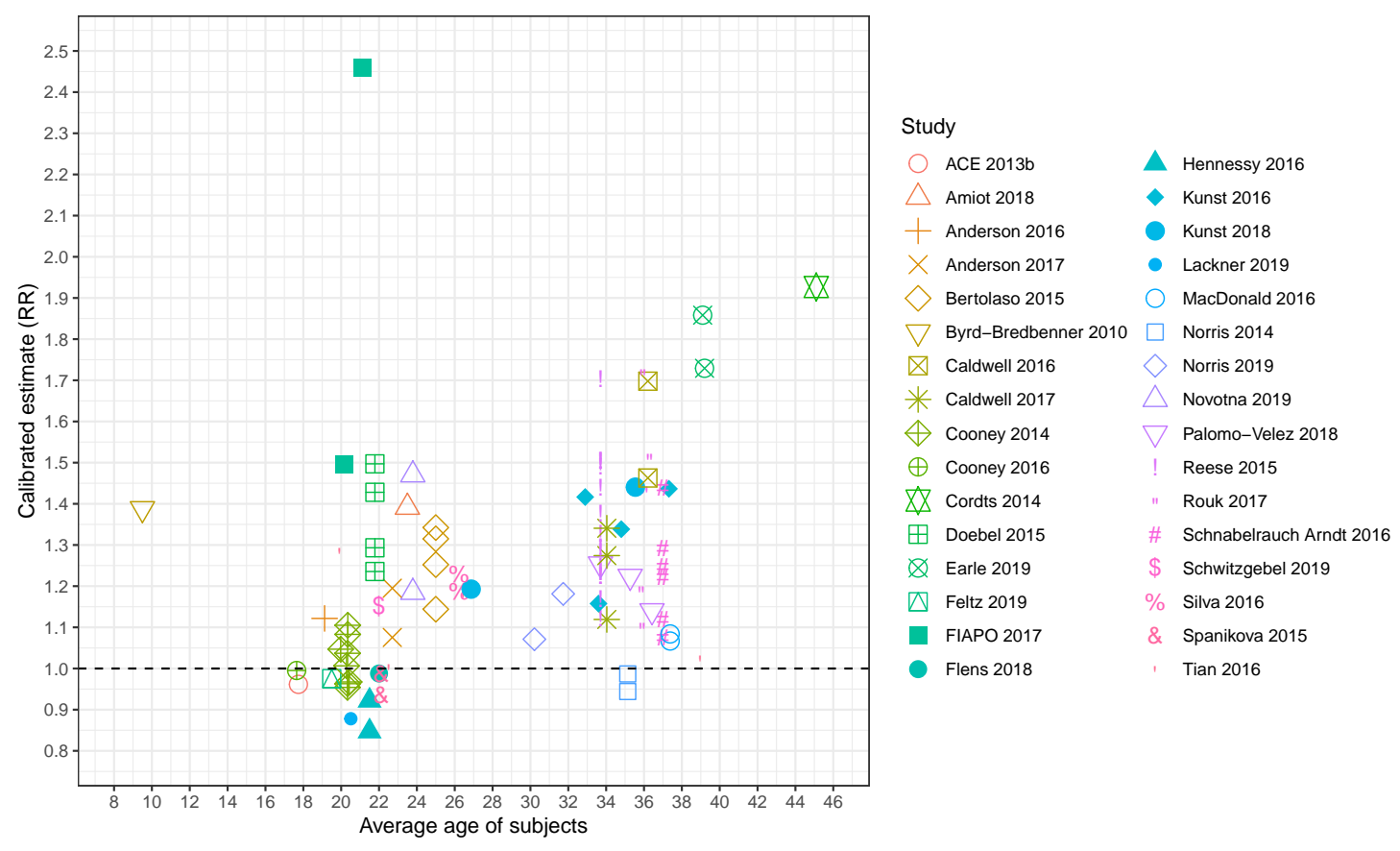

Figure S6: Average subject age versus calibrated estimate of true population effect size. Horizontal dashed line: null. 


\section{Changes AND DEVIATIONS FROM PREREGistered PROTOCOL}

Here, we describe the three stages in which we preregistered the protocol and describe changes and deviations from the protocol at each stage. After conducting initial searches, but before extracting data, we first registered the protocol on July 3, 2019. During data extraction, we updated the protocol to: (1) describe new plans to compare our results to those of a previous qualitative review ${ }^{5}$; (2) change the way that we would handle multiple outcomes per study (from always choosing the broadest-scoped outcome to choosing the outcome most closely matching the intervention's scope, a change affecting only a few studies); and (3) we removed caveats about omitting certain analyses if estimates were non-normal in light of new statistical methods obviating this assumption $\frac{19}{}$. With these changes, we registered the protocol again on July 16, 2019 and submitted this version to the journal Systematic Reviews. In response to peer review comments, we made further revisions to remove the comparison to the previous review. Ultimate analyses deviated from the published protocol paper ${ }^{[18}$ in four ways: (1) we extracted risk ratios rather than standardized mean differences because studies very often used pseudo-continuous, non-normal Likert outcomes, jeopardizing the validity of the needed effect-size conversions to the standardized mean difference scale; (2) we omitted the animal-centric, health-centric, environment-centric metrics; (3) we omitted sensitivity analyses that would have excluded interventions targeting consumption of only specific types of meat; and (4) we omitted self-selected within-subjects studies from main analyses. More details and reasons for these decisions are given in the main text. We also conducted several post hoc analyses, all of which are described as such in the main text.

\section{REFERENCES}

[1] Effective Thesis. http://effectivethesis.com/.

[2] Symposium on Multidisciplinary Research in Effective Animal Advocacy. https: //animalcharityevaluators.org/about/events/2016-symposium/, 2016.

[3] Psychology of sustainable consumption. http://www.psychologyof sustainableconsu mption.com/people--abstracts.html, 2018.

[4] Animal Aid. $57 \%$ of Summer Vegan Pledge survey respondents to remain vegan. Technical report, Animal Abuse Injustice and Defence Society, Aug. 2019. https://www.animalaid.org.uk/57-of-summer-vegan-pledge-survey-respondents-toremain-vegan/.

[5] F. Bianchi, C. Dorsel, E. Garnett, P. Aveyard, and S. A. Jebb. Interventions targeting conscious determinants of human behaviour to reduce the demand for meat: A systematic review with qualitative comparative analysis. International Journal of Behavioral Nutrition and Physical Activity, 15(1):102, 2018. 
[6] G. Boese, G. Stuessy, K. Asher, S. Hennessy, B. Macdonald, H. Sethu, and P. Moleman. Animal advocacy data repository, July 2016.

[7] T. Bradbury. Over half of Summer Vegan Pledge participants to remain vegan. Technical report, Animal Aid, Aug. 2018. https://www.animalaid.org.uk/wpcontent/uploads/2018/08/SVP-Survey-PR-PDF.pdf.

[8] J. Cohen. A coefficient of agreement for nominal scales. Educational and Psychological Measurement, 20(1):37-46, 1960.

[9] N. Cooney. Veganomics. Lantern Books, 2014.

[10] G. S. de Lanauze and B. Siadou-Martin. Dissonant cognitions: From psychological discomfort to motivation to change. Journal of Consumer Marketing, 2019.

[11] E. Dowsett, C. Semmler, H. Bray, R. A. Ankeny, and A. Chur-Hansen. Neutralising the meat paradox: Cognitive dissonance, gender, and eating animals. Appetite, 123:280-288, 2018.

[12] M. Earle, G. Hodson, K. Dhont, and C. MacInnis. Eating with our eyes (closed): Effects of visually associating animals with meat on antivegan/vegetarian attitudes and meat consumption willingness. Group Processes and Intergroup Relations, 22(6):1-18, 2019.

[13] A. C. Evaluators. Leafleting. Technical report, nov 2017.

[14] V. Hasselblad and L. V. Hedges. Meta-analysis of screening and diagnostic tests. Psychological Bulletin, 117(1):167, 1995.

[15] J. P. Higgins, D. G. Altman, P. C. Gøtzsche, P. Jüni, D. Moher, A. D. Oxman, J. Savović, K. F. Schulz, L. Weeks, and J. A. Sterne. The Cochrane Collaboration's tool for assessing risk of bias in randomised trials. BMJ, 343:d5928, 2011.

[16] P. Hurford and M. Davis. Animal Equality showed that advocating for diet change works. But is it cost-effective? https://www.rethinkpriorities.org/blog/2019/2/ 21/animal-equality-showed-that-advocating-for-diet-change-works-but-is -it-cost-effective-1, June 2018.

[17] P. Jacobs and W. Viechtbauer. Estimation of the biserial correlation and its sampling variance for use in meta-analysis. Research synthesis methods, 8(2):161-180, 2017.

[18] M. B. Mathur, T. N. Robinson, D. B. Reichling, C. D. Gardner, J. Nadler, P. A. Bain, and J. Peacock. Reducing meat consumption by appealing to animal welfare: Protocol for a meta-analysis and theoretical review. Systematic Reviews, 9(1):1-8, 2020.

[19] M. B. Mathur and T. J. VanderWeele. Robust metrics and sensitivity analyses for meta-analyses of heterogeneous effects. Epidemiology, 9(1):1-8, 2020. https://doi.org/10.1097/ede.0000000000001180. 
[20] L.-A. McNutt, C. Wu, X. Xue, and J. P. Hafner. Estimating the relative risk in cohort studies and clinical trials of common outcomes. American Journal of Epidemiology, 157(10):940-943, 2003.

[21] D. Miceli. Veganuary 2018: The results are in! Technical report, Veganuary, 2018. https://uk.veganuary.com/blog/veganuary-2018-the-results-are-in/.

[22] T. VanderWeele. On a square-root transformation of the odds ratio for a common outcome. Epidemiology (Cambridge, Mass.), 28(6):e58, 2017.

[23] T. J. VanderWeele and Y. Li. Simple sensitivity analysis for differential measurement error. American Journal of Epidemiology, 188(10):1823-1829, 2019.

[24] Veganuary. Veganuary 2017 survey results. Technical report, 2017. https://uk.veganuary.com/blog/veganuary-2017-survey-results/.

[25] Veganuary. Veganuary 2019: The results are in! Technical report, 2019. https://uk.veganuary.com/blog/veganuary-2019-the-results-are-in/.

[26] W. Viechtbauer et al. Conducting meta-analyses in $\mathrm{R}$ with the metafor package. Journal of Statistical Software, 36(3):1-48, 2010.

[27] H. White et al. A heteroskedasticity-consistent covariance matrix estimator and a direct test for heteroskedasticity. Econometrica, 48(4):817-838, 1980.

[28] G. Y. Zou. One relative risk versus two odds ratios: implications for meta-analyses involving paired and unpaired binary data. Clinical Trials, 4(1):25-31, 2007. 